\title{
Data from Theodolite Measurements of Creep Rates on San Francisco Bay Region Faults, California: 1979-2001
}

By Jon S. Galehouse ${ }^{1}$

Open-File Report 02-225

2002

This report is preliminary and has not been reviewed for conformity with U.S. Geological Survey editorial standards or with the North American Stratigraphic Code. Any use of trade, firm, or product names is for descriptive purposes only and does not imply endorsement by the U.S. Government.

U.S. DEPARTMENT OF THE INTERIOR

U.S. GEOLOGICAL SURVEY

${ }^{1}$ Department of Geosciences, San Francisco State University, San Francisco, CA 94132. Present address: P.O. Box 140, Twain, CA 95984. 


\section{INTRODUCTION}

My purpose is to make our creep data on San Francisco Bay region active faults available to the scientific research community. My student research assistants and I measured creep (aseismic slip) rates on these faults from 1979 until my retirement from the project in 2001 . These data are further described in my final technical report as principal investigator, which summarizes results from 22 September 1979 through 28 February 2001 (Galehouse, 2001). We made over 2600 creep measurements, about one-third in the ten years prior to the Loma Prieta earthquake (LPEQ) and twothirds in the 11.4 years following it. The measurements are continuing to be made by members of the Geosciences Department at San Francisco State University (SFSU) under the direction of Karen Grove and John Caskey. A complete analysis of our results obtained on the Hayward fault is presented in Lienkaemper, Galehouse and Simpson (2001). A formal report based on the entire San Francisco Bay region data set is in preparation.

\section{METHODS}

Amount of slip was determined by noting changes in angles between sets of measurements taken across a fault at different times. This triangulation method uses a theodolite to measure the angle formed by three fixed points to the nearest tenth of a second of arc (Galehouse and others, 1982). For the first 14 years of measurements, the angle was measured 12 times each measurement day. Since then, we have been measuring it eight times each day. The amount of slip between measurement days can be calculated trigonometrically using the change in average angle. The precision of the measurement method is such that we can detect with confidence any movement more than 1-2 mm between successive measurement days.

We have regular measurement sites at 29 localities on active faults, plus data from five sites that had to be abandoned. These site locations are shown as triangles and site numbers on the accompanying map (Figure 1) and identified by name in Tables 1 and 2A and in the data sheets. In addition to the sites in the San Francisco Bay region, we have one measurement site on the San Andreas fault in the Point Arena area and two on the Maacama fault in Willits and east of Ukiah. We typically have measured most sites with a history of creep about once every two months and most sites without any creep history about every three months.

In addition to our eleven regular sites on the Hayward fault, we have established 20 additional sites (shown by dots in Figure 1 and by name in Table 2B and in the data sheets) with J. Lienkaemper of the USGS. We began measuring each of these additional sites annually in July August 1994.

\section{DA TA}

Table 1 shows the least squares average rate of movement at each site determined using linear regression and the simple average determined by dividing the total net right-lateral displacement by the total time measured. All measurement sites span a fault width of 57-267 m, except Sites 8 and 20 that span a greater width because of site considerations. This fault width spanned is noted in Table 2 and represents the distance from the theodolite on one side of a fault (IS) to a target on the other side (ES). Table 2 also shows any changes in lengths during measurement history. Data sheets for each site follow Table 2. Each data sheet is identified in the upper left by site number and name and Hayward fault sites are ordered from northwest to southeast using kilometer distances along the fault measured from Point Pinole (P, in Figure 1) using the grid in Lienkaemper (1992). These data are also available for downloading in the Excel format to facilitate analysis of the data at http://geopubs.wr.usgs.gov/open-file/of02-225/ (SFBayRegion.xls and HaywardFault.xls). They are also available as tab delimited raw data. The data include all regular measurement sites, SF-1 through SF-34 and the 20 SFSU and USGS afterslip sites on the Hayward fault. 


\section{ACKNOWLEDGMENTS}

This project has been continuously funded since 1979 by various grants and contracts from the U.S. Geological Survey, National Earthquake Hazards Reduction Program (latest contract was 99-HQ-GR-0084). Special thanks go to the 23 student research assistants from San Francisco State University who have been instrumental in collecting these theodolite data since 1979. I am particularly grateful to Brett Baker, Beth Brown, Carolyn Garrison, Oliver Graves, Theresa Hoyt, Forrest McFarland, Carl Schaefer, and Jim Thordsen who each worked with me for more than three years. Thanks also to Bob Abrams, Chris Alger, Linda Bond, Denise Coutlakis, Carolyn Domrose, Matt Harrigan, CJ Hayden, Kathleen Isaacson, Dan McVanner, Barbara Menne, Nicole Peirce, Brian Pierce, Holly Prochaska, Debra Smith, and Leta Smith who have all served as theodolite operators. Special thanks to James Lienkaemper of the U.S. Geological Survey for his help and encouragement over the years, including suggestions and thoughtful reviews of this manuscript. Reviews by R.W. Simpson and W. D. Stuart also improved the clarity of the map and text.

\section{REFERENCES CITED}

Galehouse, J.S., Brown, B. D., Pierce, B., and Thordsen J. J., 1982, Changes in movement rates on certain East Bay faults, in California Division of Mines and Geology Special Publication 62, 239-250.

Galehouse, J.S., 2001, Theodolite measurements of creep rates on San Francisco Bay Region faults, National Earthquake Hazards Reduction Program, Annual Project Summaries, Volume 42, U. S. Geological Survey, Reston, VA. (on line report) http://erp-web.er.usgs.gov/reports/annsum/vol42/nc/G0084.htm

Lienkaemper, J.J., 1992, Map of recently active traces of the Hayward Fault, Alameda and Contra Costa Counties, California: U.S. Geological Survey Miscellaneous Field Studies Map MF-2196, 13 p. (1 sheet).

Lienkaemper, J.J., Galehouse, J.S., and Simpson, R.W., 2001, Long-term monitoring of creep rate along the Hayward fault and evidence for a lasting creep response to 1989 Loma Prieta earthquake: Geophysical Research Letters, v. 28, $2265-$ 2268.

Working Group on California Earthquake Probabilities (WG99), Earthquake probabilities in the San Francisco Bay region: 2000 to 2030-A summary of findings, U. S. Geological Survey Open-File Rep., 99-517. (on line report) http://geopubs.wr.usgs.gov/open-file/of99-517 


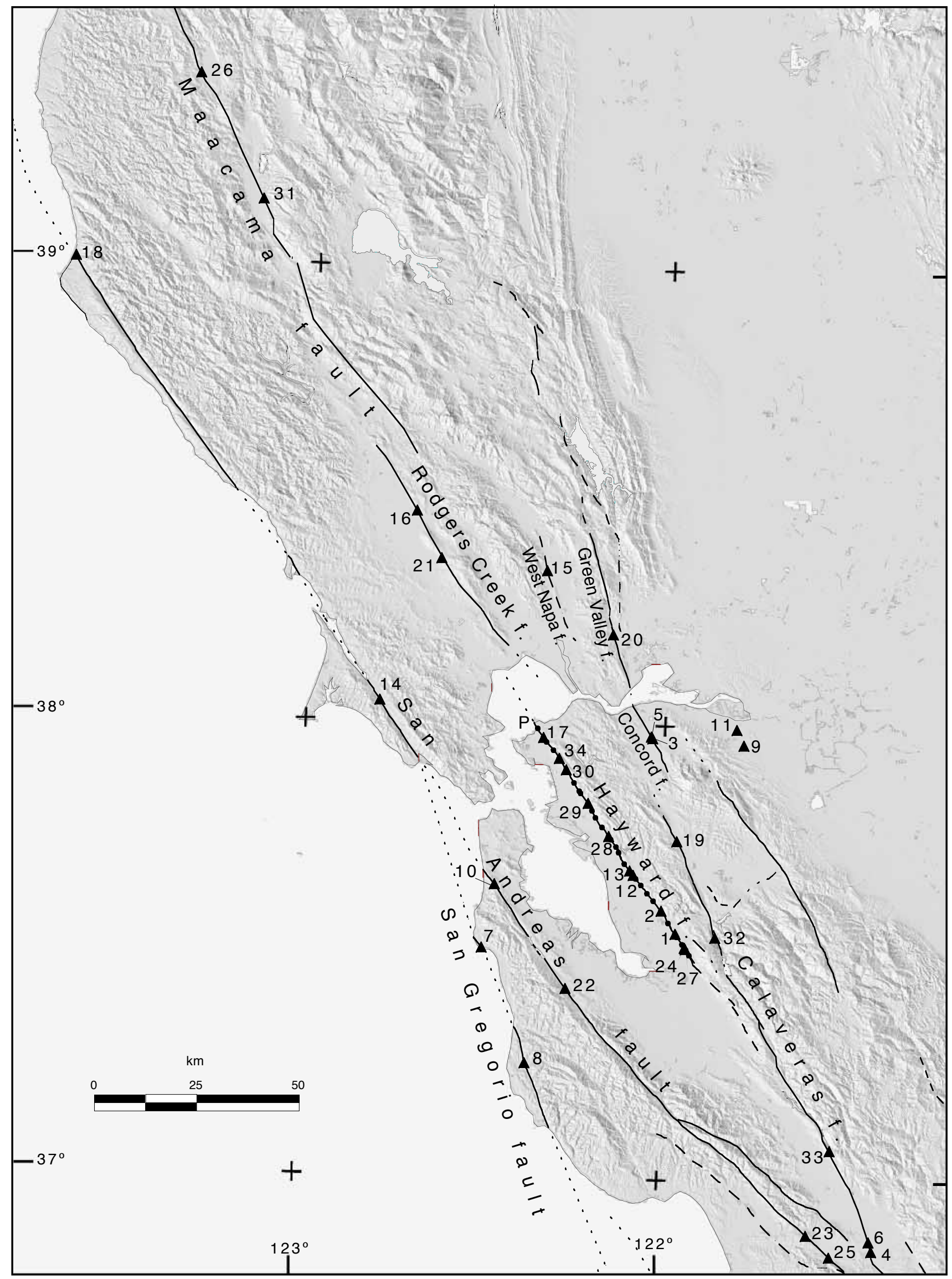

Figure 1. Locations of alinement arrays. Regular arrays shown as triangles, afterslip arrays on Hayward Fault shown as dots. P, Point Pinole. 
Table 1. Average Rates of Right-Lateral Movement

\begin{tabular}{|c|c|c|c|c|c|c|c|c|c|}
\hline \multirow[b]{2}{*}{ Fault } & \multirow[b]{2}{*}{$\begin{array}{l}\text { WG99 } \\
\text { Segment } \\
\text { Code }\end{array}$} & \multirow[b]{2}{*}{ Site \# } & \multirow[b]{2}{*}{ Site } & \multirow[b]{2}{*}{$\begin{array}{c}\text { Least } \\
\text { Squares } \\
\text { Average } \\
(\mathrm{mm} / \mathrm{yr})\end{array}$} & \multirow[b]{2}{*}{$\begin{array}{l}\text { Simple } † \\
\text { Average } \\
(\mathrm{mm} / \mathrm{yr})\end{array}$} & \multirow[b]{2}{*}{$\begin{array}{c}\text { Years } \\
\text { Measrd }\end{array}$} & \multicolumn{3}{|c|}{ Pre-LPEQ*: } \\
\hline & & & & & & & $\begin{array}{c}\text { Least } \\
\text { Squares } \\
\text { Average } \\
(\mathrm{mm} / \mathrm{yr})\end{array}$ & $\begin{array}{l}\text { Simple } † \\
\text { Average } \\
(\mathrm{mm} / \mathrm{yr})\end{array}$ & $\begin{array}{c}\text { Years } \\
\text { Measrd }\end{array}$ \\
\hline \multirow{2}{*}{$\begin{array}{c}\text { San Andreas } \\
"\end{array}$} & NCS & 18 & Alder Creek & 0.5 & 0.6 & 19.6 & 0.9 & 0.9 & 8.0 \\
\hline & NCS & 14 & Point Reyes & 0.2 & 0.1 & 15.9 & 0.4 & 0.4 & 4.7 \\
\hline$"$ & $\mathrm{PN}$ & 10 & Duhallow Way & -0.3 & -0.2 & 20.8 & -0.3 & -0.2 & 9.5 \\
\hline$"$ & PN & 22 & Roberta Drive & 0.3 & 0.7 & 11.1 & - & 0.0 & 12.7 \\
\hline$"$ & SCZ & 23 & Cannon Road & 0.1 & 0.2 & 8.2 & - & & \\
\hline$"$ & SCZ & 25 & Mission Vineyard Rd & 10.4 & 11.2 & 10.6 & - & 6.7 & $\sim 20$ \\
\hline \multirow{2}{*}{$\begin{array}{l}\text { Hayward } \\
\text { " }\end{array}$} & $\mathrm{NH}$ & 17 & Contra Costa College & 4.9 & 4.6 & 20.4 & 4.7 & 4.2 & 9.0 \\
\hline & NH & 34 & Thors Bay Road & 4.3 & 4.5 & 11.3 & - & & \\
\hline$"$ & $\mathrm{NH}$ & 30 & Florida Ave & 1.4 & 1.5 & 8.3 & - & & \\
\hline " & $\mathrm{NH}$ & 29 & LaSalle Ave & 3.8 & 4.2 & 7.9 & - & & \\
\hline$"$ & $\mathrm{SH}$ & 28 & Encina Way & 3.0 & 3.8 & 11.4 & - & & \\
\hline$"$ & $\mathrm{SH}$ & 13 & Rose Street & 5.0 & 4.8 & 20.5 & 4.9 & 4.6 & 9.3 \\
\hline$"$ & $\mathrm{SH}$ & 12 & D Street & 4.4 & 4.5 & 20.5 & 4.9 & 4.9 & 9.3 \\
\hline$"$ & $\mathrm{SH}$ & 2 & Appian Way & 5.2 & 5.4 & 21.3 & 4.7 & 4.6 & 9.9 \\
\hline$"$ & $\mathrm{SH}$ & 1 & Rockett Drive & 4.8 & 5.0 & 21.3 & 5.5 & 4.5 & 9.9 \\
\hline$"$ & $\mathrm{SH}$ & 24 & Camellia Drive & 3.9 & 3.2 & 11.0 & - & 9.5 & $\sim 20$ \\
\hline$"$ & $\mathrm{SH}$ & 27 & Parkmeadow Drive & 5.7 & 4.8 & 8.9 & - & 8.2 & $\sim 14$ \\
\hline \multirow{2}{*}{$\begin{array}{c}\text { Rodgers Creek } \\
" ~\end{array}$} & $\mathrm{RC}$ & 16 & Nielson Road & 0.4 & -0.9 & 5.4 & - & & \\
\hline & $\mathrm{RC}$ & 21 & Roberts Road & 1.6 & 1.0 & 14.2 & - & & \\
\hline \multirow{2}{*}{$\begin{array}{c}\text { Calaveras } \\
"\end{array}$} & $\mathrm{NC}$ & 19 & Corey Place & 1.6 & $1.7(3.9)$ & $20.2(8.3)$ & 0.2 & 0.3 & 8.6 \\
\hline & $\mathrm{NC}$ & 32 & Welch Creek Road & 3.6 & 3.5 & 4.0 & - & & \\
\hline$"$ & $\mathrm{CC}$ & 33 & Coyote Ranch & 13.9 & 14.3 & 4.0 & 16.6 & 17.2 & 20.0 \\
\hline$"$ & $\mathrm{SC}$ & 6 & Wright Road & 9.0 & 9.9 & 21.3 & 12.2 & 12.1 & 9.8 \\
\hline$"$ & $\mathrm{SC}$ & 4 & Seventh Street & 6.4 & 6.6 & 21.4 & 6.4 & 6.8 & 9.9 \\
\hline Green Valley & SGV & 20 & Red Top Road & 4.2 & 4.2 & 16.7 & 4.3 & 4.2 & 5.1 \\
\hline \multirow{2}{*}{$\begin{array}{c}\text { Concord } \\
"\end{array}$} & $\mathrm{CON}$ & 5 & Salvio Street & 2.7 & 2.8 & 21.3 & 2.5 & 3.2 & 9.9 \\
\hline & $\mathrm{CON}$ & 3 & Ashbury Drive & 3.6 & 3.8 & 21.3 & 3.3 & 3.8 & 9.9 \\
\hline \multirow{2}{*}{$\begin{array}{l}\text { Maacama } \\
\text { " }\end{array}$} & - & 26 & W. Commercial Ave & 6.5 & 6.6 & 9.3 & - & & \\
\hline & - & 31 & Sanford Ranch Road & 4.4 & 4.5 & 7.8 & 一 & & \\
\hline Seal Cove & SGN & 7 & West Point Ave & -0.1 & -0.1 & 21.0 & 0.2 & 0.4 & 9.7 \\
\hline San Gregorio & SGN & 8 & Pescadero Road & 0.8 & 0.4 & 18.5 & -0.8 & -0.9 & 7.2 \\
\hline West Napa & - & 15 & Linda Vista Ave & 0.1 & 0.0 & 18.5 & - & & \\
\hline "Antioch" & - & 11 & Worrell Road & -0.1 & -0.3 & 19.8 & - & & \\
\hline$"$ & -- & 9 & Deer Valley Road & 1.7 & 1.2 & 7.6 & - & & \\
\hline
\end{tabular}

*LPEQ - M7, Loma Prieta earthquake, October 17, 1989

$\dagger$ Simple Average, (cumulative movement)/(years measured) 
Table 2. IS to ES Distance* History, SFSU Measurements, 22 Sep. 1979 to 28 Feb. 2001

\section{A. Regular SFSU Sites}

\begin{tabular}{|c|c|c|c|c|c|}
\hline Site \# & Site Name & $\begin{array}{l}\text { IS-ES Distance } \\
\text { (m) (Active† } \\
\text { underlined) }\end{array}$ & $\begin{array}{c}\text { First } \\
\text { Measurement }\end{array}$ & $\begin{array}{c}\text { Last } \\
\text { Measurement }\end{array}$ & Abandoned \\
\hline 1 & Rockett Drive & $\underline{180.0}$ & 22-Sep-79 & 30 -Dec-00 & \\
\hline \multirow[t]{3}{*}{2} & Appian Way & $\overline{145.6}$ & 23-Sep-79 & 9-Jun-81 & \\
\hline & & 148.3 & 6-Aug-81 & 15-Jan-93 & \\
\hline & & 125.2 & 15-Jan-93 & 30-Dec-00 & \\
\hline \multirow[t]{2}{*}{3} & Ashbury Drive & 143.0 & 28 -Sep-79 & $6-O c t-85$ & \\
\hline & & 130.0 & 23-Mar-86 & $11-F e b-01$ & \\
\hline 4 & Seventh Street & 89.7 & 29-Sep-79 & $25-$ Feb-01 & \\
\hline 5 & Salvio Street & 57.1 & 30-Sep-79 & $11-F e b-01$ & \\
\hline \multirow[t]{2}{*}{6} & Wright Road & $\overline{51.7}$ & 21-Oct-79 & $11-F e b-95$ & \\
\hline & & 103.4 & 12-Nov-94 & $25-F e b-01$ & \\
\hline \multirow[t]{3}{*}{7} & West Point Ave & 176.9 & 9-Nov-79 & 2-Apr-81 & \\
\hline & & 167.7 & 8-Jul-81 & 27-Jan-90 & \\
\hline & & 266.6 & 25-Mar-90 & 18 -Nov-00 & \\
\hline \multirow[t]{2}{*}{8} & Pescadero Road & 452.0 & 20-May-82 & 6-Feb-93 & \\
\hline & & 455.0 & 12-Sep-93 & 18 -Nov-00 & \\
\hline 9 & Deer Valley Road & 226.2 & 21-Nov-82 & 1-Jul-90 & $\mathrm{X}$ \\
\hline \multirow[t]{3}{*}{10} & Duhallow Way & 173.9 & 23-Mar-80 & $3-N o v-89$ & \\
\hline & & 207.5 & $15-$ Nov-89 & 29-May-94 & \\
\hline & & 205.8 & 6-Aug-94 & 13-Jan-01 & \\
\hline \multirow[t]{2}{*}{11} & Worrell Road & 103.9 & 4-May-80 & 15-Mar-97 & \\
\hline & & 103.8 & 5-Jul-97 & $27-F e b-00$ & $\mathrm{X}$ \\
\hline \multirow[t]{2}{*}{12} & D Street & 136.2 & 23-Jun-80 & 13-Jul-97 & \\
\hline & & 112.4 & 12-Jun-99 & 6-Jan-01 & \\
\hline \multirow[t]{2}{*}{13} & Rose Street & $\overline{84.5}$ & 24-Jun-80 & 11-Jul-92 & \\
\hline & & $\underline{153.9}$ & 24-Sep-92 & 6-Jan-01 & \\
\hline 14 & Point Reyes & 70.6 & $4-F e b-85$ & 13-Jan-01 & \\
\hline \multirow[t]{4}{*}{15} & Linda Vista Ave & $\overline{128.0}$ & 26-Jul-80 & 15-Aug-81 & \\
\hline & & 131.6 & 9-Nov-81 & 21-Jul-84 & \\
\hline & & 131.0 & 9-Dec-84 & 10-Jul-93 & \\
\hline & & 130.9 & 25-Sep-93 & 16-Jan-99 & $\mathrm{X}$ \\
\hline 16 & Nielson Road & 209.1 & 17-Aug-80 & 20-Jan-86 & $\mathrm{X}$ \\
\hline \multirow[t]{2}{*}{17} & Contra Costa College & 75.7 & 10 -Aug-80 & 4-Mar-90 & \\
\hline & & 106.8 & 4-Mar-90 & 21-Jan-01 & \\
\hline 18 & Alder Creek & 267.4 & 9-Jan-81 & 5-Aug-00 & \\
\hline 19 & Corey Place & $\overline{111.1}$ & $23-N o v-80$ & $10-$ Feb-01 & \\
\hline \multirow[t]{3}{*}{20} & Red Top Road & $\overline{335.8}$ & 15-Jun-84 & 28-Feb-99 & \\
\hline & & 352.9 & 12-Mar-00 & 15 -Oct-00 & \\
\hline & & $\underline{355.4}$ & 10-Dec-00 & $11-$ Feb-01 & \\
\hline \multirow[t]{2}{*}{21} & Roberts Road & $\overline{199.6}$ & 20-Sep-86 & 23-Jan-93 & \\
\hline & & 198.7 & 17-Apr-93 & 26-Nov-00 & \\
\hline 22 & Roberta Drive & $\overline{91.2}$ & 4-Nov-89 & 26-Nov-00 & \\
\hline \multirow[t]{2}{*}{23} & Cannon Road & $\overline{88.0}$ & 18-Nov-89 & 13-Apr-96 & \\
\hline & & 88.1 & 20-Jul-96 & 14-Feb-98 & $\mathrm{X}$ \\
\hline 24 & Camellia Drive & $\underline{88.6}$ & $11-$ Feb-90 & $10-$ Feb-01 & \\
\hline 25 & Mission Vineyard Rd & $\overline{135.0}$ & 21-Jul-90 & 25-Apr-91 & \\
\hline
\end{tabular}




\begin{tabular}{|c|c|c|c|c|}
\hline & & 134.2 & 13-Jul-91 & $25-F e b-01$ \\
\hline \multirow[t]{2}{*}{26} & W. Commercial Ave & 105.1 & 14-Nov-91 & 8-May-94 \\
\hline & & $\underline{126.1}$ & 9-Jul-94 & $24-F e b-01$ \\
\hline 27 & Parkmeadow Drive & $\overline{157.4}$ & 5-Apr-92 & 10 -Feb-01 \\
\hline \multirow[t]{2}{*}{28} & Encina Way & $\overline{105.4}$ & 10-Sep-89 & 23-Dec-95 \\
\hline & & $\underline{122.3}$ & 6-Apr-96 & 20-Jan-01 \\
\hline \multirow[t]{2}{*}{29} & LaSalle Ave & 182.5 & $10-F e b-93$ & 16-Jan-00 \\
\hline & & $\underline{183.2}$ & 9-Jul-00 & 20-Jan-01 \\
\hline \multirow[t]{3}{*}{30} & Florida Ave & 78.8 & 12-Aug-92 & $10-F e b-93$ \\
\hline & & 73.6 & $10-F e b-93$ & 27-Apr-97 \\
\hline & & 125.3 & 16-Aug-97 & 12-Nov-00 \\
\hline \multirow[t]{2}{*}{31} & Sanford Ranch Road & 263.2 & 22-May-93 & $10-$-Oct-99 \\
\hline & & $\underline{262.3}$ & 11-Dec-99 & 24-Feb-01 \\
\hline 32 & Welch Creek Road & 164.1 & 24-Jan-97 & 27-Jan-01 \\
\hline 33 & Coyote Ranch & $\underline{99.2}$ & 3-May-68 & 27-Jan-01 \\
\hline 34 & Thors Bay Road & 120.0 & 30-Sep-89 & 21-Jan-01 \\
\hline
\end{tabular}

B. SFSU-USGS Afterslip Arrays on Hayward Fault, Measured Annually

\begin{tabular}{|c|c|c|c|c|}
\hline $\begin{array}{l}\text { Distance from } \\
\text { Pt. Pinole } \\
(\mathrm{km})\end{array}$ & Site Name & $\begin{array}{l}\text { IS-ES Distance } \\
\text { (m) (Active } \dagger \\
\text { underlined) }\end{array}$ & $\begin{array}{c}\text { First } \\
\text { measurement }\end{array}$ & $\begin{array}{c}\text { Last } \\
\text { measurement }\end{array}$ \\
\hline 8.37 & Olive & 149.0 & 30-Sep-89 & 12-Nov-00 \\
\hline 17.82 & Cal Stadium & $\underline{165.5}$ & 27-Jun-93 & 29-Oct-00 \\
\hline \multirow[t]{2}{*}{20.84} & Temescal & $\overline{148.0}$ & 12-Feb-94 & 22-Aug-95 \\
\hline & & 153.9 & 27-Sep-98 & $12-$ Nov-00 \\
\hline \multirow[t]{2}{*}{25.98} & Lincoln & $\overline{92.8}$ & 1-Aug-94 & 7-Sep-98 \\
\hline & & 110.4 & 11-Sep-99 & $12-$ Nov-00 \\
\hline 27.81 & 39th & $\underline{131.9}$ & 13-Aug-92 & 11-Nov-00 \\
\hline 30.68 & 73rd & 89.8 & 22-May-93 & 28 -Oct-00 \\
\hline 36.55 & Chabot Park & $\overline{231.2}$ & 22-May-93 & 28 -Oct- 00 \\
\hline 38.28 & Fairmont & 166.6 & 17-Aug-97 & 28 -Oct- 00 \\
\hline 41.11 & 167 th & 90.9 & 14-Aug-92 & 28 -Oct- 00 \\
\hline \multirow[t]{2}{*}{45.64} & Palisade & $\overline{102.8}$ & 11-Oct-89 & 20-Aug-96 \\
\hline & & 177.5 & 22-Oct-92 & 21 -Oct-00 \\
\hline \multirow[t]{2}{*}{47.72} & Sepulchre & 87.9 & 3-Aug-94 & 19-Aug-96 \\
\hline & & 106.9 & 18-Aug-97 & $21-$ Oct-00 \\
\hline 50.15 & Woodland & 72.7 & $12-N o v-92$ & $21-$ Oct-00 \\
\hline 52.60 & Chimes & $\overline{120.3}$ & 4-Aug-94 & $11-$ Nov-00 \\
\hline 59.09 & Gilbert & $\overline{89.3}$ & 19-Nov-92 & 4-Nov-00 \\
\hline 62.64 & Hancock & $\overline{88.5}$ & 17-Aug-94 & 4-Nov-00 \\
\hline 63.10 & Union & $\overline{168.1}$ & 7-Jan-93 & 4-Nov-00 \\
\hline 65.29 & Pine & $\overline{98.0}$ & 8-Jan-93 & $11-$ Nov-00 \\
\hline 67.02 & S. Grimmer & $\overline{129.5}$ & 9-Jun-93 & $11-$ Nov-00 \\
\hline 67.21 & Onondaga & $\overline{72.9}$ & 17-Aug-94 & $11-$ Nov-00 \\
\hline 68.45 & Mission & $\overline{168.9}$ & 10-Mar-93 & $11-$ Nov-00 \\
\hline
\end{tabular}

*IS-ES distance, length of fault crossing line from instrument station (IS) to target on end station (ES).

$\dagger$ Active, i.e., the line currently being monitored 
SF-01 Rockett Drive in Fremont Hayward Fault 62.25 km

\begin{tabular}{|c|c|c|c|c|c|c|c|c|}
\hline $\begin{array}{l}\text { movement } \\
(\mathrm{mm})\end{array}$ & std. dev. & \# of years & year & date & $\begin{array}{c}\text { simple } \\
\text { average }\end{array}$ & $\begin{array}{l}\text { average } \\
(\mathrm{mm} / \mathrm{yr})\end{array}$ & $\begin{array}{l}\text { average } \\
(\mathrm{mm} / \mathrm{yr})\end{array}$ & $\begin{array}{l}\text { average } \\
(\mathrm{mm} / \mathrm{yr})\end{array}$ \\
\hline 0.0 & 2.9 & 0.000 & 1979.726 & 22-Sep-79 & & & & \\
\hline-7.0 & 1.3 & 0.192 & 1979.918 & 1-Dec-79 & & & & \\
\hline-3.2 & 4.5 & 0.383 & 1980.109 & 9-Feb-80 & & & & \\
\hline 1.1 & 4.3 & 0.498 & 1980.224 & 22-Mar-80 & & & & \\
\hline 3.7 & 1.4 & 0.777 & 1980.503 & 2-Jul-80 & & & & \\
\hline 3.7 & 1.4 & 0.982 & 1980.708 & $15-$ Sep-80 & 3.8 & 8.0 & & \\
\hline 3.4 & 2.9 & 1.072 & 1980.798 & 18-Oct-80 & 3.2 & 7.4 & & \\
\hline 2.5 & 2.4 & 1.269 & 1980.995 & 29-Dec-80 & 2.0 & 6.1 & & \\
\hline 4.5 & 1.5 & 1.403 & 1981.129 & 16-Feb-81 & 3.2 & 5.9 & & \\
\hline 5.0 & 1.6 & 1.455 & 1981.181 & 7-Mar-81 & 3.4 & 5.8 & & \\
\hline 6.8 & 1.6 & 1.712 & 1981.438 & 9-Jun-81 & 4.0 & 5.8 & & \\
\hline 4.4 & 2.0 & 1.858 & 1981.584 & 1-Aug-81 & 2.4 & 5.1 & & \\
\hline 6.2 & 1.3 & 2.203 & 1981.929 & 5-Dec-81 & 2.8 & 4.7 & & \\
\hline 7.3 & 0.9 & 2.356 & 1982.082 & 30-Jan-82 & 3.1 & 4.5 & & \\
\hline 8.3 & 3.4 & 2.471 & 1982.197 & 13-Mar-82 & 3.4 & 4.4 & & \\
\hline 9.6 & 1.1 & 2.712 & 1982.438 & 9-Jun-82 & 3.5 & 4.4 & & \\
\hline 9.2 & 1.5 & 2.841 & 1982.567 & 26-Jul-82 & 3.2 & 4.3 & & \\
\hline 8.5 & 1.9 & 2.918 & 1982.644 & 23-Aug-82 & 2.9 & 4.1 & & \\
\hline 10.4 & 1.1 & 3.321 & 1983.047 & 17-Jan-83 & 3.1 & 4.0 & & \\
\hline 21.0 & 1.1 & 3.660 & 1983.386 & 21-May-83 & 5.7 & 4.8 & & \\
\hline 19.0 & 1.0 & 3.690 & 1983.416 & 1-Jun-83 & 5.1 & 5.1 & & \\
\hline 20.0 & 0.7 & 3.718 & 1983.444 & 11-Jun-83 & 5.4 & 5.3 & & \\
\hline 19.3 & 1.1 & 3.871 & 1983.597 & 6-Aug-83 & 5.0 & 5.5 & & \\
\hline 20.4 & 1.9 & 4.121 & 1983.847 & 5-Nov-83 & 5.0 & 5.6 & & \\
\hline 22.2 & 1.3 & 4.370 & 1984.096 & 4-Feb-84 & 5.1 & 5.6 & & \\
\hline 23.9 & 0.7 & 4.624 & 1984.350 & 7-May-84 & 5.2 & 5.7 & & \\
\hline 23.5 & 0.3 & 4.714 & 1984.440 & 9-Jun-84 & 5.0 & 5.7 & & \\
\hline 22.9 & 1.3 & 4.867 & 1984.593 & 4-Aug-84 & 4.7 & 5.7 & & \\
\hline 23.3 & 1.0 & 5.039 & 1984.765 & $6-O c t-84$ & 4.6 & 5.7 & & \\
\hline 24.0 & 0.8 & 5.192 & 1984.918 & 1-Dec-84 & 4.6 & 5.6 & & \\
\hline 26.9 & 0.4 & 5.367 & 1985.093 & 3-Feb-85 & 5.0 & 5.6 & & \\
\hline 25.5 & 0.2 & 5.600 & 1985.326 & 29-Apr-85 & 4.6 & 5.6 & & \\
\hline 26.3 & 0.3 & 5.732 & 1985.458 & 16-Jun-85 & 4.6 & 5.5 & & \\
\hline 26.0 & 0.2 & 6.082 & 1985.808 & $22-$-ct-85 & 4.3 & 5.4 & & \\
\hline 29.0 & 0.2 & 6.225 & 1985.951 & 13-Dec-85 & 4.7 & 5.4 & & \\
\hline 37.0 & 1.6 & 6.458 & 1986.184 & 8-Mar-86 & 5.7 & 5.5 & & \\
\hline 39.0 & 0.6 & 6.521 & 1986.247 & 31-Mar-86 & 6.0 & 5.7 & & \\
\hline 37.8 & 0.5 & 6.696 & 1986.422 & 3-Jun-86 & 5.6 & 5.8 & & \\
\hline 38.5 & 0.5 & 6.806 & 1986.532 & 13-Jul-86 & 5.7 & 5.8 & & \\
\hline 34.5 & 1.0 & 6.956 & 1986.682 & 6-Sep-86 & 5.0 & 5.8 & & \\
\hline 34.7 & 1.7 & 7.090 & 1986.816 & $25-$-Oct-86 & 4.9 & 5.8 & & \\
\hline 34.0 & 1.9 & 7.225 & 1986.951 & 13-Dec-86 & 4.7 & 5.7 & & \\
\hline 38.1 & 1.3 & 7.512 & 1987.238 & 28-Mar-87 & 5.1 & 5.7 & & \\
\hline 38.9 & 1.8 & 7.630 & 1987.356 & 10-May-87 & 5.1 & 5.7 & & \\
\hline 40.3 & 1.7 & 7.800 & 1987.526 & 11-Jul-87 & 5.2 & 5.7 & & \\
\hline
\end{tabular}




\begin{tabular}{|c|c|c|c|c|c|c|c|c|}
\hline 35.3 & 1.8 & 8.126 & 1987.852 & 7-Nov-87 & 4.3 & 5.6 & & \\
\hline 39.8 & 0.8 & 8.340 & 1988.066 & 24-Jan-88 & 4.8 & 5.5 & & \\
\hline 40.5 & 0.6 & 8.566 & 1988.292 & 16-Apr-88 & 4.7 & 5.5 & & \\
\hline 48.6 & 1.2 & 8.684 & 1988.410 & 29-Мay-88 & 5.6 & 5.5 & & \\
\hline 47.8 & 1.0 & 8.719 & 1988.445 & 11-Jun-88 & 5.5 & 5.6 & & \\
\hline 47.8 & 2.5 & 8.872 & 1988.598 & 6-Aug-88 & 5.4 & 5.6 & & \\
\hline 45.4 & 2.1 & 9.083 & 1988.809 & $22-0 c t-88$ & 5.0 & 5.6 & & \\
\hline 45.2 & 2.3 & 9.219 & 1988.945 & 11-Dec-88 & 4.9 & 5.6 & & \\
\hline 47.9 & 1.5 & 9.392 & 1989.118 & 12-Feb-89 & 5.1 & 5.5 & & \\
\hline 48.6 & 0.3 & 9.523 & 1989.249 & 1-Apr-89 & 5.1 & 5.5 & & \\
\hline 47.4 & 1.2 & 9.715 & 1989.441 & 10-Jun-89 & 4.9 & 5.5 & & \\
\hline 44.7 & 0.9 & 9.869 & 1989.595 & 5-Aug-89 & 4.5 & 5.5 & & \\
\hline 46.8 & 1.0 & 10.082 & 1989.808 & 22-Oct-89 & 4.6 & & & \\
\hline 47.6 & 0.9 & 10.271 & 1989.997 & 30-Dec-89 & 4.6 & & & \\
\hline 47.8 & 1.2 & 10.386 & 1990.112 & 10-Feb-90 & 4.6 & & & \\
\hline 51.1 & 1.0 & 10.501 & 1990.227 & 24-Mar-90 & 4.9 & & & \\
\hline 53.3 & 1.3 & 10.616 & 1990.342 & 5-May-90 & 5.0 & & & \\
\hline 53.4 & 1.7 & 10.770 & 1990.496 & 30-Jun-90 & 5.0 & & & \\
\hline 51.2 & 1.5 & 10.852 & 1990.578 & 30-Jul-90 & 4.7 & & & \\
\hline 51.8 & 1.5 & 10.975 & 1990.701 & 13-Sep-90 & 4.7 & & & \\
\hline 49.8 & 1.8 & 11.079 & 1990.805 & 21-Oct-90 & 4.5 & & 4.8 & 3.0 \\
\hline 50.7 & 1.5 & 11.323 & 1991.049 & 18-Jan-91 & 4.5 & & 3.4 & 3.1 \\
\hline 50.7 & 1.8 & 11.463 & 1991.189 & 10-Mar-91 & 4.4 & & 2.5 & 2.8 \\
\hline 50.2 & 1.9 & 11.633 & 1991.359 & 11-May-91 & 4.3 & & 1.8 & 2.2 \\
\hline 53.2 & 0.8 & 11.825 & 1991.551 & 20-Jul-91 & 4.5 & & 2.1 & 3.7 \\
\hline 49.9 & 0.6 & 11.942 & 1991.668 & 1-Sep-91 & 4.2 & & 1.5 & 1.7 \\
\hline 53.2 & 0.6 & 12.132 & 1991.858 & 9-Nov-91 & 4.4 & & 1.6 & 3.1 \\
\hline 54.3 & 1.8 & 12.320 & 1992.046 & 17-Jan-92 & 4.4 & & 1.9 & 3.4 \\
\hline 55.9 & 1.4 & 12.514 & 1992.240 & 28-Mar-92 & 4.5 & & 2.2 & 3.7 \\
\hline 55.8 & 1.5 & 12.689 & 1992.415 & 31-May-92 & 4.4 & & 2.4 & 3.5 \\
\hline 55.0 & 2.3 & 12.842 & 1992.568 & 26-Jul-92 & 4.3 & & 2.4 & 3.0 \\
\hline 50.3 & 0.9 & 13.014 & 1992.740 & 27-Sep-92 & 3.9 & & 1.9 & 1.2 \\
\hline 48.4 & 0.9 & 13.165 & 1992.891 & 21-Nov-92 & 3.7 & & 1.3 & 0.5 \\
\hline 52.1 & 1.8 & 13.315 & 1993.041 & 15-Jan-93 & 3.9 & & 1.2 & 1.6 \\
\hline 53.2 & 0.8 & 13.452 & 1993.178 & 6-Mar-93 & 4.0 & & 1.1 & 1.9 \\
\hline 65.1 & 1.1 & 13.625 & 1993.351 & 8-May-93 & 4.8 & & 1.9 & 5.2 \\
\hline 63.8 & 0.9 & 13.836 & 1993.562 & 24-Jul-93 & 4.6 & & 2.4 & 4.5 \\
\hline 59.3 & 0.9 & 14.027 & 1993.753 & 2-Oct-93 & 4.2 & & 2.4 & 3.2 \\
\hline 60.3 & 0.7 & 14.219 & 1993.945 & 11-Dec-93 & 4.2 & & 2.5 & 3.3 \\
\hline 63.8 & 0.8 & 14.395 & 1994.121 & 13-Feb-94 & 4.4 & & 2.8 & 3.9 \\
\hline 63.4 & 0.9 & 14.548 & 1994.274 & 10-Apr-94 & 4.4 & & 2.9 & 3.7 \\
\hline 69.9 & 0.5 & 14.718 & 1994.444 & 11-Jun-94 & 4.7 & & 3.3 & 5.0 \\
\hline 69.8 & 1.3 & 14.866 & 1994.592 & 4-Aug-94 & 4.7 & & 3.5 & 4.8 \\
\hline 68.4 & 0.8 & 15.044 & 1994.770 & 8-Oct-94 & 4.5 & & 3.7 & 4.4 \\
\hline 70.3 & 1.9 & 15.236 & 1994.962 & 17-Dec-94 & 4.6 & & 3.8 & 4.6 \\
\hline 69.6 & 1.0 & 15.427 & 1995.153 & 25-Feb-95 & 4.5 & & 3.9 & 4.3 \\
\hline 73.4 & 1.1 & 15.600 & 1995.326 & 29-Apr-95 & 4.7 & & 4.1 & 4.8 \\
\hline 72.3 & 1.1 & 15.756 & 1995.482 & 25-Jun-95 & 4.6 & & 4.2 & 4.5 \\
\hline 71.9 & 0.8 & 15.921 & 1995.647 & 24-Aug-95 & 4.5 & & 4.2 & 4.3 \\
\hline
\end{tabular}




\begin{tabular}{|c|c|c|c|c|c|c|c|}
\hline 70.5 & 3.4 & 16.082 & 1995.808 & $22-$ Oct-95 & 4.4 & 4.2 & 4.0 \\
\hline 71.4 & 1.2 & 16.233 & 1995.959 & 16-Dec-95 & 4.4 & 4.2 & 4.0 \\
\hline 76.4 & 1.3 & 16.405 & 1996.131 & 17-Feb-96 & 4.7 & 4.3 & 4.7 \\
\hline 80.5 & 3.5 & 16.635 & 1996.361 & 11-May-96 & 4.8 & 4.4 & 5.1 \\
\hline 74.8 & 3.0 & 16.807 & 1996.533 & 13-Jul-96 & 4.5 & 4.4 & 4.2 \\
\hline 72.9 & 0.3 & 16.913 & 1996.639 & 21-Aug-96 & 4.3 & 4.4 & 3.8 \\
\hline 75.2 & 1.1 & 17.075 & 1996.801 & 19-Oct-96 & 4.4 & 4.4 & 4.1 \\
\hline 75.8 & 0.9 & 17.228 & 1996.954 & 14-Dec-96 & 4.4 & 4.4 & 4.1 \\
\hline 79.5 & 0.9 & 17.381 & 1997.107 & 8-Feb-97 & 4.6 & 4.4 & 4.5 \\
\hline 78.8 & 0.8 & 17.592 & 1997.318 & 26-Apr-97 & 4.5 & 4.4 & 4.3 \\
\hline 86.7 & 1.5 & 17.745 & 1997.471 & 21-Jun-97 & 4.9 & 4.5 & 5.2 \\
\hline 84.2 & 0.3 & 17.918 & 1997.644 & 23-Aug-97 & 4.7 & 4.6 & 4.8 \\
\hline 83.3 & 1.6 & 18.071 & 1997.797 & 18-Oct-97 & 4.6 & 4.6 & 4.6 \\
\hline 87.7 & 2.0 & 18.263 & 1997.989 & 27-Dec-97 & 4.8 & 4.7 & 5.0 \\
\hline 90.5 & 0.9 & 18.419 & 1998.145 & 22-Feb-98 & 4.9 & 4.8 & 5.2 \\
\hline 91.1 & 1.7 & 18.589 & 1998.315 & 25-Apr-98 & 4.9 & 4.8 & 5.2 \\
\hline 90.0 & 0.9 & 18.726 & 1998.452 & 14-Jun-98 & 4.8 & 4.9 & 5.0 \\
\hline 91.1 & 0.4 & 18.956 & 1998.682 & 6-Sep-98 & 4.8 & 4.9 & 5.0 \\
\hline 88.7 & 0.5 & 19.164 & 1998.890 & 21-Nov-98 & 4.6 & 4.9 & 4.6 \\
\hline 94.5 & 0.3 & 19.356 & 1999.082 & 30-Jan-99 & 4.9 & 5.0 & 5.1 \\
\hline 96.9 & 1.2 & 19.493 & 1999.219 & 21-Mar-99 & 5.0 & 5.0 & 5.3 \\
\hline 95.9 & 2.2 & 19.663 & 1999.389 & 22-Мay-99 & 4.9 & 5.1 & 5.1 \\
\hline 92.2 & 0.7 & 19.836 & 1999.562 & 24-Jul-99 & 4.6 & 5.1 & 4.7 \\
\hline 91.2 & 1.2 & 19.973 & 1999.699 & 12-Sep-99 & 4.6 & 5.1 & 4.5 \\
\hline 92.4 & 0.8 & 20.162 & 1999.888 & 20-Nov-99 & 4.6 & 5.0 & 4.5 \\
\hline 94.4 & 0.8 & 20.334 & 2000.060 & 22-Jan-00 & 4.6 & 5.0 & 4.6 \\
\hline 107.1 & 0.6 & 20.506 & 2000.232 & 25-Mar-00 & 5.2 & 5.1 & 5.8 \\
\hline 107.2 & 0.6 & 20.662 & 2000.388 & 21-May-00 & 5.2 & 5.2 & 5.7 \\
\hline 108.2 & 1.7 & 20.851 & 2000.577 & 29-Jul-00 & 5.2 & 5.3 & 5.7 \\
\hline 106.2 & 2.4 & 21.080 & 2000.806 & 21-Oct-00 & 5.0 & 5.3 & 5.4 \\
\hline 107.0 & 1.2 & 21.271 & 2000.997 & 30-Dec-00 & 5.0 & 5.4 & 5.4 \\
\hline
\end{tabular}


SF-02 Appian Way in Union City Hayward Fault 55.65 km

\begin{tabular}{|c|c|c|c|c|c|}
\hline $\begin{array}{l}\text { movement } \\
(\mathrm{mm})\end{array}$ & std. dev. & \# of years & year & date & $\begin{array}{c}\mathrm{mm} / \mathrm{yr}-\text { simple } \\
\text { average }\end{array}$ \\
\hline 0.0 & 1.7 & 0.000 & 1979.729 & 23-Sep-79 & \\
\hline-2.0 & 3.0 & 0.131 & 1979.860 & 10-Nov-79 & \\
\hline-4.4 & 1.5 & 0.421 & 1980.150 & 24-Feb-80 & \\
\hline-4.7 & 1.9 & 0.555 & 1980.284 & 13-Apr-80 & \\
\hline 2.2 & 2.5 & 0.807 & 1980.536 & 14-Jul-80 & \\
\hline 2.7 & 2.2 & 0.990 & 1980.719 & 19-Sep-80 & 2.7 \\
\hline 3.7 & 2.5 & 1.203 & 1980.932 & 6-Dec-80 & 3.1 \\
\hline 5.0 & 2.9 & 1.307 & 1981.036 & 13-Jan-81 & 3.8 \\
\hline 2.8 & 0.9 & 1.452 & 1981.181 & 7-Mar-81 & 1.9 \\
\hline 5.2 & 1.5 & 1.709 & 1981.438 & 9-Jun-81 & 3.0 \\
\hline 5.9 & 0.6 & 1.868 & 1981.597 & 6-Aug-81 & 3.2 \\
\hline 9.9 & 1.2 & 2.200 & 1981.929 & 5-Dec-81 & 4.5 \\
\hline 9.9 & 1.4 & 2.353 & 1982.082 & 30-Jan-82 & 4.2 \\
\hline 10.8 & 1.0 & 2.468 & 1982.197 & 13-Mar-82 & 4.4 \\
\hline 10.8 & 0.7 & 2.709 & 1982.438 & 9-Jun-82 & 4.0 \\
\hline 12.3 & 0.7 & 2.838 & 1982.567 & 26-Jul-82 & 4.3 \\
\hline 12.0 & 1.0 & 2.915 & 1982.644 & 23-Aug-82 & 4.1 \\
\hline 16.4 & 1.2 & 3.181 & 1982.910 & 28-Nov-82 & 5.2 \\
\hline 16.6 & 0.9 & 3.318 & 1983.047 & 17-Jan-83 & 5.0 \\
\hline 14.7 & 1.5 & 3.657 & 1983.386 & 21-May-83 & 4.0 \\
\hline 16.1 & 0.7 & 3.715 & 1983.444 & 11-Jun-83 & 4.3 \\
\hline 17.6 & 1.1 & 3.868 & 1983.597 & 6-Aug-83 & 4.5 \\
\hline 19.1 & 1.4 & 4.118 & 1983.847 & 5-Nov-83 & 4.6 \\
\hline 18.5 & 1.4 & 4.369 & 1984.098 & 5-Feb-84 & 4.2 \\
\hline 20.5 & 1.0 & 4.621 & 1984.350 & 7-Мay-84 & 4.4 \\
\hline 22.4 & 0.4 & 4.711 & 1984.440 & 9-Jun-84 & 4.8 \\
\hline 22.7 & 1.8 & 4.864 & 1984.593 & 4-Aug-84 & 4.7 \\
\hline 22.4 & 0.3 & 5.036 & 1984.765 & $6-0 c t-84$ & 4.4 \\
\hline 23.4 & 0.4 & 5.189 & 1984.918 & 1-Dec-84 & 4.5 \\
\hline 22.7 & 0.8 & 5.364 & 1985.093 & 3-Feb-85 & 4.2 \\
\hline 25.3 & 0.6 & 5.597 & 1985.326 & 29-Apr-85 & 4.5 \\
\hline 26.7 & 0.8 & 5.770 & 1985.499 & 1-Jul-85 & 4.6 \\
\hline 28.7 & 0.1 & 5.994 & 1985.723 & 21-Sep-85 & 4.8 \\
\hline 28.3 & 0.8 & 6.222 & 1985.951 & 13-Dec-85 & 4.5 \\
\hline 30.3 & 0.3 & 6.457 & 1986.186 & 9-Mar-86 & 4.7 \\
\hline 30.1 & 0.5 & 6.518 & 1986.247 & 31-Mar-86 & 4.6 \\
\hline 29.4 & 0.8 & 6.592 & 1986.321 & $27-A p r-86$ & 4.5 \\
\hline 32.8 & 1.6 & 6.693 & 1986.422 & 3-Jun-86 & 4.9 \\
\hline 31.0 & 1.0 & 6.803 & 1986.532 & 13-Jul-86 & 4.6 \\
\hline 28.4 & 1.1 & 6.953 & 1986.682 & 6-Sep-86 & 4.1 \\
\hline 30.4 & 1.7 & 7.087 & 1986.816 & $25-$ Oct- 86 & 4.3 \\
\hline 29.9 & 1.1 & 7.222 & 1986.951 & 13-Dec-86 & 4.1 \\
\hline 34.2 & 1.9 & 7.509 & 1987.238 & 28-Mar-87 & 4.6 \\
\hline 36.0 & 2.3 & 7.627 & 1987.356 & 10-May-87 & 4.7 \\
\hline 32.2 & 1.0 & 7.797 & 1987.526 & 11-Jul-87 & 4.1 \\
\hline
\end{tabular}




\begin{tabular}{|c|c|c|c|c|c|}
\hline 33.2 & 1.5 & 8.123 & 1987.852 & 7-Nov-87 & 4.1 \\
\hline 35.9 & 1.1 & 8.337 & 1988.066 & 24-Jan-88 & 4.3 \\
\hline 36.2 & 1.0 & 8.563 & 1988.292 & 16-Apr-88 & 4.2 \\
\hline 37.4 & 1.0 & 8.681 & 1988.410 & 29-May-88 & 4.3 \\
\hline 36.0 & 1.2 & 8.716 & 1988.445 & 11-Jun-88 & 4.1 \\
\hline 37.9 & 1.4 & 8.869 & 1988.598 & 6-Aug-88 & 4.3 \\
\hline 37.0 & 0.7 & 9.080 & 1988.809 & $22-0 c t-88$ & 4.1 \\
\hline 36.6 & 1.0 & 9.216 & 1988.945 & 11-Dec-88 & 4.0 \\
\hline 42.8 & 1.0 & 9.389 & 1989.118 & 12-Feb-89 & 4.6 \\
\hline 45.3 & 1.6 & 9.520 & 1989.249 & 1-Apr-89 & 4.8 \\
\hline 46.6 & 0.6 & 9.712 & 1989.441 & 10-Jun-89 & 4.8 \\
\hline 45.3 & 1.2 & 9.866 & 1989.595 & 5-Aug-89 & 4.6 \\
\hline 44.7 & 0.6 & 10.079 & 1989.808 & 22-Oct-89 & 4.4 \\
\hline 45.9 & 1.3 & 10.268 & 1989.997 & 30-Dec-89 & 4.5 \\
\hline 46.3 & 0.9 & 10.383 & 1990.112 & 10-Feb-90 & 4.5 \\
\hline 47.0 & 0.4 & 10.498 & 1990.227 & 24-Mar-90 & 4.5 \\
\hline 51.9 & 1.2 & 10.613 & 1990.342 & 5-May-90 & 4.9 \\
\hline 50.1 & 1.0 & 10.767 & 1990.496 & 30-Jun-90 & 4.7 \\
\hline 49.4 & 1.2 & 10.846 & 1990.575 & 29-Jul-90 & 4.6 \\
\hline 47.9 & 1.2 & 10.972 & 1990.701 & 13-Sep-90 & 4.4 \\
\hline 50.0 & 1.4 & 11.076 & 1990.805 & 21-Oct-90 & 4.5 \\
\hline 45.9 & 1.6 & 11.320 & 1991.049 & 18-Jan-91 & 4.1 \\
\hline 48.8 & 0.4 & 11.512 & 1991.241 & 29-Mar-91 & 4.2 \\
\hline 51.9 & 1.0 & 11.630 & 1991.359 & 11-May-91 & 4.5 \\
\hline 51.3 & 1.1 & 11.939 & 1991.668 & 1-Sep-91 & 4.3 \\
\hline 53.5 & 1.4 & 12.129 & 1991.858 & 9-Nov-91 & 4.4 \\
\hline 52.8 & 1.0 & 12.317 & 1992.046 & 17-Jan-92 & 4.3 \\
\hline 59.1 & 1.2 & 12.511 & 1992.240 & 28-Mar-92 & 4.7 \\
\hline 59.6 & 0.6 & 12.686 & 1992.415 & 31-May-92 & 4.7 \\
\hline 60.4 & 1.2 & 12.839 & 1992.568 & 26-Jul-92 & 4.7 \\
\hline 58.2 & 1.6 & 13.011 & 1992.740 & 27-Sep-92 & 4.5 \\
\hline 61.0 & 1.4 & 13.162 & 1992.891 & 21-Nov-92 & 4.6 \\
\hline 61.6 & 1.6 & 13.312 & 1993.041 & 15-Jan-93 & 4.6 \\
\hline 61.7 & 1.2 & 13.449 & 1993.178 & 6-Mar-93 & 4.6 \\
\hline 60.7 & 1.9 & 13.622 & 1993.351 & 8-May-93 & 4.5 \\
\hline 62.5 & 1.5 & 13.709 & 1993.438 & 9-Jun-93 & 4.6 \\
\hline 64.1 & 0.9 & 13.909 & 1993.638 & 21-Aug-93 & 4.6 \\
\hline 65.6 & 1.3 & 14.044 & 1993.773 & 9-Oct-93 & 4.7 \\
\hline 66.9 & 4.7 & 14.233 & 1993.962 & 17-Dec-93 & 4.7 \\
\hline 71.5 & 1.4 & 14.430 & 1994.159 & 27-Feb-94 & 5.0 \\
\hline 71.3 & 1.3 & 14.583 & 1994.312 & 24-Apr-94 & 4.9 \\
\hline 74.1 & 3.8 & 14.813 & 1994.542 & 17-Jul-94 & 5.0 \\
\hline 73.2 & 2.9 & 15.079 & 1994.808 & 22-Oct-94 & 4.9 \\
\hline 73.2 & 3.6 & 15.348 & 1995.007 & 28-Jan-95 & 4.8 \\
\hline 76.2 & 1.6 & 15.539 & 1995.268 & 8-Apr-95 & 4.9 \\
\hline 77.4 & 1.4 & 15.731 & 1995.460 & 17-Jun-95 & 4.9 \\
\hline 77.7 & 4.6 & 15.918 & 1995.647 & 24-Aug-95 & 4.9 \\
\hline 79.1 & 1.6 & 16.096 & 1995.825 & 28-Oct-95 & 4.9 \\
\hline 82.1 & 1.6 & 16.249 & 1995.978 & 23-Dec-95 & 5.1 \\
\hline
\end{tabular}




$\begin{array}{cccccc}79.8 & 1.7 & 16.386 & 1996.115 & \text { 11-Feb-96 } & 4.9 \\ 81.1 & 1.0 & 16.424 & 1996.153 & \text { 25-Feb-96 } & 4.9 \\ 80.0 & 0.9 & 16.536 & 1996.265 & \text { 6-Apr-96 } & 4.8 \\ 84.9 & 3.5 & 16.766 & 1996.495 & \text { 29-Jun-96 } & 5.1 \\ 83.3 & 0.5 & 16.910 & 1996.639 & \text { 21-Aug-96 } & 4.9 \\ 85.7 & 1.4 & 17.091 & 1996.820 & \text { 22-Oct-96 } & 5.0 \\ 88.0 & 1.4 & 17.244 & 1996.973 & 21 \text {-Dec-96 } & 5.1 \\ 86.0 & 1.4 & 17.400 & 1997.129 & 16 \text {-Feb-97 } & 4.9 \\ 87.6 & 0.8 & 17.589 & 1997.318 & \text { 26-Apr-97 } & 5.0 \\ 90.1 & 0.4 & 17.742 & 1997.471 & \text { 21-Jun-97 } & 5.1 \\ 91.4 & 0.7 & 17.915 & 1997.644 & \text { 23-Aug-97 } & 5.1 \\ 91.3 & 0.7 & 18.126 & 1997.855 & \text { 8-Nov-97 } & 5.0 \\ 95.6 & 1.1 & 18.298 & 1998.027 & \text { 10-Jan-98 } & 5.2 \\ 89.7 & 2.3 & 18.471 & 1998.200 & \text { 14-Mar-98 } & 4.9 \\ 93.7 & 1.4 & 18.759 & 1998.488 & \text { 27-Jun-98 } & 5.0 \\ 96.1 & 4.1 & 18.953 & 1998.682 & \text { 6-Sep-98 } & 5.1 \\ 99.6 & 0.9 & 19.126 & 1998.855 & \text { 8-Nov-98 } & 5.2 \\ 99.5 & 0.9 & 19.413 & 1999.142 & \text { 21-Feb-99 } & 5.1 \\ 101.5 & 0.5 & 19.586 & 1999.315 & \text { 25-Apr-99 } & 5.2 \\ 100.1 & 1.1 & 19.718 & 1999.447 & \text { 12-Jun-99 } & 5.1 \\ 102.9 & 0.6 & 19.929 & 1999.658 & \text { 28-Aug-99 } & 5.2 \\ 98.8 & 1.4 & 20.101 & 1999.830 & \text { 30-Oct-99 } & 4.9 \\ 105.7 & 0.8 & 20.312 & 2000.041 & \text { 15-Jan-00 } & 5.2 \\ 105.8 & 1.5 & 20.484 & 2000.213 & \text { 18-Mar-00 } & 5.2 \\ 107.4 & 1.3 & 20.675 & 2000.404 & \text { 27-May-00 } & 5.2 \\ 107.5 & 0.6 & 20.850 & 2000.579 & \text { 30-Jul-00 } & 5.2 \\ 112.4 & 1.6 & 21.099 & 2000.828 & \text { 29-Oct-00 } & 5.3 \\ 115.5 & 0.9 & 21.268 & 2000.997 & \text { 30-Dec-00 } & 5.4\end{array}$




\section{SF-03 Ashbury Drive in Concord Concord Fault}

\begin{tabular}{|c|c|c|c|c|c|}
\hline $\begin{array}{l}\text { movement } \\
(\mathrm{mm})\end{array}$ & std. dev. & \# of years & year & date & $\begin{array}{c}\mathrm{mm} / \mathrm{yr} \text {-simple } \\
\text { average }\end{array}$ \\
\hline 0.0 & 1.7 & 0.000 & 1979.742 & 28-Sep-79 & \\
\hline 3.3 & 1.2 & 0.042 & 1979.784 & 13-Oct-79 & \\
\hline 9.8 & 0.8 & 0.173 & 1979.915 & $30-N o v-79$ & \\
\hline 11.0 & 1.2 & 0.283 & 1980.025 & 9-Jan-80 & \\
\hline 10.1 & 1.5 & 0.332 & 1980.074 & 27-Jan-80 & \\
\hline 10.5 & 1.6 & 0.351 & 1980.093 & 3-Feb-80 & \\
\hline 9.4 & 1.4 & 0.774 & 1980.516 & 7-Jul-80 & \\
\hline 10.7 & 1.2 & 0.963 & 1980.705 & 14-Sep-80 & 11.1 \\
\hline 11.7 & 1.4 & 1.116 & 1980.858 & 9-Nov-80 & 10.5 \\
\hline 13.4 & 2.3 & 1.250 & 1980.992 & 28-Dec-80 & 10.7 \\
\hline 12.7 & 0.6 & 1.365 & 1981.107 & 8-Feb-81 & 9.3 \\
\hline 10.6 & 0.7 & 1.631 & 1981.373 & 16-May-81 & 6.5 \\
\hline 10.7 & 0.5 & 1.710 & 1981.452 & 14-Jun-81 & 6.3 \\
\hline 14.6 & 1.2 & 1.863 & 1981.605 & 9-Aug-81 & 7.8 \\
\hline 12.7 & 1.2 & 2.305 & 1982.047 & 17-Jan-82 & 5.5 \\
\hline 13.7 & 1.9 & 2.496 & 1982.238 & 28-Mar-82 & 5.5 \\
\hline 13.1 & 0.5 & 2.707 & 1982.449 & 13-Jun-82 & 4.8 \\
\hline 14.6 & 1.0 & 2.822 & 1982.564 & 25-Jul-82 & 5.2 \\
\hline 10.3 & 1.3 & 2.976 & 1982.718 & 19-Sep-82 & 3.5 \\
\hline 10.5 & 0.9 & 3.053 & 1982.795 & $17-$ Oct-82 & 3.4 \\
\hline 14.6 & 2.1 & 3.206 & 1982.948 & $12-\mathrm{Dec}-82$ & 4.6 \\
\hline 14.4 & 1.2 & 3.417 & 1983.159 & 27-Feb-83 & 4.2 \\
\hline 14.2 & 1.3 & 3.647 & 1983.389 & 22-Мay-83 & 3.9 \\
\hline 13.9 & 1.4 & 3.858 & 1983.600 & 7-Aug-83 & 3.6 \\
\hline 14.5 & 1.1 & 4.028 & 1983.770 & 8-Oct-83 & 3.6 \\
\hline 15.5 & 1.3 & 4.184 & 1983.926 & 4-Dec-83 & 3.7 \\
\hline 15.1 & 1.3 & 4.318 & 1984.060 & 22-Jan-84 & 3.5 \\
\hline 14.1 & 2.3 & 4.490 & 1984.232 & 25-Mar-84 & 3.1 \\
\hline 17.0 & 0.7 & 4.720 & 1984.462 & 17-Jun-84 & 3.6 \\
\hline 21.6 & 2.1 & 4.911 & 1984.653 & 26-Aug-84 & 4.4 \\
\hline 21.1 & 1.9 & 4.949 & 1984.691 & 9-Sep-84 & 4.3 \\
\hline 23.5 & 0.7 & 5.045 & 1984.787 & 14-Oct-84 & 4.7 \\
\hline 24.2 & 0.6 & 5.141 & 1984.883 & 18-Nov-84 & 4.7 \\
\hline 23.3 & 0.4 & 5.318 & 1985.060 & 22-Jan-85 & 4.4 \\
\hline 20.4 & 0.7 & 5.466 & 1985.208 & 17-Mar-85 & 3.7 \\
\hline 22.4 & 0.1 & 5.677 & 1985.419 & 2-Jun-85 & 3.9 \\
\hline 23.0 & 0.8 & 5.792 & 1985.534 & 14-Jul-85 & 4.0 \\
\hline 25.7 & 0.3 & 6.022 & 1985.764 & $6-0 c t-85$ & 4.3 \\
\hline 25.7 & 0.8 & 6.483 & 1986.225 & 23-Mar-86 & 4.0 \\
\hline 25.4 & 1.4 & 6.713 & 1986.455 & 15-Jun-86 & 3.8 \\
\hline 27.9 & 0.7 & 6.847 & 1986.589 & 3-Aug-86 & 4.1 \\
\hline 23.7 & 1.5 & 7.077 & 1986.819 & $26-0 c t-86$ & 3.3 \\
\hline 27.1 & 0.8 & 7.346 & 1987.088 & 1-Feb-87 & 3.7 \\
\hline 27.1 & 0.5 & 7.710 & 1987.452 & 14-Jun-87 & 3.5 \\
\hline 26.7 & 0.9 & 7.902 & 1987.644 & 23-Aug-87 & 3.4 \\
\hline 27.3 & 0.4 & 8.170 & 1987.912 & 29-Nov-87 & 3.3 \\
\hline
\end{tabular}




\begin{tabular}{|c|c|c|c|c|c|}
\hline 35.2 & 1.2 & 8.400 & 1988.142 & 21-Feb-88 & 4.2 \\
\hline 35.3 & 0.9 & 8.477 & 1988.219 & 20-Mar-88 & 4.2 \\
\hline 36.1 & 1.4 & 8.572 & 1988.314 & 24-Apr-88 & 4.2 \\
\hline 37.0 & 0.9 & 8.783 & 1988.525 & 10-Jul-88 & 4.2 \\
\hline 37.0 & 0.6 & 8.955 & 1988.697 & 11-Sep-88 & 4.1 \\
\hline 39.3 & 0.5 & 9.184 & 1988.926 & 4-Dec-88 & 4.3 \\
\hline 40.2 & 0.7 & 9.337 & 1989.079 & 29-Jan-89 & 4.3 \\
\hline 42.0 & 1.4 & 9.529 & 1989.271 & 9-Apr-89 & 4.4 \\
\hline 42.0 & 1.6 & 9.776 & 1989.518 & 8-Jul-89 & 4.3 \\
\hline 37.8 & 1.0 & 9.894 & 1989.636 & 20-Aug-89 & 3.8 \\
\hline 42.8 & 1.4 & 10.162 & 1989.904 & 26-Nov-89 & 4.2 \\
\hline 44.2 & 0.6 & 10.354 & 1990.096 & 4-Feb-90 & 4.3 \\
\hline 44.4 & 1.1 & 10.450 & 1990.192 & 11-Mar-90 & 4.2 \\
\hline 45.5 & 0.6 & 10.524 & 1990.266 & 7-Apr-90 & 4.3 \\
\hline 44.5 & 0.6 & 10.603 & 1990.345 & 6-May-90 & 4.2 \\
\hline 45.3 & 1.0 & 10.737 & 1990.479 & 24-Jun-90 & 4.2 \\
\hline 42.7 & 1.2 & 10.891 & 1990.633 & 19-Aug-90 & 3.9 \\
\hline 41.3 & 0.4 & 11.025 & 1990.767 & 7-Oct-90 & 3.7 \\
\hline 41.9 & 0.4 & 11.198 & 1990.940 & 9-Dec-90 & 3.7 \\
\hline 44.3 & 0.6 & 11.370 & 1991.112 & 10-Feb-91 & 3.9 \\
\hline 44.8 & 0.9 & 11.524 & 1991.266 & 7-Apr-91 & 3.9 \\
\hline 43.7 & 0.9 & 11.735 & 1991.477 & 23-Jun-91 & 3.7 \\
\hline 43.1 & 1.1 & 11.869 & 1991.611 & 11-Aug-91 & 3.6 \\
\hline 44.0 & 0.6 & 12.003 & 1991.745 & 29-Sep-91 & 3.7 \\
\hline 46.4 & 1.0 & 12.233 & 1991.975 & 22-Dec-91 & 3.8 \\
\hline 48.0 & 0.6 & 12.444 & 1992.186 & 8-Mar-92 & 3.9 \\
\hline 49.0 & 1.7 & 12.597 & 1992.339 & 3-May-92 & 3.9 \\
\hline 48.3 & 0.7 & 12.788 & 1992.530 & 12-Jul-92 & 3.8 \\
\hline 43.6 & 2.0 & 13.037 & 1992.779 & 11-Oct-92 & 3.3 \\
\hline 47.6 & 2.1 & 13.285 & 1993.027 & 10-Jan-93 & 3.6 \\
\hline 53.8 & 2.6 & 13.439 & 1993.181 & 7-Mar-93 & 4.0 \\
\hline 55.6 & 1.1 & 13.631 & 1993.373 & 16-May-93 & 4.1 \\
\hline 53.1 & 2.4 & 13.880 & 1993.622 & 15-Aug-93 & 3.8 \\
\hline 50.8 & 0.6 & 14.033 & 1993.775 & 10-Oct-93 & 3.6 \\
\hline 56.9 & 1.2 & 14.222 & 1993.964 & 18-Dec-93 & 4.0 \\
\hline 56.4 & 1.9 & 14.455 & 1994.197 & 13-Mar-94 & 3.9 \\
\hline 59.4 & 0.8 & 14.628 & 1994.370 & 15-May-94 & 4.1 \\
\hline 56.9 & 1.4 & 14.820 & 1994.562 & 24-Jul-94 & 3.8 \\
\hline 53.0 & 0.6 & 15.009 & 1994.751 & 1-Oct-94 & 3.5 \\
\hline 59.0 & 0.5 & 15.200 & 1994.942 & 10-Dec-94 & 3.9 \\
\hline 61.9 & 0.9 & 15.357 & 1995.099 & 5-Feb-95 & 4.0 \\
\hline 61.5 & 2.0 & 15.433 & 1995.175 & 5-Mar-95 & 4.0 \\
\hline 60.9 & 1.7 & 15.721 & 1995.463 & 18-Jun-95 & 3.9 \\
\hline 60.4 & 1.3 & 15.951 & 1995.693 & 10-Sep-95 & 3.8 \\
\hline 62.7 & 1.2 & 16.294 & 1996.036 & 13-Jan-96 & 3.8 \\
\hline 63.9 & 1.7 & 16.507 & 1996.249 & 31-Mar-96 & 3.9 \\
\hline 63.5 & 2.0 & 16.736 & 1996.478 & 23-Jun-96 & 3.8 \\
\hline 61.8 & 0.2 & 16.908 & 1996.650 & 25-Aug-96 & 3.7 \\
\hline 61.9 & 1.0 & 17.080 & 1996.822 & $27-0 c t-96$ & 3.6 \\
\hline
\end{tabular}




\begin{tabular}{|c|c|c|c|c|c|}
\hline 71.6 & 0.9 & 17.214 & 1996.956 & 15-Dec-96 & 4.2 \\
\hline 72.7 & 1.4 & 17.288 & 1997.030 & 11-Jan-97 & 4.2 \\
\hline 74.2 & 1.4 & 17.463 & 1997.205 & 16-Mar-97 & 4.2 \\
\hline 72.9 & 0.5 & 17.636 & 1997.378 & 18-May-97 & 4.1 \\
\hline 70.4 & 0.7 & 17.770 & 1997.512 & 6-Jul-97 & 4.0 \\
\hline 69.6 & 0.7 & 18.000 & 1997.742 & 28-Sep-97 & 3.9 \\
\hline 72.2 & 0.7 & 18.173 & 1997.915 & 30-Nov-97 & 4.0 \\
\hline 72.5 & 0.7 & 18.307 & 1998.049 & 18-Jan-98 & 4. \\
\hline 73.3 & 0.2 & 18.461 & 1998.203 & 15-Mar-98 & 4.0 \\
\hline 76.1 & 1.4 & 18.691 & 1998.433 & 7-Jun-98 & 4.1 \\
\hline 77.2 & 0.5 & 18.844 & 1998.586 & 2-Aug-98 & 4.1 \\
\hline 72.7 & 0.5 & 19.074 & 1998.816 & 25-Oct-98 & 3.8 \\
\hline 74.5 & 0.7 & 19.228 & 1998.970 & 20-Dec-98 & 3. \\
\hline 76.6 & 1.6 & 19.362 & 1999.104 & 7-Feb-99 & 4. \\
\hline 77.6 & 1.7 & 19.516 & 1999.258 & 4-Apr-99 & 4.0 \\
\hline 76.0 & 0.8 & 19.688 & 1999.430 & 6-Jun-99 & 3.9 \\
\hline 73.9 & 1.8 & 19.861 & 1999.603 & 8-Aug-99 & 3. \\
\hline 71.6 & 1.8 & 20.072 & 1999.814 & 24-Oct-99 & 3. \\
\hline 74.1 & 0.9 & 20.225 & 1999.967 & 19-Dec-99 & 3.7 \\
\hline 77.1 & 1.7 & 20.416 & 2000.158 & 27-Feb-00 & $3 . \varepsilon$ \\
\hline 79.6 & 1.2 & 20.569 & 2000.311 & 23-Apr-00 & 3. \\
\hline 80.0 & 1.1 & 20.742 & 2000.484 & 25-Jun-00 & 3.9 \\
\hline 77.2 & 0.9 & 20.914 & 2000.656 & 27-Aug-00 & 3.7 \\
\hline 76.6 & 1.5 & 21.048 & 2000.790 & $15-0 c t-00$ & 3. \\
\hline 78.9 & 1.3 & 21.201 & 2000.943 & 10-Dec-00 & 3.7 \\
\hline 81.9 & 1.3 & 21.373 & 2001.115 & $11-$ Feb-01 & 3.8 \\
\hline
\end{tabular}


SF-04 Seventh Street in Hollister Calaveras Fault

movement

$(\mathrm{mm})$

0.0

$-0.8$

$-2.2$

4.2

3.3

2.9

3.7

5.2

14.7

23.0

21.7

20.1

18.0

28.9

27.6

29.3

34.3

33.6

32.7

32.4

30.0

30.0

37.4

41.4

40.6

39.7

39.6

38.3

43.9

44.7

44.8

43.3

47.8

47.9

48.3

47.4

49.3

49.2

49.1

47.7

47.1

48.4

49.4

49.6

55.5

56.8 std. dev.

2.1

1.0

0.8

1.0

0.6

0.6

0.9

1.2

0.7

0.8

0.6

0.5

0.3

1.0

0.5

0.3

0.5

0.5

0.5

0.2

0.6

0.6

0.7

0.4

0.3

0.4

0.5

0.8

0.3

0.9

0.5

0.3

0.5

0.8

0.6

0.4

0.7

0.6

1.2

0.8

1.0

0.6

0.4

0.4

0.4

0.4
\# of years

0.000

0.077

0.296

0.422

0.441

0.594

0.728

1.302

1.723

1.896

2.203

2.567

2.836

2.970

3.162

3.411

3.608

3.702

3.841

4.063

4.332

4.572

4.676

4.774

4.864

5.023

5.192

5.326

5.518

5.748

6.000

6.189

6.334

6.381

6.592

6.682

6.860

7.033

7.187

7.417

7.669

7.877

8.145

8.337

8.416

8.586 year date $\begin{gathered}\mathrm{mm} / \mathrm{yr}-\mathrm{simple} \\ \text { average }\end{gathered}$

$1979.745 \quad 29-$ Sep-79

$1979.822 \quad 27-$ Oct-79

1980.041 15-Jan-80

1980.167 1-Mar-80

1980.186 8-Mar-80

1980.339 3-May-80

1980.473 21-Jun-80

1981.047 17-Jan-81

5.1

4.0

8.5

12.1

1981.641

1981.948

1982.312

1982.581

1982.715

1982.907

1983.156

1983.353

1983.447

1983.586

1983.808

1984.077

1984.317

1984.421

1984.519

1984.609

1984.768

1984.937

1985.071

1985.263

1985.493

1985.745

1985.934

1986.079

1986.126

1986.337

1986.427

1986.605

1986.778

1986.932

1987.162

1987.414

1987.622

1987.890

1988.082

1988.161

1988.331
20-Jun-81

22-Aug-81

12-Dec-81

24-Apr-82

31-Jul-82

18-Sep-82

27-Nov-82

26-Feb-83

9-May-83

12-Jun-83

2-Aug-83

22-0ct-83

28-Jan-84

25-Apr-84

2-Jun-84

8-Jul-84

10-Aug-84

7-Oct-84

8-Dec-84

26-Jan-85

6-Apr-85

29-Jun-85

29-Sep-85

7-Dec-85

29-Jan-86

15-Feb-86

3-May-86

5-Jun-86

11-Oct-86

6-Dec-86

28-Feb-87

31-May-87

15-Aug-87

21-Nov-87

30-Jan-88

28-Feb-88

30-Apr-88
9-Aug-86
9.9

7.8

6.3

9.7

8.7

8.6

9.5

9.1

8.5

8.0

6.9

6.6

8.0

8.7

8.3

7.9

7.6

7.2

8.0

7.8

7.5

7.0

7.5

7.5

7.3

7.1

7.2

7.0

6.8

6.4

6.1

6.1

6.1

5.9

6.6

6.6 


\begin{tabular}{|c|c|c|c|c|c|}
\hline 57.5 & 0.7 & 8.709 & 1988.454 & 14-Jun-88 & 6.6 \\
\hline 57.0 & 0.6 & 8.777 & 1988.522 & 9-Jul-88 & 6.5 \\
\hline 63.6 & 1.1 & 9.009 & 1988.754 & $2-0 c t-88$ & 7.1 \\
\hline 64.9 & 1.0 & 9.178 & 1988.923 & 3-Dec-88 & 7.1 \\
\hline 63.4 & 0.7 & 9.370 & 1989.115 & 11-Feb-89 & 6.8 \\
\hline 64.4 & 1.1 & 9.523 & 1989.268 & 8-Apr-89 & 6.8 \\
\hline 66.0 & 0.8 & 9.715 & 1989.460 & 17-Jun-89 & 6.8 \\
\hline 67.5 & 1.1 & 9.888 & 1989.633 & 19-Aug-89 & 6.8 \\
\hline 81.1 & 0.8 & 10.060 & 1989.805 & 21-Oct-89 & 8.1 \\
\hline 78.6 & 0.3 & 10.118 & 1989.863 & 11-Nov-89 & 7.8 \\
\hline 80.8 & 0.6 & 10.195 & 1989.940 & 9-Dec-89 & 7.9 \\
\hline 81.5 & 0.6 & 10.291 & 1990.036 & 13-Jan-90 & 7.9 \\
\hline 79.9 & 0.2 & 10.406 & 1990.151 & 24-Feb-90 & 7.7 \\
\hline 80.6 & 0.4 & 10.540 & 1990.285 & 14-Apr-90 & 7.6 \\
\hline 81.4 & 0.8 & 10.636 & 1990.381 & 19-May-90 & 7.7 \\
\hline 81.7 & 0.6 & 10.773 & 1990.518 & 8-Jul-90 & 7.6 \\
\hline 82.2 & 0.7 & 10.869 & 1990.614 & 12-Aug-90 & 7.6 \\
\hline 83.7 & 0.5 & 10.984 & 1990.729 & 23-Sep-90 & 7.6 \\
\hline 84.4 & 0.2 & 11.096 & 1990.841 & 3-Nov-90 & 7.6 \\
\hline 84.4 & 0.9 & 11.326 & 1991.071 & 26-Jan-91 & 7.5 \\
\hline 84.8 & 0.7 & 11.518 & 1991.263 & 6-Apr-91 & 7.4 \\
\hline 85.9 & 0.7 & 11.748 & 1991.493 & 29-Jun-91 & 7.3 \\
\hline 86.8 & 0.4 & 11.940 & 1991.685 & 7-Sep-91 & 7.3 \\
\hline 89.4 & 1.0 & 12.132 & 1991.877 & 16-Nov-91 & 7.4 \\
\hline 89.1 & 1.3 & 12.310 & 1992.055 & 20-Jan-92 & 7.2 \\
\hline 88.7 & 0.9 & 12.534 & 1992.279 & 11-Apr-92 & 7.1 \\
\hline 91.7 & 0.5 & 12.725 & 1992.470 & 20-Jun-92 & 7.2 \\
\hline 89.9 & 1.9 & 12.881 & 1992.626 & 16-Aug-92 & 7.0 \\
\hline 92.9 & 1.0 & 13.053 & 1992.798 & 18-Oct-92 & 7.1 \\
\hline 92.8 & 1.3 & 13.225 & 1992.970 & 20-Dec-92 & 7.0 \\
\hline 92.9 & 0.8 & 13.299 & 1993.044 & 16-Jan-93 & 7.0 \\
\hline 93.0 & 1.9 & 13.452 & 1993.197 & 13-Mar-93 & 6.9 \\
\hline 93.6 & 1.5 & 13.608 & 1993.353 & 9-May-93 & 6.9 \\
\hline 94.0 & 0.7 & 13.817 & 1993.562 & 24-Jul-93 & 6.8 \\
\hline 95.6 & 1.7 & 13.913 & 1993.658 & 28-Aug-93 & 6.9 \\
\hline 96.8 & 1.4 & 14.085 & 1993.830 & 30-Oct-93 & 6.9 \\
\hline 95.9 & 0.4 & 14.296 & 1994.041 & 15-Jan-94 & 6.7 \\
\hline 94.8 & 1.6 & 14.488 & 1994.233 & 26-Mar-94 & 6.5 \\
\hline 95.7 & 0.4 & 14.680 & 1994.425 & 4-Jun-94 & 6.5 \\
\hline 96.1 & 0.2 & 14.858 & 1994.603 & 8-Aug-94 & 6.5 \\
\hline 101.3 & 0.9 & 15.121 & 1994.866 & 12-Nov-94 & 6.7 \\
\hline 99.2 & 0.3 & 15.315 & 1995.060 & 22-Jan-95 & 6.5 \\
\hline 98.4 & 1.4 & 15.562 & 1995.307 & 22-Apr-95 & 6.3 \\
\hline 98.6 & 0.9 & 15.584 & 1995.329 & 30-Apr-95 & 6.3 \\
\hline 104.8 & 0.4 & 15.792 & 1995.537 & 15-Jul-95 & 6.6 \\
\hline 106.9 & 0.7 & 16.025 & 1995.770 & 8-Oct-95 & 6.7 \\
\hline 115.1 & 0.4 & 16.252 & 1995.997 & 30-Dec-95 & 7.1 \\
\hline 113.3 & 1.4 & 16.444 & 1996.189 & 9-Mar-96 & 6.9 \\
\hline 113.8 & 2.7 & 16.580 & 1996.325 & 28-Apr-96 & 6.9 \\
\hline
\end{tabular}




\begin{tabular}{|c|c|c|c|c|c|}
\hline 112.8 & 1.0 & 16.654 & 1996.399 & 25-May-96 & 6.8 \\
\hline 113.4 & 0.9 & 16.826 & 1996.571 & 27-Jul-96 & 6.7 \\
\hline 111.7 & 0.7 & 16.998 & 1996.743 & 28-Sep-96 & 6.6 \\
\hline 111.6 & 0.6 & 17.132 & 1996.877 & 16-Nov-96 & 6.5 \\
\hline 113.9 & 0.3 & 17.304 & 1997.049 & 18-Jan-97 & 6.6 \\
\hline 113.2 & 0.7 & 17.477 & 1997.222 & 22-Mar-97 & 6.5 \\
\hline 112.3 & 1.1 & 17.611 & 1997.356 & 10-May-97 & 6.4 \\
\hline 112.8 & 0.6 & 17.784 & 1997.529 & 12-Jul-97 & 6.3 \\
\hline 124.1 & 0.9 & 17.978 & 1997.723 & 21-Sep-97 & 6.9 \\
\hline 124.6 & 0.7 & 18.167 & 1997.912 & 29-Nov-97 & 6.9 \\
\hline 124.7 & 1.1 & 18.340 & 1998.085 & 31-Jan-98 & 6.8 \\
\hline 124.5 & 0.9 & 18.496 & 1998.241 & 29-Mar-98 & 6.7 \\
\hline 123.0 & 1.1 & 18.666 & 1998.411 & 30-May-98 & 6.6 \\
\hline 122.4 & 1.4 & 18.819 & 1998.564 & 25-Jul-98 & 6.5 \\
\hline 124.6 & 0.4 & 18.877 & 1998.622 & 15-Aug-98 & 6.6 \\
\hline 127.2 & 0.9 & 19.088 & 1998.833 & 31-Oct-98 & 6.7 \\
\hline 128.0 & 0.5 & 19.280 & 1999.025 & 9-Jan-99 & 6.6 \\
\hline 127.1 & 0.8 & 19.436 & 1999.181 & 7-Mar-99 & 6.5 \\
\hline 126.7 & 1.1 & 19.606 & 1999.351 & 8-Мay-99 & 6.5 \\
\hline 127.3 & 0.2 & 19.778 & 1999.523 & 10-Jul-99 & 6.4 \\
\hline 127.2 & 0.6 & 19.989 & 1999.734 & 25-Sep-99 & 6.4 \\
\hline 127.3 & 0.3 & 20.162 & 1999.907 & 27-Nov-99 & 6.3 \\
\hline 139.1 & 0.3 & 20.353 & 2000.098 & 5-Feb-00 & 6.8 \\
\hline 140.1 & 0.6 & 20.525 & 2000.270 & 8-Apr-00 & 6.8 \\
\hline 138.9 & 0.1 & 20.698 & 2000.443 & 10-Jun-00 & 6.7 \\
\hline 138.8 & 0.2 & 20.889 & 2000.634 & 19-Aug-00 & 6.6 \\
\hline 141.1 & 0.2 & 21.042 & 2000.787 & 14-Oct-00 & 6.7 \\
\hline 141.6 & 0.3 & 21.214 & 2000.959 & 16-Dec-00 & 6.7 \\
\hline 141.9 & 1.5 & 21.408 & 2001.153 & $25-$ Feb-01 & 6.6 \\
\hline
\end{tabular}




\section{SF-05 Salvio Street in Concord Concord Fault}

\begin{tabular}{|c|c|c|c|c|c|}
\hline $\begin{array}{l}\text { movement } \\
(\mathrm{mm})\end{array}$ & std. dev. & \# of years & year & date & $\begin{array}{c}\mathrm{mm} / \mathrm{yr}-\mathrm{simpl} \\
\text { average }\end{array}$ \\
\hline 0.0 & 1.5 & 0.000 & 1979.748 & 30-Sep-79 & \\
\hline 8.8 & 1.8 & 0.173 & 1979.921 & 2-Dec-79 & \\
\hline 8.5 & 1.3 & 0.307 & 1980.055 & 20-Jan-80 & \\
\hline 10.0 & 0.9 & 0.326 & 1980.074 & 27-Jan-80 & \\
\hline 8.8 & 1.9 & 0.345 & 1980.093 & 3-Feb-80 & \\
\hline 9.2 & 0.6 & 0.785 & 1980.533 & 13-Jul-80 & \\
\hline 8.7 & 0.6 & 0.957 & 1980.705 & 14-Sep-80 & 9.1 \\
\hline 8.3 & 0.5 & 1.110 & 1980.858 & 9-Nov-80 & 7.5 \\
\hline 8.6 & 1.4 & 1.301 & 1981.049 & 18-Jan-81 & 6.6 \\
\hline 9.8 & 0.5 & 1.359 & 1981.107 & 8-Feb-81 & 7.2 \\
\hline 9.2 & 0.5 & 1.704 & 1981.452 & 14-Jun-81 & 5.4 \\
\hline 9.3 & 0.3 & 1.857 & 1981.605 & 9-Aug-81 & 5.0 \\
\hline 9.6 & 0.6 & 2.184 & 1981.932 & 6-Dec-81 & 4.4 \\
\hline 11.9 & 1.0 & 2.490 & 1982.238 & 28-Mar-82 & 4.8 \\
\hline 12.2 & 1.4 & 2.701 & 1982.449 & 13-Jun-82 & 4.5 \\
\hline 11.9 & 0.6 & 2.816 & 1982.564 & 25-Jul-82 & 4.2 \\
\hline 12.3 & 0.5 & 2.970 & 1982.718 & 19-Sep-82 & 4.1 \\
\hline 10.6 & 0.7 & 3.047 & 1982.795 & 17-Oct-82 & 3.5 \\
\hline 11.6 & 0.7 & 3.200 & 1982.948 & 12-Dec-82 & 3.6 \\
\hline 13.0 & 0.4 & 3.411 & 1983.159 & 27-Feb-83 & 3.8 \\
\hline 12.4 & 0.4 & 3.641 & 1983.389 & 22-May-83 & 3.4 \\
\hline 12.1 & 0.7 & 3.794 & 1983.542 & 17-Jul-83 & 3.2 \\
\hline 11.5 & 0.7 & 4.005 & 1983.753 & $2-0 c t-83$ & 2.9 \\
\hline 11.1 & 0.6 & 4.178 & 1983.926 & 4-Dec-83 & 2.7 \\
\hline 12.3 & 1.2 & 4.331 & 1984.079 & 29-Jan-84 & 2.8 \\
\hline 12.7 & 1.3 & 4.484 & 1984.232 & 25-Mar-84 & 2.8 \\
\hline 13.5 & 1.4 & 4.714 & 1984.462 & 17-Jun-84 & 2.9 \\
\hline 20.0 & 0.7 & 4.905 & 1984.653 & 26-Aug-84 & 4.1 \\
\hline 20.0 & 1.6 & 4.943 & 1984.691 & 9-Sep-84 & 4.0 \\
\hline 20.1 & 0.3 & 5.039 & 1984.787 & $14-0 c t-84$ & 4.0 \\
\hline 20.8 & 0.3 & 5.135 & 1984.883 & 18-Nov-84 & 4.0 \\
\hline 21.2 & 0.3 & 5.307 & 1985.055 & 20-Jan-85 & 4.0 \\
\hline 21.2 & 0.3 & 5.460 & 1985.208 & 17-Mar-85 & 3.9 \\
\hline 20.4 & 0.6 & 5.671 & 1985.419 & 2-Jun-85 & 3.6 \\
\hline 20.8 & 0.2 & 5.786 & 1985.534 & 14-Jul-85 & 3.6 \\
\hline 19.5 & 0.9 & 6.016 & 1985.764 & $6-0 c t-85$ & 3.2 \\
\hline 20.0 & 0.7 & 6.323 & 1986.071 & 26-Jan-86 & 3.2 \\
\hline 22.0 & 0.4 & 6.477 & 1986.225 & 23-Mar-86 & 3.4 \\
\hline 20.8 & 0.6 & 6.707 & 1986.455 & 15-Jun-86 & 3.1 \\
\hline 22.0 & 1.0 & 6.841 & 1986.589 & 3-Aug-86 & 3.2 \\
\hline 20.3 & 0.4 & 7.071 & 1986.819 & $26-0 c t-86$ & 2.9 \\
\hline 20.6 & 0.3 & 7.340 & 1987.088 & 1-Feb-87 & 2.8 \\
\hline 22.4 & 1.2 & 7.531 & 1987.279 & 12-Apr-87 & 3.0 \\
\hline 21.9 & 1.0 & 7.704 & 1987.452 & 14-Jun-87 & 2.8 \\
\hline 21.0 & 0.8 & 7.896 & 1987.644 & 23-Aug-87 & 2.7 \\
\hline 21.3 & 1.1 & 8.164 & 1987.912 & 29-Nov-87 & 2.6 \\
\hline
\end{tabular}




\begin{tabular}{|c|c|c|c|c|c|}
\hline 29.0 & 0.8 & 8.394 & 1988.142 & $21-F e b-88$ & 3.5 \\
\hline 28.8 & 0.5 & 8.471 & 1988.219 & 20-Mar-88 & 3.4 \\
\hline 29.2 & 0.9 & 8.566 & 1988.314 & 24-Apr-88 & 3.4 \\
\hline 30.3 & 0.6 & 8.777 & 1988.525 & 10-Jul-88 & 3.5 \\
\hline 29.7 & 0.9 & 8.949 & 1988.697 & $11-$ Sep-88 & 3.3 \\
\hline 28.9 & 0.5 & 9.121 & 1988.869 & 13-Nov-88 & 3.2 \\
\hline 28.3 & 0.4 & 9.331 & 1989.079 & 29-Jan-89 & 3.0 \\
\hline 31.0 & 0.2 & 9.523 & 1989.271 & 9-Apr-89 & 3.3 \\
\hline 31.5 & 1.2 & 9.715 & 1989.463 & 18-Jun-89 & 3.2 \\
\hline 31.9 & 0.6 & 9.888 & 1989.636 & 20-Aug-89 & 3.2 \\
\hline 33.0 & 1.3 & 10.156 & 1989.904 & 26-Nov-89 & 3.2 \\
\hline 33.8 & 0.8 & 10.348 & 1990.096 & 4-Feb-90 & 3.3 \\
\hline 34.5 & 1.0 & 10.444 & 1990.192 & 11-Mar-90 & 3.3 \\
\hline 35.0 & 0.3 & 10.518 & 1990.266 & 7-Apr-90 & 3.3 \\
\hline 35.9 & 0.3 & 10.578 & 1990.326 & 29-Apr-90 & 3.4 \\
\hline 35.2 & 0.9 & 10.731 & 1990.479 & 24-Jun-90 & 3.3 \\
\hline 33.9 & 1.6 & 10.885 & 1990.633 & 19-Aug-90 & 3.1 \\
\hline 35.0 & 0.9 & 11.019 & 1990.767 & 7-Oct-90 & 3.2 \\
\hline 33.3 & 0.5 & 11.192 & 1990.940 & 9-Dec-90 & 3.0 \\
\hline 34.2 & 1.1 & 11.364 & 1991.112 & 10-Feb-91 & 3.0 \\
\hline 35.8 & 0.9 & 11.518 & 1991.266 & 7-Apr-91 & 3.1 \\
\hline 36.1 & 1.1 & 11.729 & 1991.477 & 23-Jun-91 & 3.1 \\
\hline 35.8 & 1.3 & 11.863 & 1991.611 & 11-Aug-91 & 3.0 \\
\hline 35.2 & 0.7 & 11.997 & 1991.745 & 29-Sep-91 & 2.9 \\
\hline 36.7 & 0.1 & 12.227 & 1991.975 & 22-Dec-91 & 3.0 \\
\hline 35.9 & 0.3 & 12.438 & 1992.186 & 8-Mar-92 & 2.9 \\
\hline 38.1 & 0.9 & 12.591 & 1992.339 & 3-May-92 & 3.0 \\
\hline 38.1 & 0.3 & 12.782 & 1992.530 & 12-Jul-92 & 3.0 \\
\hline 35.2 & 1.3 & 13.031 & 1992.779 & 11-Oct-92 & 2.7 \\
\hline 35.3 & 1.0 & 13.184 & 1992.932 & 6-Dec-92 & 2.7 \\
\hline 41.4 & 1.5 & 13.433 & 1993.181 & 7-Mar-93 & 3.1 \\
\hline 45.5 & 0.8 & 13.625 & 1993.373 & 16-May-93 & 3.3 \\
\hline 44.7 & 0.8 & 13.874 & 1993.622 & 15-Aug-93 & 3.2 \\
\hline 45.3 & 0.6 & 14.027 & 1993.775 & 10-Oct-93 & 3.2 \\
\hline 45.2 & 1.3 & 14.216 & 1993.964 & 18-Dec-93 & 3.2 \\
\hline 45.9 & 2.4 & 14.449 & 1994.197 & 13-Mar-94 & 3.2 \\
\hline 47.0 & 1.1 & 14.622 & 1994.370 & 15-May-94 & 3.2 \\
\hline 45.1 & 1.3 & 14.814 & 1994.562 & 24-Jul-94 & 3.0 \\
\hline 45.5 & 5.4 & 15.003 & 1994.751 & $1-$ Oct-94 & 3.0 \\
\hline 45.5 & 1.0 & 15.194 & 1994.942 & 10-Dec-94 & 3.0 \\
\hline 47.7 & 1.8 & 15.351 & 1995.099 & 5-Feb-95 & 3.1 \\
\hline 47.5 & 0.9 & 15.427 & 1995.175 & 5-Mar-95 & 3.1 \\
\hline 46.6 & 0.5 & 15.715 & 1995.463 & 18-Jun-95 & 3.0 \\
\hline 46.1 & 1.1 & 15.945 & 1995.693 & 10-Sep-95 & 2.9 \\
\hline 45.7 & 1.0 & 16.156 & 1995.904 & 26-Nov-95 & 2.8 \\
\hline 45.9 & 2.3 & 16.288 & 1996.036 & 13-Jan-96 & 2.8 \\
\hline 47.5 & 2.6 & 16.501 & 1996.249 & 31-Mar-96 & 2.9 \\
\hline 47.3 & 2.4 & 16.730 & 1996.478 & 23-Jun-96 & 2.8 \\
\hline 46.8 & 1.5 & 16.902 & 1996.650 & 25-Aug-96 & 2.8 \\
\hline
\end{tabular}




\begin{tabular}{|c|c|c|c|c|c|}
\hline 46.1 & 1.3 & 17.074 & 1996.822 & 27-Oct-96 & 2.7 \\
\hline 54.3 & 1.0 & 17.208 & 1996.956 & 15-Dec-96 & 3.2 \\
\hline 55.3 & 1.0 & 17.457 & 1997.205 & 16-Mar-97 & 3.2 \\
\hline 54.3 & 0.3 & 17.630 & 1997.378 & 18-May-97 & 3.1 \\
\hline 55.3 & 0.5 & 17.764 & 1997.512 & 6-Jul-97 & 3.1 \\
\hline 54.8 & 0.6 & 17.994 & 1997.742 & 28-Sep-97 & 3.0 \\
\hline 55.4 & 0.8 & 18.167 & 1997.915 & 30-Nov-97 & 3.0 \\
\hline 56.0 & 0.4 & 18.301 & 1998.049 & 18-Jan-98 & 3.1 \\
\hline 55.7 & 0.2 & 18.455 & 1998.203 & 15-Mar-98 & 3.0 \\
\hline 57.8 & 0.6 & 18.685 & 1998.433 & 7-Jun-98 & 3.1 \\
\hline 58.0 & 0.6 & 18.838 & 1998.586 & 2-Aug-98 & 3.1 \\
\hline 56.8 & 0.9 & 19.068 & 1998.816 & $25-$ Oct-98 & 3.0 \\
\hline 55.4 & 0.7 & 19.222 & 1998.970 & 20-Dec-98 & 2.9 \\
\hline 57.2 & 0.2 & 19.392 & 1999.140 & 20-Feb-99 & 3.0 \\
\hline 57.6 & 0.3 & 19.510 & 1999.258 & 4-Apr-99 & 3.0 \\
\hline 58.2 & 0.7 & 19.682 & 1999.430 & 6-Jun-99 & 3.0 \\
\hline 58.0 & 1.4 & 19.855 & 1999.603 & 8-Aug-99 & 2.9 \\
\hline 56.5 & 2.4 & 20.036 & 1999.814 & 24-Oct-99 & 2.8 \\
\hline 57.3 & 2.8 & 20.189 & 1999.967 & 19-Dec-99 & 2.8 \\
\hline 57.6 & 2.4 & 20.410 & 2000.158 & 27-Feb-00 & 2.8 \\
\hline 58.2 & 1.6 & 20.563 & 2000.311 & 23-Apr-00 & 2.8 \\
\hline 58.4 & 1.8 & 20.736 & 2000.484 & 25-Jun-00 & 2.8 \\
\hline 56.7 & 0.8 & 20.908 & 2000.656 & 27-Aug-00 & 2.7 \\
\hline 57.7 & 1.8 & 21.042 & 2000.790 & $15-$ Oct-00 & 2.7 \\
\hline 58.8 & 0.9 & 21.195 & 2000.943 & 10-Dec-00 & 2.8 \\
\hline 59.4 & 1.2 & 21.367 & 2001.115 & $11-$ Feb-01 & 2.8 \\
\hline
\end{tabular}


SF-06 Wright Road near Hollister Calaveras Fault

\begin{tabular}{|c|c|c|c|c|c|}
\hline $\begin{array}{l}\text { movement } \\
(\mathrm{mm})\end{array}$ & std. dev. & \# of years & year & date & $\begin{array}{c}\mathrm{mm} / \mathrm{yr} \text {-simple } \\
\text { average }\end{array}$ \\
\hline 0.0 & 1.5 & 0.000 & 1979.805 & 21-Oct-79 & \\
\hline-0.1 & 0.4 & 0.255 & 1980.060 & 22-Jan-80 & \\
\hline 4.3 & 0.6 & 0.362 & 1980.167 & 1-Mar-80 & \\
\hline 3.7 & 0.9 & 0.381 & 1980.186 & 8-Mar-80 & \\
\hline 4.8 & 1.1 & 0.534 & 1980.339 & 3-May-80 & \\
\hline 8.0 & 1.6 & 0.668 & 1980.473 & 21-Jun-80 & 12.0 \\
\hline 19.3 & 1.2 & 1.242 & 1981.047 & 17-Jan-81 & 15.5 \\
\hline 20.3 & 0.5 & 1.663 & 1981.468 & 20-Jun-81 & 12.2 \\
\hline 30.7 & 0.5 & 1.836 & 1981.641 & 22-Aug-81 & 16.7 \\
\hline 31.6 & 0.6 & 2.143 & 1981.948 & 12-Dec-81 & 14.7 \\
\hline 31.7 & 0.1 & 2.507 & 1982.312 & 24-Apr-82 & 12.6 \\
\hline 38.8 & 0.3 & 2.776 & 1982.581 & 31-Jul-82 & 14.0 \\
\hline 45.2 & 0.5 & 3.102 & 1982.907 & 27-Nov-82 & 14.6 \\
\hline 45.8 & 0.6 & 3.351 & 1983.156 & 26-Feb-83 & 13.7 \\
\hline 51.5 & 0.2 & 3.548 & 1983.353 & 9-May-83 & 14.5 \\
\hline 51.9 & 0.1 & 3.642 & 1983.447 & 12-Jun-83 & 14.3 \\
\hline 52.8 & 0.2 & 3.781 & 1983.586 & 2-Aug-83 & 14.0 \\
\hline 53.9 & 0.5 & 4.003 & 1983.808 & $22-0 c t-83$ & 13.5 \\
\hline 58.2 & 0.4 & 4.272 & 1984.077 & 28-Jan-84 & 13.6 \\
\hline 59.2 & 0.4 & 4.512 & 1984.317 & 25-Apr-84 & 13.1 \\
\hline 59.6 & 0.5 & 4.616 & 1984.421 & 2-Jun-84 & 12.9 \\
\hline 70.0 & 0.7 & 4.673 & 1984.478 & 23-Jun-84 & 15.0 \\
\hline 66.3 & 0.3 & 4.714 & 1984.519 & 8-Jul-84 & 14.1 \\
\hline 72.5 & 0.4 & 4.804 & 1984.609 & 10-Aug-84 & 15.1 \\
\hline 72.2 & 0.4 & 4.963 & 1984.768 & $7-$-Oct-84 & 14.5 \\
\hline 72.6 & 0.7 & 5.132 & 1984.937 & 8-Dec-84 & 14.1 \\
\hline 72.0 & 0.6 & 5.266 & 1985.071 & 26-Jan-85 & 13.7 \\
\hline 76.2 & 0.8 & 5.458 & 1985.263 & 6-Apr-85 & 14.0 \\
\hline 83.4 & 0.3 & 5.688 & 1985.493 & 29-Jun-85 & 14.7 \\
\hline 86.8 & 0.4 & 5.940 & 1985.745 & 29-Sep-85 & 14.6 \\
\hline 86.4 & 0.1 & 6.129 & 1985.934 & 7-Dec-85 & 14.1 \\
\hline 86.9 & 0.7 & 6.274 & 1986.079 & 29-Jan-86 & 13.9 \\
\hline 87.1 & 0.8 & 6.321 & 1986.126 & 15-Feb-86 & 13.8 \\
\hline 86.7 & 0.4 & 6.532 & 1986.337 & 3-May-86 & 13.3 \\
\hline 87.7 & 0.5 & 6.622 & 1986.427 & 5-Jun-86 & 13.2 \\
\hline 86.7 & 0.7 & 6.800 & 1986.605 & 9-Aug-86 & 12.8 \\
\hline 87.3 & 0.4 & 6.973 & 1986.778 & 11-Oct-86 & 12.5 \\
\hline 88.3 & 0.9 & 7.127 & 1986.932 & 6-Dec-86 & 12.4 \\
\hline 94.2 & 0.8 & 7.357 & 1987.162 & 28-Feb-87 & 12.8 \\
\hline 94.5 & 1.2 & 7.609 & 1987.414 & 31-May-87 & 12.4 \\
\hline 99.7 & 0.8 & 7.724 & 1987.529 & 12-Jul-87 & 12.9 \\
\hline 100.8 & 0.6 & 7.817 & 1987.622 & 15-Aug-87 & 12.9 \\
\hline 100.2 & 1.0 & 8.085 & 1987.890 & 21-Nov-87 & 12.4 \\
\hline 103.9 & 0.3 & 8.277 & 1988.082 & 30-Jan-88 & 12.6 \\
\hline 104.4 & 0.7 & 8.356 & 1988.161 & 28-Feb-88 & 12.5 \\
\hline 103.6 & 0.4 & 8.526 & 1988.331 & 30-Apr-88 & 12.2 \\
\hline
\end{tabular}




\begin{tabular}{|c|c|c|c|c|c|}
\hline 105.1 & 0.6 & 8.649 & 1988.454 & 14-Jun-88 & 12.2 \\
\hline 104.2 & 0.6 & 8.717 & 1988.522 & 9-Jul-88 & 12.0 \\
\hline 111.8 & 0.2 & 8.949 & 1988.754 & 2-Oct-88 & 12.5 \\
\hline 116.0 & 0.4 & 9.118 & 1988.923 & 3-Dec-88 & 12.7 \\
\hline 116.2 & 0.6 & 9.310 & 1989.115 & 11-Feb-89 & 12.5 \\
\hline 116.4 & 0.6 & 9.463 & 1989.268 & 8-Apr-89 & 12.3 \\
\hline 115.9 & 0.4 & 9.655 & 1989.460 & 17-Jun-89 & 12.0 \\
\hline 119.2 & 0.2 & 9.828 & 1989.633 & 19-Aug-89 & 12.1 \\
\hline 131.4 & 0.1 & 10.000 & 1989.805 & 21-Oct-89 & 13.1 \\
\hline 132.1 & 0.6 & 10.058 & 1989.863 & 11-Nov-89 & 13.1 \\
\hline 132.0 & 0.4 & 10.135 & 1989.940 & 9-Dec-89 & 13.0 \\
\hline 131.8 & 0.4 & 10.231 & 1990.036 & 13-Jan-90 & 12.9 \\
\hline 132.5 & 0.4 & 10.346 & 1990.151 & 24-Feb-90 & 12.8 \\
\hline 131.4 & 0.4 & 10.480 & 1990.285 & 14-Apr-90 & 12.5 \\
\hline 131.7 & 0.3 & 10.576 & 1990.381 & 19-May-90 & 12.5 \\
\hline 132.9 & 0.3 & 10.713 & 1990.518 & 8-Jul-90 & 12.4 \\
\hline 132.5 & 0.5 & 10.809 & 1990.614 & 12-Aug-90 & 12.3 \\
\hline 133.1 & 0.3 & 10.924 & 1990.729 & 23-Sep-90 & 12.2 \\
\hline 133.6 & 0.3 & 11.036 & 1990.841 & 3-Nov-90 & 12.1 \\
\hline 132.9 & 0.5 & 11.266 & 1991.071 & 26-Jan-91 & 11.8 \\
\hline 132.9 & 0.2 & 11.458 & 1991.263 & 6-Apr-91 & 11.6 \\
\hline 133.1 & 0.4 & 11.631 & 1991.436 & 8-Jun-91 & 11.4 \\
\hline 132.2 & 0.6 & 11.880 & 1991.685 & 7-Sep-91 & 11.1 \\
\hline 133.0 & 0.5 & 12.072 & 1991.877 & 16-Nov-91 & 11.0 \\
\hline 132.9 & 0.4 & 12.250 & 1992.055 & 20-Jan-92 & 10.8 \\
\hline 131.3 & 0.6 & 12.474 & 1992.279 & 11-Apr-92 & 10.5 \\
\hline 132.6 & 0.5 & 12.665 & 1992.470 & 20-Jun-92 & 10.5 \\
\hline 132.4 & 2.0 & 12.821 & 1992.626 & 16-Aug-92 & 10.3 \\
\hline 132.4 & 0.2 & 12.993 & 1992.798 & 18-Oct-92 & 10.2 \\
\hline 130.7 & 0.6 & 13.165 & 1992.970 & 20-Dec-92 & 9.9 \\
\hline 131.1 & 0.9 & 13.239 & 1993.044 & 16-Jan-93 & 9.9 \\
\hline 132.9 & 1.3 & 13.392 & 1993.197 & 13-Mar-93 & 9.9 \\
\hline 131.3 & 1.3 & 13.548 & 1993.353 & 9-Мау-93 & 9.7 \\
\hline 131.2 & 0.7 & 13.757 & 1993.562 & 24-Jul-93 & 9.5 \\
\hline 140.1 & 0.8 & 13.853 & 1993.658 & 28-Aug-93 & 10.1 \\
\hline 140.3 & 1.6 & 14.025 & 1993.830 & 30-Oct-93 & 10.0 \\
\hline 141.2 & 1.1 & 14.236 & 1994.041 & 15-Jan-94 & 9.9 \\
\hline 141.9 & 0.6 & 14.428 & 1994.233 & 26-Mar-94 & 9.8 \\
\hline 144.0 & 0.5 & 14.620 & 1994.425 & 4-Jun-94 & 9.8 \\
\hline 147.3 & 1.4 & 14.798 & 1994.603 & 8-Aug-94 & 10.0 \\
\hline 149.3 & 1.0 & 15.061 & 1994.866 & 12-Nov-94 & 9.9 \\
\hline 147.5 & 0.9 & 15.255 & 1995.060 & 22-Jan-95 & 9.7 \\
\hline 149.1 & 1.0 & 15.310 & 1995.115 & 11-Feb-95 & 9.7 \\
\hline 150.9 & 1.6 & 15.502 & 1995.307 & 22-Apr-95 & 9.7 \\
\hline 148.2 & 0.6 & 15.524 & 1995.329 & 30-Apr-95 & 9.5 \\
\hline 156.0 & 0.8 & 15.732 & 1995.537 & 15-Jul-95 & 9.9 \\
\hline 160.2 & 1.3 & 15.965 & 1995.770 & 8-Oct-95 & 10.0 \\
\hline 162.8 & 0.8 & 16.192 & 1995.997 & 30-Dec-95 & 10.1 \\
\hline 166.5 & 2.6 & 16.384 & 1996.189 & 9-Mar-96 & 10.2 \\
\hline
\end{tabular}




\begin{tabular}{|c|c|c|c|c|c|}
\hline 163.8 & 0.7 & 16.520 & 1996.325 & 28-Apr-96 & 9.9 \\
\hline 165.8 & 3.7 & 16.594 & 1996.399 & 25-May-96 & 10.0 \\
\hline 166.4 & 0.8 & 16.766 & 1996.571 & 27-Jul-96 & 9.9 \\
\hline 167.5 & 1.0 & 16.938 & 1996.743 & 28-Sep-96 & 9.9 \\
\hline 172.6 & 0.7 & 17.072 & 1996.877 & 16-Nov-96 & 10.1 \\
\hline 172.8 & 1.5 & 17.244 & 1997.049 & 18-Jan-97 & 10.0 \\
\hline 175.0 & 1.0 & 17.417 & 1997.222 & 22-Mar-97 & 10.0 \\
\hline 174.1 & 0.4 & 17.551 & 1997.356 & 10-May-97 & 9.9 \\
\hline 175.7 & 1.2 & 17.724 & 1997.529 & 12-Jul-97 & 9.9 \\
\hline 185.4 & 0.6 & 17.918 & 1997.723 & 21-Sep-97 & 10.3 \\
\hline 186.0 & 0.3 & 18.107 & 1997.912 & 29-Nov-97 & 10.3 \\
\hline 185.0 & 0.7 & 18.280 & 1998.085 & 31-Jan-98 & 10.1 \\
\hline 187.3 & 0.7 & 18.436 & 1998.241 & 29-Mar-98 & 10.2 \\
\hline 187.4 & 0.7 & 18.606 & 1998.411 & 30-May-98 & 10.1 \\
\hline 187.2 & 0.7 & 18.759 & 1998.564 & 25-Jul-98 & 10.0 \\
\hline 186.8 & 0.6 & 18.817 & 1998.622 & 15-Aug-98 & 9.9 \\
\hline 195.7 & 0.4 & 19.028 & 1998.833 & 31-Oct-98 & 10.3 \\
\hline 196.7 & 1.0 & 19.220 & 1999.025 & 9-Jan-99 & 10.2 \\
\hline 198.2 & 1.4 & 19.376 & 1999.181 & 7-Mar-99 & 10.2 \\
\hline 197.1 & 0.8 & 19.546 & 1999.351 & 8-May-99 & 10.1 \\
\hline 197.3 & 0.6 & 19.718 & 1999.523 & 10-Jul-99 & 10.0 \\
\hline 196.9 & 0.9 & 19.929 & 1999.734 & 25-Sep-99 & 9.9 \\
\hline 202.2 & 0.7 & 20.102 & 1999.907 & 27-Nov-99 & 10.1 \\
\hline 203.4 & 0.7 & 20.293 & 2000.098 & 5-Feb-00 & 10.0 \\
\hline 209.1 & 0.6 & 20.465 & 2000.270 & 8-Apr-00 & 10.2 \\
\hline 208.0 & 0.8 & 20.638 & 2000.443 & 10-Jun-00 & 10.1 \\
\hline 205.3 & 0.8 & 20.829 & 2000.634 & 19-Aug-00 & 9.9 \\
\hline 207.2 & 1.3 & 20.982 & 2000.787 & 14-Oct-00 & 9.9 \\
\hline 206.9 & 1.0 & 21.154 & 2000.959 & 16-Dec-00 & 9.8 \\
\hline 211.1 & 0.4 & 21.348 & 2001.153 & 25-Feb-01 & 9.9 \\
\hline
\end{tabular}


SF-07 West Point Ave in Princeton San Gregorio (Seal Cove) Fault

\begin{tabular}{|c|c|c|c|c|c|}
\hline $\begin{array}{l}\text { movement } \\
(\mathrm{mm})\end{array}$ & std. dev. & \# of years & year & date & $\begin{array}{c}\mathrm{mm} / \mathrm{yr} \text {-simple } \\
\text { average }\end{array}$ \\
\hline 0.0 & 3.4 & 0.000 & 1979.858 & 9-Nov-79 & \\
\hline-3.9 & 1.8 & 0.290 & 1980.148 & 23-Feb-80 & \\
\hline-3.0 & 1.8 & 0.500 & 1980.358 & 10-May-80 & \\
\hline-0.3 & 1.5 & 0.729 & 1980.587 & 2-Aug-80 & \\
\hline 1.6 & 1.1 & 0.869 & 1980.727 & $22-$ Sep-80 & 1.8 \\
\hline-0.3 & 2.8 & 1.038 & 1980.896 & 23-Nov-80 & -0.3 \\
\hline 2.1 & 1.2 & 1.200 & 1981.058 & 20-Jan-81 & 1.8 \\
\hline 6.0 & 0.5 & 1.394 & 1981.252 & 2-Apr-81 & 4.3 \\
\hline 6.0 & 1.7 & 1.660 & 1981.518 & 8-Jul-81 & 3.6 \\
\hline 6.7 & 0.9 & 1.745 & 1981.603 & 8-Aug-81 & 3.8 \\
\hline 4.8 & 1.4 & 1.975 & 1981.833 & $31-$-ct-81 & 2.4 \\
\hline 8.5 & 0.6 & 2.216 & 1982.074 & 27-Jan-82 & 3.8 \\
\hline 7.9 & 0.8 & 2.526 & 1982.384 & 20-May-82 & 3.1 \\
\hline 6.8 & 0.8 & 2.712 & 1982.570 & 27-Jul-82 & 2.5 \\
\hline 5.1 & 1.2 & 3.087 & 1982.945 & 11-Dec-82 & 1.7 \\
\hline 5.3 & 0.9 & 3.416 & 1983.274 & 10-Apr-83 & 1.6 \\
\hline 3.8 & 1.6 & 3.597 & 1983.455 & 15-Jun-83 & 1.1 \\
\hline 1.3 & 0.8 & 3.739 & 1983.597 & 6-Aug-83 & 0.3 \\
\hline 4.6 & 1.1 & 3.912 & 1983.770 & 8-Oct-83 & 1.2 \\
\hline 3.5 & 1.7 & 4.221 & 1984.079 & 29-Jan-84 & 0.8 \\
\hline 4.4 & 1.1 & 4.538 & 1984.396 & 24-May-84 & 1.0 \\
\hline 2.3 & 1.2 & 4.639 & 1984.497 & 30-Jun-84 & 0.5 \\
\hline 2.9 & 1.1 & 4.964 & 1984.822 & $27-O c t-84$ & 0.6 \\
\hline 5.3 & 0.3 & 5.257 & 1985.115 & 11-Feb-85 & 1.0 \\
\hline 2.7 & 0.2 & 5.487 & 1985.345 & 6-May-85 & 0.5 \\
\hline 5.8 & 0.1 & 5.602 & 1985.460 & 17-Jun-85 & 1.0 \\
\hline 6.8 & 0.9 & 5.731 & 1985.589 & 3-Aug-85 & 1.2 \\
\hline 3.0 & 1.1 & 6.098 & 1985.956 & 15-Dec-85 & 0.5 \\
\hline 3.2 & 0.7 & 6.249 & 1986.107 & 8-Feb-86 & 0.5 \\
\hline 1.5 & 0.4 & 6.421 & 1986.279 & 12-Apr-86 & 0.2 \\
\hline 4.1 & 1.3 & 6.589 & 1986.447 & 12-Jun-86 & 0.6 \\
\hline 4.4 & 0.8 & 6.901 & 1986.759 & $4-O c t-86$ & 0.6 \\
\hline 3.9 & 1.1 & 7.227 & 1987.085 & 31-Jan-87 & 0.5 \\
\hline 6.3 & 1.2 & 7.419 & 1987.277 & 11-Apr-87 & 0.8 \\
\hline 3.0 & 1.3 & 7.783 & 1987.641 & 22-Aug-87 & 0.4 \\
\hline 3.0 & 2.0 & 8.013 & 1987.871 & 14-Nov-87 & 0.4 \\
\hline 4.9 & 1.1 & 8.262 & 1988.120 & 13-Feb-88 & 0.6 \\
\hline 3.7 & 1.0 & 8.533 & 1988.391 & 22-May-88 & 0.4 \\
\hline 2.3 & 1.4 & 8.893 & 1988.751 & 1-Oct-88 & 0.3 \\
\hline 4.3 & 1.0 & 9.106 & 1988.964 & 18-Dec-88 & 0.5 \\
\hline 4.2 & 1.7 & 9.679 & 1989.537 & 15-Jul-89 & 0.4 \\
\hline 2.2 & 1.3 & 9.967 & 1989.825 & $28-0 c t-89$ & 0.2 \\
\hline 5.9 & 1.1 & 10.216 & 1990.074 & 27-Jan-90 & 0.6 \\
\hline 5.9 & 2.1 & 10.372 & 1990.230 & $25-$ Mar-90 & 0.6 \\
\hline 0.3 & 0.8 & 10.580 & 1990.438 & 9-Jun-90 & 0.0 \\
\hline 1.2 & 1.7 & 10.791 & 1990.649 & 25-Aug-90 & 0.1 \\
\hline
\end{tabular}




\begin{tabular}{|c|c|c|c|c|c|}
\hline-5.7 & 3.6 & 11.002 & 1990.860 & 10-Nov-90 & -0.5 \\
\hline 4.2 & 2.1 & 11.293 & 1991.151 & 24-Feb-91 & 0.4 \\
\hline 9.4 & 3.5 & 11.424 & 1991.282 & 13-Apr-91 & 0.8 \\
\hline 5.1 & 2.1 & 11.600 & 1991.458 & 16-Jun-91 & 0.4 \\
\hline 1.2 & 1.6 & 11.791 & 1991.649 & 25-Aug-91 & 0.1 \\
\hline 0.0 & 1.9 & 11.846 & 1991.704 & 14-Sep-91 & 0.0 \\
\hline-0.3 & 1.9 & 12.076 & 1991.934 & 7-Dec-91 & 0.0 \\
\hline 7.5 & 0.9 & 12.347 & 1992.205 & 15-Mar-92 & 0.6 \\
\hline 2.7 & 2.3 & 12.653 & 1992.511 & 5-Jul-92 & 0.2 \\
\hline-9.3 & 1.7 & 12.956 & 1992.814 & 24-Oct-92 & -0.7 \\
\hline 3.9 & 1.3 & 13.243 & 1993.101 & 6-Feb-93 & 0.3 \\
\hline 2.2 & 1.8 & 13.610 & 1993.468 & 20-Jun-93 & 0.2 \\
\hline-5.2 & 1.7 & 13.841 & 1993.699 & 12-Sep-93 & -0.4 \\
\hline 4.3 & 1.3 & 14.241 & 1994.099 & 5-Feb-94 & 0.3 \\
\hline 3.8 & 2.1 & 14.509 & 1994.367 & 14-May-94 & 0.3 \\
\hline-1.0 & 1.0 & 14.750 & 1994.608 & 10-Aug-94 & -0.1 \\
\hline 1.7 & 1.4 & 15.112 & 1994.970 & 20-Dec-94 & 0.1 \\
\hline 3.3 & 1.2 & 15.375 & 1995.233 & 26-Mar-95 & 0.2 \\
\hline-2.8 & 3.2 & 15.621 & 1995.479 & 24-Jun-95 & -0.2 \\
\hline-2.8 & 2.1 & 15.947 & 1995.805 & 21-Oct-95 & -0.2 \\
\hline 6.2 & 1.3 & 16.199 & 1996.057 & 21-Jan-96 & 0.4 \\
\hline 4.1 & 1.5 & 16.484 & 1996.342 & 4-May-96 & 0.2 \\
\hline 2.5 & 3.1 & 16.735 & 1996.593 & 4-Aug-96 & 0.1 \\
\hline 3.1 & 0.5 & 17.038 & 1996.896 & 23-Nov-96 & 0.2 \\
\hline 5.0 & 0.4 & 17.287 & 1997.145 & 22-Feb-97 & 0.3 \\
\hline 1.0 & 1.5 & 17.537 & 1997.395 & 24-May-97 & 0.1 \\
\hline-0.7 & 0.8 & 17.824 & 1997.682 & 6-Sep-97 & 0.0 \\
\hline 3.9 & 1.8 & 18.076 & 1997.934 & 7-Dec-97 & 0.2 \\
\hline 3.7 & 2.8 & 18.361 & 1998.219 & 21-Mar-98 & 0.2 \\
\hline 3.9 & 2.7 & 18.591 & 1998.449 & 13-Jun-98 & 0.2 \\
\hline 6.6 & 2.8 & 18.843 & 1998.701 & 13-Sep-98 & 0.4 \\
\hline 6.4 & 3.3 & 19.191 & 1999.049 & 18-Jan-99 & 0.3 \\
\hline 5.4 & 0.6 & 19.416 & 1999.274 & 10-Apr-99 & 0.3 \\
\hline 0.8 & 2.2 & 19.668 & 1999.526 & 11-Jul-99 & 0.0 \\
\hline-4.0 & 3.6 & 19.915 & 1999.773 & 9-Oct-99 & -0.2 \\
\hline 5.3 & 1.5 & 20.164 & 2000.022 & 8-Jan-00 & 0.3 \\
\hline 0.7 & 0.9 & 20.415 & 2000.273 & 9-Apr-00 & 0.0 \\
\hline 2.3 & 1.1 & 20.683 & 2000.541 & 16-Jul-00 & 0.1 \\
\hline-1.1 & 1.2 & 21.025 & 2000.883 & 18-Nov-00 & -0.1 \\
\hline
\end{tabular}




\section{SF-08 Pescadero Road near Pescadero San Gregorio Fault}

\begin{tabular}{|c|c|c|c|c|c|}
\hline $\begin{array}{l}\text { movement } \\
(\mathrm{mm})\end{array}$ & std. dev. & \# of years & year & date & $\begin{array}{c}\mathrm{mm} / \mathrm{yr} \text {-simple } \\
\text { average }\end{array}$ \\
\hline 0.0 & 0.7 & 0.000 & 1982.384 & 20-May-82 & \\
\hline-4.3 & 1.0 & 0.186 & 1982.570 & 27-Jul-82 & \\
\hline 5.0 & 1.0 & 0.561 & 1982.945 & 11-Dec-82 & \\
\hline 4.4 & 4.2 & 1.684 & 1984.068 & 25-Jan-84 & 2.6 \\
\hline 4.4 & 2.9 & 2.012 & 1984.396 & 24-May-84 & 2.2 \\
\hline 0.0 & 2.2 & 2.113 & 1984.497 & 30-Jun-84 & 0.0 \\
\hline 14.6 & 2.2 & 2.668 & 1985.052 & 19-Jan-85 & 5.5 \\
\hline-8.4 & 1.5 & 2.901 & 1985.285 & 14-Apr-85 & -2.9 \\
\hline 2.4 & 0.8 & 3.052 & 1985.436 & 8-Jun-85 & 0.8 \\
\hline 7.6 & 4.0 & 3.205 & 1985.589 & 3-Aug-85 & 2.4 \\
\hline 0.9 & 2.2 & 3.572 & 1985.956 & 15-Dec-85 & 0.3 \\
\hline 7.6 & 2.0 & 3.723 & 1986.107 & 8-Feb-86 & 2.0 \\
\hline 4.8 & 2.5 & 4.063 & 1986.447 & 12-Jun-86 & 1.2 \\
\hline 4.8 & 1.0 & 4.701 & 1987.085 & 31-Jan-87 & 1.0 \\
\hline 9.4 & 1.2 & 4.893 & 1987.277 & 11-Apr-87 & 1.9 \\
\hline-5.5 & 4.2 & 5.257 & 1987.641 & 22-Aug-87 & -1.0 \\
\hline-3.5 & 2.6 & 5.487 & 1987.871 & 14-Nov-87 & -0.6 \\
\hline 0.6 & 1.9 & 5.736 & 1988.120 & 13-Feb-88 & 0.1 \\
\hline-1.6 & 2.3 & 6.007 & 1988.391 & 22-May-88 & -0.3 \\
\hline-3.6 & 2.0 & 6.367 & 1988.751 & 1-Oct-88 & -0.6 \\
\hline-4.6 & 4.3 & 6.580 & 1988.964 & 18-Dec-88 & -0.7 \\
\hline-6.5 & 3.8 & 7.153 & 1989.537 & 15-Jul-89 & -0.9 \\
\hline-2.1 & 1.8 & 7.441 & 1989.825 & $28-0 c t-89$ & -0.3 \\
\hline 2.7 & 2.2 & 7.690 & 1990.074 & 27-Jan-90 & 0.4 \\
\hline-0.9 & 4.6 & 7.824 & 1990.208 & 17-Mar-90 & -0.1 \\
\hline 0.0 & 2.0 & 8.054 & 1990.438 & 9-Jun-90 & 0.0 \\
\hline-5.0 & 1.3 & 8.265 & 1990.649 & 25-Aug-90 & -0.6 \\
\hline-0.2 & 1.2 & 8.476 & 1990.860 & 10-Nov-90 & 0.0 \\
\hline-4.2 & 2.7 & 8.767 & 1991.151 & 24-Feb-91 & -0.5 \\
\hline-2.7 & 3.6 & 8.898 & 1991.282 & 13-Apr-91 & -0.3 \\
\hline 2.3 & 2.6 & 9.074 & 1991.458 & 16-Jun-91 & 0.3 \\
\hline 4.6 & 3.1 & 9.265 & 1991.649 & 25-Aug-91 & 0.5 \\
\hline 5.0 & 3.7 & 9.320 & 1991.704 & 14-Sep-91 & 0.5 \\
\hline 6.9 & 3.4 & 9.550 & 1991.934 & 7-Dec-91 & 0.7 \\
\hline 10.2 & 3.5 & 9.936 & 1992.320 & 26-Apr-92 & 1.0 \\
\hline 10.9 & 5.4 & 10.127 & 1992.511 & 5-Jul-92 & 1.1 \\
\hline 3.1 & 3.9 & 10.430 & 1992.814 & 24-Oct-92 & 0.3 \\
\hline 6.4 & 1.5 & 10.717 & 1993.101 & 6-Feb-93 & 0.6 \\
\hline 6.4 & 2.4 & 11.315 & 1993.699 & 12-Sep-93 & 0.6 \\
\hline 3.1 & 1.2 & 11.715 & 1994.099 & 5-Feb-94 & 0.3 \\
\hline 7.8 & 1.6 & 11.983 & 1994.367 & 14-May-94 & 0.7 \\
\hline 9.9 & 1.5 & 12.224 & 1994.608 & 10-Aug-94 & 0.8 \\
\hline 6.3 & 1.2 & 12.586 & 1994.970 & 20-Dec-94 & 0.5 \\
\hline 14.2 & 1.3 & 12.849 & 1995.233 & $26-M a r-95$ & 1.1 \\
\hline 12.2 & 1.3 & 13.095 & 1995.479 & 24-Jun-95 & 0.9 \\
\hline 12.6 & 2.1 & 13.421 & 1995.805 & $21-$ Oct-95 & 0.9 \\
\hline
\end{tabular}




$\begin{array}{llllll}11.0 & 1.8 & 13.673 & 1996.057 & \text { 21-Jan-96 } & 0.8 \\ 12.7 & 1.5 & 13.958 & 1996.342 & 4-\text { May-96 } & 0.9 \\ 15.4 & 1.6 & 14.209 & 1996.593 & \text { 4-Aug-96 } & 1.1 \\ 10.9 & 1.0 & 14.512 & 1996.896 & 23-\text { Nov-96 } & 0.7 \\ 10.0 & 1.2 & 14.761 & 1997.145 & 22-\text { Feb-97 } & 0.7 \\ 16.9 & 2.6 & 15.011 & 1997.395 & 24-\text { May-97 } & 1.1 \\ 14.0 & 3.4 & 15.298 & 1997.682 & 6 \text {-Sep-97 } & 0.9 \\ 10.4 & 2.4 & 15.550 & 1997.934 & \text { 7-Dec-97 } & 0.7 \\ 12.5 & 0.6 & 15.835 & 1998.219 & 21 \text {-Mar-98 } & 0.8 \\ 15.6 & 0.7 & 16.065 & 1998.449 & 13-J u n-98 & 1.0 \\ 20.8 & 2.3 & 16.317 & 1998.701 & 13-\text { Sep-98 } & 1.3 \\ 14.7 & 1.9 & 16.665 & 1999.049 & 18-J a n-99 & 0.9 \\ 17.5 & 1.5 & 16.890 & 1999.274 & 10-\text { Apr-99 } & 1.0 \\ 12.1 & 2.8 & 17.142 & 1999.526 & 11-J u l-99 & 0.7 \\ 14.2 & 1.6 & 17.389 & 1999.773 & \text { 9-Oct-99 } & 0.8 \\ 7.6 & 1.1 & 17.638 & 2000.022 & \text { 8-Jan-00 } & 0.4 \\ 8.3 & 2.4 & 17.889 & 2000.273 & \text { 9-Apr-00 } & 0.5 \\ 8.2 & 1.0 & 18.157 & 2000.541 & 16-J u l-00 & 0.5 \\ 7.7 & 0.9 & 18.499 & 2000.883 & \text { 18-Nov-00 } & 0.4\end{array}$




\section{SF-09 Deer Valley Road near Antioch "Antioch" Fault}

\begin{tabular}{|c|c|c|c|c|c|}
\hline $\begin{array}{l}\text { movement } \\
(\mathrm{mm})\end{array}$ & std. dev. & \# of years & year & date & $\begin{array}{c}\mathrm{mm} / \mathrm{yr} \text {-simple } \\
\text { average }\end{array}$ \\
\hline 0.0 & 3.2 & 0.000 & 1982.890 & 21-Nov-82 & \\
\hline-7.6 & 3.1 & 0.439 & 1983.329 & 30-Apr-83 & \\
\hline-0.2 & 4.4 & 0.548 & 1983.438 & 9-Jun-83 & \\
\hline-5.2 & 3.8 & 0.683 & 1983.573 & 28-Jul-83 & \\
\hline-5.4 & 1.8 & 0.959 & 1983.849 & 6-Nov-83 & -5.6 \\
\hline-5.5 & 2.7 & 1.173 & 1984.063 & 23-Jan-84 & -4.7 \\
\hline 1.9 & 5.6 & 1.473 & 1984.363 & 12-May-84 & 1.3 \\
\hline 1.7 & 1.9 & 1.569 & 1984.459 & 16-Jun-84 & 1.1 \\
\hline 2.3 & 1.4 & 1.840 & 1984.730 & 23-Sep-84 & 1.2 \\
\hline 1.7 & 1.5 & 1.990 & 1984.880 & 17-Nov-84 & 0.9 \\
\hline 3.3 & 0.7 & 2.222 & 1985.112 & 10-Feb-85 & 1.5 \\
\hline 6.5 & 1.1 & 2.452 & 1985.342 & 5-May-85 & 2.7 \\
\hline 5.2 & 1.2 & 2.606 & 1985.496 & 30-Jun-85 & 2.0 \\
\hline 4.2 & 2.4 & 2.702 & 1985.592 & 4-Aug-85 & 1.6 \\
\hline 4.2 & 2.3 & 2.973 & 1985.863 & 11-Nov-85 & 1.4 \\
\hline 1.3 & 6.7 & 3.198 & 1986.088 & 1-Feb-86 & 0.4 \\
\hline-0.8 & 2.6 & 3.392 & 1986.282 & 13-Apr-86 & -0.2 \\
\hline 0.2 & 1.5 & 3.554 & 1986.444 & 11-Jun-86 & 0.1 \\
\hline 4.2 & 5.4 & 3.910 & 1986.800 & 19-Oct-86 & 1.1 \\
\hline 1.8 & 2.3 & 4.252 & 1987.142 & 21-Feb-87 & 0.4 \\
\hline 4.4 & 5.6 & 4.655 & 1987.545 & 18-Jul-87 & 0.9 \\
\hline 7.8 & 4.8 & 4.926 & 1987.816 & $25-$ Oct- 87 & 1.6 \\
\hline 7.6 & 2.5 & 5.211 & 1988.101 & $6-F e b-88$ & 1.5 \\
\hline 8.6 & 4.0 & 5.539 & 1988.429 & 5-Jun-88 & 1.6 \\
\hline 9.6 & 2.8 & 5.823 & 1988.713 & 17-Sep-88 & 1.6 \\
\hline 10.7 & 3.9 & 6.072 & 1988.962 & 17-Dec-88 & 1.8 \\
\hline 6.3 & 3.5 & 6.324 & 1989.214 & 19-Mar-89 & 1.0 \\
\hline 9.9 & 4.5 & 6.631 & 1989.521 & 9-Jul-89 & 1.5 \\
\hline 3.3 & 2.7 & 6.877 & 1989.767 & $7-0 c t-89$ & 0.5 \\
\hline 11.5 & 4.2 & 7.159 & 1990.049 & 18-Jan-90 & 1.6 \\
\hline 10.3 & 3.3 & 7.263 & 1990.153 & 25-Feb-90 & 1.4 \\
\hline 4.6 & 1.8 & 7.436 & 1990.326 & 29-Apr-90 & 0.6 \\
\hline 8.8 & 2.4 & 7.609 & 1990.499 & 1-Jul-90 & 1.2 \\
\hline
\end{tabular}




\section{SF-10 Duhallow Way in South San Francisco San Andreas Fault}

\begin{tabular}{|c|c|c|c|c|c|}
\hline $\begin{array}{l}\text { movement } \\
\quad(\mathrm{mm})\end{array}$ & std. dev. & \# of years & year & date & $\begin{array}{c}\mathrm{mm} / \mathrm{yr} \text {-simple } \\
\text { average }\end{array}$ \\
\hline 0.0 & 1.5 & 0.000 & 1980.227 & 23-Mar-80 & \\
\hline 0.5 & 1.4 & 0.273 & 1980.500 & 1-Jul-80 & \\
\hline 2.2 & 2.1 & 0.505 & 1980.732 & 24-Sep-80 & \\
\hline 1.4 & 2.0 & 0.571 & 1980.798 & 18-Oct-80 & \\
\hline 1.3 & 1.0 & 0.664 & 1980.891 & 21-Nov-80 & \\
\hline 3.1 & 1.2 & 0.768 & 1980.995 & 29-Dec-80 & \\
\hline 1.8 & 0.8 & 0.828 & 1981.055 & 20-Jan-81 & \\
\hline 2.9 & 0.4 & 0.981 & 1981.208 & 17-Mar-81 & \\
\hline 2.2 & 0.7 & 1.061 & 1981.288 & 15-Apr-81 & 2.1 \\
\hline 1.6 & 1.0 & 1.299 & 1981.526 & 11-Jul-81 & 1.2 \\
\hline 1.7 & 1.2 & 1.376 & 1981.603 & 8-Aug-81 & 1.2 \\
\hline 2.7 & 1.6 & 1.606 & 1981.833 & $31-$ Oct-81 & 1.7 \\
\hline 2.7 & 1.0 & 1.743 & 1981.970 & 20-Dec-81 & 1.5 \\
\hline 1.5 & 0.9 & 1.863 & 1982.090 & 2-Feb-82 & 0.8 \\
\hline 2.4 & 1.9 & 1.992 & 1982.219 & 21-Mar-82 & 1.2 \\
\hline 0.8 & 1.3 & 2.261 & 1982.488 & 27-Jun-82 & 0.4 \\
\hline-0.3 & 1.2 & 2.411 & 1982.638 & 21-Aug-82 & -0.1 \\
\hline 2.1 & 0.7 & 2.617 & 1982.844 & 4-Nov-82 & 0.8 \\
\hline 1.0 & 1.9 & 2.814 & 1983.041 & 15-Jan-83 & 0.4 \\
\hline 1.7 & 0.5 & 3.110 & 1983.337 & 3-May-83 & 0.5 \\
\hline-0.5 & 3.0 & 3.294 & 1983.521 & 9-Jul-83 & -0.2 \\
\hline-1.3 & 1.6 & 3.526 & 1983.753 & 2-Oct-83 & -0.4 \\
\hline-1.4 & 0.8 & 3.825 & 1984.052 & 19-Jan-84 & -0.4 \\
\hline-1.6 & 2.2 & 3.983 & 1984.210 & 17-Mar-84 & -0.4 \\
\hline-0.7 & 1.2 & 4.101 & 1984.328 & 29-Apr-84 & -0.2 \\
\hline-0.5 & 1.2 & 4.196 & 1984.423 & 3-Jun-84 & -0.1 \\
\hline-1.3 & 1.6 & 4.421 & 1984.648 & 24-Aug-84 & -0.3 \\
\hline-0.5 & 0.4 & 4.598 & 1984.825 & $28-O c t-84$ & -0.1 \\
\hline-0.8 & 0.6 & 4.713 & 1984.940 & 9-Dec-84 & -0.2 \\
\hline-1.0 & 0.6 & 4.825 & 1985.052 & 19-Jan-85 & -0.2 \\
\hline-0.8 & 0.6 & 5.039 & 1985.266 & 7-Apr-85 & -0.2 \\
\hline 0.6 & 1.0 & 5.231 & 1985.458 & 16-Jun-85 & 0.1 \\
\hline 1.6 & 0.6 & 5.362 & 1985.589 & 3-Aug-85 & 0.3 \\
\hline 0.3 & 0.4 & 5.554 & 1985.781 & $12-$-Oct-85 & 0.1 \\
\hline-1.4 & 0.6 & 5.841 & 1986.068 & 25-Jan-86 & -0.2 \\
\hline 0.2 & 0.3 & 5.959 & 1986.186 & 9-Mar-86 & 0.0 \\
\hline-0.8 & 0.8 & 6.222 & 1986.449 & 13-Jun-86 & -0.1 \\
\hline-0.4 & 1.4 & 6.343 & 1986.570 & 27-Jul-86 & -0.1 \\
\hline-1.3 & 1.0 & 6.587 & 1986.814 & 24-Oct-86 & -0.2 \\
\hline-2.4 & 1.3 & 6.743 & 1986.970 & 20-Dec-86 & -0.4 \\
\hline-0.4 & 0.4 & 6.954 & 1987.181 & 7-Mar-87 & -0.1 \\
\hline-1.7 & 1.0 & 7.294 & 1987.521 & 9-Jul-87 & -0.2 \\
\hline-0.6 & 1.2 & 7.376 & 1987.603 & 8-Aug-87 & -0.1 \\
\hline-1.1 & 0.9 & 7.568 & 1987.795 & 17-Oct-87 & -0.1 \\
\hline-3.2 & 0.5 & 7.858 & 1988.085 & 31-Jan-88 & -0.4 \\
\hline-0.8 & 0.8 & 8.084 & 1988.311 & 23-Apr-88 & -0.1 \\
\hline
\end{tabular}




\begin{tabular}{|c|c|c|c|c|c|}
\hline 0.3 & 0.8 & 8.268 & 1988.495 & 29-Jun-88 & 0.0 \\
\hline-0.3 & 1.2 & 8.467 & 1988.694 & 10-Sep-88 & 0.0 \\
\hline 0.6 & 1.0 & 8.639 & 1988.866 & 12-Nov-88 & 0.1 \\
\hline-1.3 & 1.1 & 8.869 & 1989.096 & 4-Feb-89 & -0.1 \\
\hline-2.2 & 0.8 & 8.935 & 1989.162 & 28-Feb-89 & -0.2 \\
\hline-0.4 & 1.2 & 9.118 & 1989.345 & 6-May-89 & 0.0 \\
\hline-0.7 & 1.7 & 9.313 & 1989.540 & 16-Jul-89 & -0.1 \\
\hline-0.2 & 0.8 & 9.387 & 1989.614 & 12-Aug-89 & 0.0 \\
\hline-1.6 & 2.1 & 9.546 & 1989.773 & 9-Oct-89 & -0.2 \\
\hline-4.7 & 1.9 & 9.573 & 1989.800 & 19-Oct-89 & -0.5 \\
\hline-3.5 & 0.9 & 9.584 & 1989.811 & 23-Oct-89 & -0.4 \\
\hline-2.0 & 1.2 & 9.614 & 1989.841 & 3-Nov-89 & -0.2 \\
\hline-2.0 & 0.9 & 9.647 & 1989.874 & 15-Nov-89 & -0.2 \\
\hline 0.0 & 1.2 & 9.820 & 1990.047 & 17-Jan-90 & 0.0 \\
\hline 0.6 & 0.9 & 9.921 & 1990.148 & 23-Feb-90 & 0.1 \\
\hline-1.5 & 0.8 & 9.998 & 1990.225 & 23-Mar-90 & -0.2 \\
\hline 1.4 & 1.0 & 10.113 & 1990.340 & 4-May-90 & 0.1 \\
\hline-0.2 & 0.6 & 10.255 & 1990.482 & 25-Jun-90 & 0.0 \\
\hline-0.3 & 1.0 & 10.346 & 1990.573 & 28-Jul-90 & 0.0 \\
\hline 0.3 & 1.5 & 10.455 & 1990.682 & 6-Sep-90 & 0.0 \\
\hline 0.0 & 1.0 & 10.570 & 1990.797 & 18-Oct-90 & 0.0 \\
\hline 0.6 & 1.6 & 10.825 & 1991.052 & 19-Jan-91 & 0.1 \\
\hline 0.6 & 1.1 & 11.017 & 1991.244 & 30-Mar-91 & 0.1 \\
\hline 0.9 & 0.9 & 11.170 & 1991.397 & 25-May-91 & 0.1 \\
\hline 1.4 & 1.6 & 11.305 & 1991.532 & 13-Jul-91 & 0.1 \\
\hline 2.4 & 1.9 & 11.573 & 1991.800 & 19-Oct-91 & 0.2 \\
\hline 0.9 & 1.2 & 11.746 & 1991.973 & 21-Dec-91 & 0.1 \\
\hline 1.3 & 1.5 & 11.956 & 1992.183 & 7-Mar-92 & 0.1 \\
\hline 1.4 & 0.7 & 12.150 & 1992.377 & 17-May-92 & 0.1 \\
\hline 0.9 & 1.5 & 12.339 & 1992.566 & 25-Jul-92 & 0.1 \\
\hline 0.3 & 0.9 & 12.511 & 1992.738 & 26-Sep-92 & 0.0 \\
\hline 0.5 & 1.0 & 12.683 & 1992.910 & 28-Nov-92 & 0.0 \\
\hline-3.0 & 1.8 & 12.894 & 1993.121 & 13-Feb-93 & -0.2 \\
\hline-1.1 & 0.9 & 13.200 & 1993.427 & 5-Jun-93 & -0.1 \\
\hline-1.9 & 0.9 & 13.488 & 1993.715 & 18-Sep-93 & -0.1 \\
\hline-2.2 & 0.8 & 13.836 & 1994.063 & 23-Jan-94 & -0.2 \\
\hline-1.2 & 1.2 & 13.968 & 1994.195 & 12-Mar-94 & -0.1 \\
\hline-0.6 & 1.4 & 14.181 & 1994.408 & 29-May-94 & 0.0 \\
\hline-0.8 & 0.9 & 14.370 & 1994.597 & 6-Aug-94 & -0.1 \\
\hline-4.9 & 1.0 & 14.674 & 1994.901 & 25-Nov-94 & -0.3 \\
\hline-5.4 & 1.3 & 14.891 & 1995.118 & 12-Feb-95 & -0.4 \\
\hline-2.3 & 1.0 & 15.173 & 1995.400 & 26-May-95 & -0.2 \\
\hline-2.5 & 1.8 & 15.518 & 1995.745 & 29-Sep-95 & -0.2 \\
\hline-1.9 & 0.9 & 15.792 & 1996.019 & 7-Jan-96 & -0.1 \\
\hline-4.6 & 1.1 & 15.981 & 1996.208 & 16-Mar-96 & -0.3 \\
\hline-4.2 & 0.6 & 16.287 & 1996.514 & 6-Jul-96 & -0.3 \\
\hline-4.2 & 0.5 & 16.497 & 1996.724 & 21-Sep-96 & -0.3 \\
\hline-3.8 & 1.6 & 16.707 & 1996.934 & 7-Dec-96 & -0.2 \\
\hline-3.4 & 0.9 & 16.976 & 1997.203 & 15-Mar-97 & -0.2 \\
\hline
\end{tabular}




$\begin{array}{llllcl}-4.2 & 0.8 & 17.283 & 1997.510 & \text { 5-Jul-97 } & -0.2 \\ -3.4 & 1.1 & 17.551 & 1997.778 & 11-\text { Oct-97 } & -0.2 \\ -4.7 & 0.6 & 17.839 & 1998.066 & \text { 24-Jan-98 } & -0.3 \\ -4.7 & 0.5 & 18.069 & 1998.296 & 18 \text {-Apr-98 } & -0.3 \\ -4.6 & 0.6 & 18.357 & 1998.584 & \text { 1-Aug-98 } & -0.3 \\ -6.5 & 0.9 & 18.740 & 1998.967 & 19-\text { Dec-98 } & -0.3 \\ -3.5 & 0.4 & 18.970 & 1999.197 & 13-\text { Mar-99 } & -0.2 \\ -3.5 & 0.6 & 19.222 & 1999.449 & 13-J u n-99 & -0.2 \\ -4.5 & 1.7 & 19.565 & 1999.792 & 16-\text { Oct-99 } & -0.2 \\ -3.5 & 0.9 & 19.798 & 2000.025 & \text { 9-Jan-00 } & -0.2 \\ -3.4 & 1.1 & 20.142 & 2000.369 & \text { 14-May-00 } & -0.2 \\ -4.8 & 1.2 & 20.254 & 2000.481 & \text { 24-Jun-00 } & -0.2 \\ -1.4 & 1.4 & 20.522 & 2000.749 & \text { 30-Sep-00 } & -0.1 \\ -3.2 & 1.1 & 20.809 & 2001.036 & \text { 13-Jan-01 } & -0.2\end{array}$




\section{SF-11 Worrell Road in Antioch "Antioch" Fault}

\begin{tabular}{|c|c|c|c|c|c|}
\hline $\begin{array}{l}\text { movement } \\
\quad(\mathrm{mm})\end{array}$ & std. dev. & \# of years & year & date & $\begin{array}{c}\mathrm{mm} / \mathrm{yr}-\text { simple } \\
\text { average }\end{array}$ \\
\hline 0.0 & 1.0 & 0.000 & 1980.342 & 4-May-80 & \\
\hline-0.8 & 1.3 & 0.150 & 1980.492 & 28-Jun-80 & \\
\hline-2.5 & 1.7 & 0.379 & 1980.721 & 20-Sep-80 & \\
\hline-2.8 & 1.4 & 0.475 & 1980.817 & $25-O c t-80$ & \\
\hline-2.3 & 1.2 & 0.551 & 1980.893 & 22-Nov-80 & \\
\hline-1.9 & 1.4 & 0.655 & 1980.997 & 30-Dec-80 & \\
\hline-2.9 & 1.0 & 0.762 & 1981.104 & 7-Feb-81 & \\
\hline-2.8 & 0.7 & 1.031 & 1981.373 & 16-May-81 & -2.7 \\
\hline-3.0 & 0.4 & 1.211 & 1981.553 & 21-Jul-81 & -2.5 \\
\hline-3.4 & 0.7 & 1.398 & 1981.740 & 27-Sep-81 & -2.4 \\
\hline-3.4 & 1.0 & 1.707 & 1982.049 & 18-Jan-82 & -2.0 \\
\hline-3.2 & 0.9 & 1.965 & 1982.307 & 22-Apr-82 & -1.6 \\
\hline-3.4 & 0.7 & 2.113 & 1982.455 & 15-Jun-82 & -1.6 \\
\hline-4.9 & 1.6 & 2.321 & 1982.663 & 30-Aug-82 & -2.1 \\
\hline-1.7 & 1.3 & 2.685 & 1983.027 & 10-Jan-83 & -0.6 \\
\hline-3.0 & 0.9 & 2.987 & 1983.329 & 30-Apr-83 & -1.0 \\
\hline-4.0 & 1.4 & 3.096 & 1983.438 & 9-Jun-83 & -1.3 \\
\hline-4.4 & 2.4 & 3.231 & 1983.573 & 28-Jul-83 & -1.4 \\
\hline-7.3 & 1.3 & 3.507 & 1983.849 & 6-Nov-83 & -2.1 \\
\hline-3.7 & 1.3 & 3.721 & 1984.063 & 23-Jan-84 & -1.0 \\
\hline-5.0 & 0.4 & 4.021 & 1984.363 & 12-May-84 & -1.2 \\
\hline-5.0 & 0.8 & 4.117 & 1984.459 & 16-Jun-84 & -1.2 \\
\hline-8.9 & 0.7 & 4.388 & 1984.730 & 23-Sep-84 & -2.0 \\
\hline-6.3 & 0.8 & 4.538 & 1984.880 & 17-Nov-84 & -1.4 \\
\hline-7.2 & 0.5 & 4.770 & 1985.112 & 10-Feb-85 & -1.5 \\
\hline-4.8 & 0.2 & 5.000 & 1985.342 & 5-May-85 & -1.0 \\
\hline-6.0 & 0.3 & 5.154 & 1985.496 & 30-Jun-85 & -1.2 \\
\hline-8.5 & 0.4 & 5.250 & 1985.592 & 4-Aug-85 & -1.6 \\
\hline-7.8 & 0.4 & 5.521 & 1985.863 & 11-Nov-85 & -1.4 \\
\hline-4.2 & 0.5 & 5.746 & 1986.088 & 1-Feb-86 & -0.7 \\
\hline-2.4 & 1.1 & 5.940 & 1986.282 & 13-Apr-86 & -0.4 \\
\hline-3.4 & 1.3 & 6.102 & 1986.444 & 11-Jun-86 & -0.6 \\
\hline-9.0 & 1.2 & 6.458 & 1986.800 & 19-Oct-86 & -1.4 \\
\hline-6.3 & 0.3 & 6.918 & 1987.260 & 5-Apr-87 & -0.9 \\
\hline-7.9 & 1.7 & 7.203 & 1987.545 & 18-Jul-87 & -1.1 \\
\hline-8.5 & 0.9 & 7.474 & 1987.816 & $25-$ Oct-97 & -1.1 \\
\hline-3.5 & 0.3 & 7.759 & 1988.101 & 6-Feb-88 & -0.5 \\
\hline-6.1 & 1.0 & 8.087 & 1988.429 & 5-Jun-88 & -0.8 \\
\hline-8.0 & 1.4 & 8.371 & 1988.713 & 17-Sep-88 & -1.0 \\
\hline-6.6 & 0.5 & 8.620 & 1988.962 & 17-Dec-88 & -0.8 \\
\hline-3.8 & 1.3 & 8.872 & 1989.214 & 19-Mar-89 & -0.4 \\
\hline-6.8 & 2.3 & 9.179 & 1989.521 & 9-Jul-89 & -0.7 \\
\hline-6.7 & 1.7 & 9.425 & 1989.767 & 7-Oct-89 & -0.7 \\
\hline-5.2 & 1.6 & 9.636 & 1989.978 & 23-Dec-89 & -0.5 \\
\hline-6.3 & 0.6 & 9.811 & 1990.153 & 25-Feb-90 & -0.6 \\
\hline-6.3 & 0.8 & 10.003 & 1990.345 & 6-May-90 & -0.6 \\
\hline
\end{tabular}




\begin{tabular}{|c|c|c|c|c|c|}
\hline-7.3 & 0.2 & 10.157 & 1990.499 & 1-Jul-90 & -0.7 \\
\hline-5.9 & 0.4 & 10.406 & 1990.748 & 30-Sep-90 & -0.6 \\
\hline-6.9 & 1.5 & 10.537 & 1990.879 & 17-Nov-90 & -0.7 \\
\hline-6.7 & 0.5 & 10.790 & 1991.132 & 17-Feb-91 & -0.6 \\
\hline-5.0 & 0.3 & 11.036 & 1991.378 & 18-May-91 & -0.5 \\
\hline-7.2 & 0.8 & 11.228 & 1991.570 & 27-Jul-91 & -0.6 \\
\hline-6.6 & 0.9 & 11.420 & 1991.762 & 5-Oct-91 & -0.6 \\
\hline-5.3 & 0.6 & 11.611 & 1991.953 & 14-Dec-91 & -0.5 \\
\hline-1.0 & 0.8 & 11.825 & 1992.167 & 1-Mar-92 & -0.1 \\
\hline-2.8 & 1.4 & 12.013 & 1992.355 & 9-May-92 & -0.2 \\
\hline-4.4 & 1.5 & 12.204 & 1992.546 & 18-Jul-92 & -0.4 \\
\hline-6.1 & 1.2 & 12.360 & 1992.702 & 13-Sep-92 & -0.5 \\
\hline-5.6 & 1.8 & 12.491 & 1992.833 & 31-Oct-92 & -0.4 \\
\hline-0.7 & 2.0 & 12.740 & 1993.082 & 30-Jan-93 & -0.1 \\
\hline-0.8 & 1.7 & 12.970 & 1993.312 & 24-Apr-93 & -0.1 \\
\hline-6.5 & 0.8 & 13.318 & 1993.660 & 29-Aug-93 & -0.5 \\
\hline-7.4 & 1.1 & 13.833 & 1994.175 & 5-Mar-94 & -0.5 \\
\hline-7.0 & 0.5 & 14.044 & 1994.386 & 21-May-94 & -0.5 \\
\hline-6.5 & 3.2 & 14.313 & 1994.655 & 27-Aug-94 & -0.5 \\
\hline-5.6 & 1.0 & 14.562 & 1994.904 & 26-Nov-94 & -0.4 \\
\hline-2.8 & 0.8 & 14.754 & 1995.096 & 4-Feb-95 & -0.2 \\
\hline-3.3 & 2.0 & 15.102 & 1995.444 & 11-Jun-95 & -0.2 \\
\hline-4.4 & 2.1 & 15.444 & 1995.786 & 14-Oct-95 & -0.3 \\
\hline-4.7 & 3.3 & 15.674 & 1996.016 & 6-Jan-96 & -0.3 \\
\hline-5.3 & 1.5 & 16.076 & 1996.418 & 1-Jun-96 & -0.3 \\
\hline-6.2 & 1.1 & 16.344 & 1996.686 & 7-Sep-96 & -0.4 \\
\hline-3.5 & 1.1 & 16.592 & 1996.934 & 7-Dec-96 & -0.2 \\
\hline-4.5 & 1.4 & 16.861 & 1997.203 & 15-Mar-97 & -0.3 \\
\hline-4.5 & 0.8 & 17.168 & 1997.510 & 5-Jul-97 & -0.3 \\
\hline-6.2 & 1.5 & 17.436 & 1997.778 & 11-Oct-97 & -0.4 \\
\hline-3.6 & 0.6 & 17.724 & 1998.066 & 24-Jan-98 & -0.2 \\
\hline-3.7 & 1.4 & 17.954 & 1998.296 & 18-Apr-98 & -0.2 \\
\hline-3.2 & 0.9 & 18.242 & 1998.584 & 1-Aug-98 & -0.2 \\
\hline-4.6 & 2.3 & 18.668 & 1998.970 & 20-Dec-98 & -0.2 \\
\hline-2.8 & 0.9 & 18.970 & 1999.312 & 24-Apr-99 & -0.1 \\
\hline-6.4 & 1.9 & 19.239 & 1999.581 & 31-Jul-99 & -0.3 \\
\hline-3.6 & 2.0 & 19.526 & 1999.868 & 13-Nov-99 & -0.2 \\
\hline-5.3 & 1.5 & 19.816 & 2000.158 & 27-Feb-00 & -0.3 \\
\hline
\end{tabular}


SF-12 D Street in Hayward Hayward Fault 44.56 km

\begin{tabular}{|c|c|c|c|c|c|}
\hline $\begin{array}{l}\text { movement } \\
(\mathrm{mm})\end{array}$ & std. dev. & \# of years & year & date & $\begin{array}{c}\mathrm{mm} / \mathrm{yr} \text {-simple } \\
\text { average }\end{array}$ \\
\hline 0.0 & 0.9 & 0.000 & 1980.478 & 23-Jun-80 & \\
\hline 0.5 & 1.0 & 0.115 & 1980.593 & 4-Aug-80 & \\
\hline 0.2 & 1.8 & 0.235 & 1980.713 & 17-Sep-80 & \\
\hline-0.3 & 0.8 & 0.383 & 1980.861 & 10-Nov-80 & \\
\hline 0.0 & 1.0 & 0.538 & 1981.016 & 6-Jan-81 & \\
\hline 1.8 & 0.6 & 0.667 & 1981.145 & 22-Feb-81 & \\
\hline 1.8 & 0.9 & 0.736 & 1981.214 & 19-Mar-81 & \\
\hline 4.6 & 0.8 & 1.023 & 1981.501 & 2-Jul-81 & 4.5 \\
\hline 4.0 & 0.8 & 1.108 & 1981.586 & 2-Aug-81 & 3.6 \\
\hline 5.3 & 1.3 & 1.358 & 1981.836 & 1-Nov-81 & 3.9 \\
\hline 6.5 & 0.6 & 1.541 & 1982.019 & 7-Jan-82 & 4.2 \\
\hline 6.3 & 0.5 & 1.697 & 1982.175 & 5-Mar-82 & 3.7 \\
\hline 7.4 & 0.3 & 1.938 & 1982.416 & 1-Jun-82 & 3.8 \\
\hline 8.7 & 1.2 & 2.100 & 1982.578 & 30-Jul-82 & 4.1 \\
\hline 9.1 & 1.0 & 2.180 & 1982.658 & 28-Aug-82 & 4.2 \\
\hline 9.1 & 0.3 & 2.355 & 1982.833 & 31-Oct-82 & 3.9 \\
\hline 9.7 & 0.5 & 2.544 & 1983.022 & 8-Jan-83 & 3.8 \\
\hline 11.0 & 0.4 & 2.793 & 1983.271 & 9-Apr-83 & 3.9 \\
\hline 13.3 & 1.7 & 2.938 & 1983.416 & 1-Jun-83 & 4.5 \\
\hline 11.9 & 0.4 & 2.966 & 1983.444 & 11-Jun-83 & 4.0 \\
\hline 13.7 & 1.9 & 3.114 & 1983.592 & 4-Aug-83 & 4.4 \\
\hline 16.4 & 1.7 & 3.237 & 1983.715 & 18-Sep-83 & 5.1 \\
\hline 14.3 & 0.7 & 3.333 & 1983.811 & 23-Oct-83 & 4.3 \\
\hline 12.8 & 0.9 & 3.618 & 1984.096 & 4-Feb-84 & 3.5 \\
\hline 16.3 & 0.8 & 3.773 & 1984.251 & 1-Apr-84 & 4.3 \\
\hline 16.5 & 0.4 & 3.959 & 1984.437 & 8-Jun-84 & 4.2 \\
\hline 18.5 & 1.1 & 4.172 & 1984.650 & 25-Aug-84 & 4.4 \\
\hline 20.3 & 0.7 & 4.328 & 1984.806 & 21-Oct-84 & 4.7 \\
\hline 19.8 & 0.7 & 4.544 & 1985.022 & 8-Jan-85 & 4.4 \\
\hline 20.9 & 0.4 & 4.673 & 1985.151 & 24-Feb-85 & 4.5 \\
\hline 22.6 & 0.7 & 4.845 & 1985.323 & 28-Apr-85 & 4.7 \\
\hline 25.2 & 0.4 & 4.960 & 1985.438 & 9-Jun-85 & 5.1 \\
\hline 25.5 & 0.6 & 5.226 & 1985.704 & 14-Sep-85 & 4.9 \\
\hline 25.6 & 1.4 & 5.495 & 1985.973 & 21-Dec-85 & 4.7 \\
\hline 27.2 & 1.2 & 5.744 & 1986.222 & 22-Mar-86 & 4.7 \\
\hline 27.9 & 0.8 & 5.821 & 1986.299 & 19-Apr-86 & 4.8 \\
\hline 25.6 & 0.7 & 5.947 & 1986.425 & 4-Jun-86 & 4.3 \\
\hline 28.7 & 1.2 & 6.089 & 1986.567 & 26-Jul-86 & 4.7 \\
\hline 29.6 & 1.4 & 6.245 & 1986.723 & 21-Sep-86 & 4.7 \\
\hline 29.7 & 0.8 & 6.380 & 1986.858 & 9-Nov-86 & 4.7 \\
\hline 30.1 & 0.8 & 6.549 & 1987.027 & 10-Jan-87 & 4.6 \\
\hline 31.3 & 0.8 & 6.763 & 1987.241 & 29-Mar-87 & 4.6 \\
\hline 32.0 & 1.8 & 6.952 & 1987.430 & 6-Jun-87 & 4.6 \\
\hline 33.2 & 0.4 & 7.127 & 1987.605 & 9-Aug-87 & 4.7 \\
\hline 33.5 & 1.6 & 7.319 & 1987.797 & 18-Oct-87 & 4.6 \\
\hline 33.3 & 1.2 & 7.585 & 1988.063 & 23-Jan-88 & 4.4 \\
\hline
\end{tabular}




\begin{tabular}{|c|c|c|c|c|c|}
\hline 34.8 & 0.6 & 7.738 & 1988.216 & 19-Mar-88 & 4.5 \\
\hline 38.1 & 1.3 & 7.894 & 1988.372 & 15-May-88 & 4.8 \\
\hline 38.5 & 0.6 & 8.085 & 1988.563 & 24-Jun-88 & 4.8 \\
\hline 40.6 & 1.4 & 8.197 & 1988.675 & 3-Sep-88 & 5.0 \\
\hline 38.5 & 0.7 & 8.372 & 1988.850 & 6-Nov-88 & 4.6 \\
\hline 38.8 & 0.7 & 8.580 & 1989.058 & 21-Jan-89 & 4.5 \\
\hline 42.0 & 0.9 & 8.848 & 1989.326 & 29-Apr-89 & 4.7 \\
\hline 44.0 & 1.5 & 9.095 & 1989.573 & 28-Jul-89 & 4.8 \\
\hline 45.1 & 0.6 & 9.270 & 1989.748 & 30-Sep-89 & 4.9 \\
\hline 44.5 & 0.8 & 9.369 & 1989.847 & 5-Nov-89 & 4.7 \\
\hline 43.8 & 0.5 & 9.541 & 1990.019 & 7-Jan-90 & 4.6 \\
\hline 45.0 & 1.1 & 9.659 & 1990.137 & 19-Feb-90 & 4.7 \\
\hline 45.3 & 1.1 & 9.804 & 1990.282 & 13-Apr-90 & 4.6 \\
\hline 45.6 & 1.1 & 9.919 & 1990.397 & 25-May-90 & 4.6 \\
\hline 46.6 & 0.9 & 10.034 & 1990.512 & 6-Jul-90 & 4.6 \\
\hline 45.7 & 1.7 & 10.133 & 1990.611 & 11-Aug-90 & 4.5 \\
\hline 45.5 & 1.3 & 10.281 & 1990.759 & 4-Oct-90 & 4.4 \\
\hline 44.1 & 0.8 & 10.440 & 1990.918 & 1-Dec-90 & 4.2 \\
\hline 46.7 & 0.7 & 10.651 & 1991.129 & 16-Feb-91 & 4.4 \\
\hline 46.8 & 1.1 & 10.760 & 1991.238 & 28-Mar-91 & 4.3 \\
\hline 47.3 & 0.4 & 10.862 & 1991.340 & 4-May-91 & 4.4 \\
\hline 47.8 & 0.9 & 11.056 & 1991.534 & 14-Jul-91 & 4.3 \\
\hline 49.5 & 1.1 & 11.245 & 1991.723 & 21-Sep-91 & 4.4 \\
\hline 49.5 & 1.0 & 11.418 & 1991.896 & 23-Nov-91 & 4.3 \\
\hline 50.4 & 0.6 & 11.590 & 1992.068 & 25-Jan-92 & 4.3 \\
\hline 54.4 & 0.4 & 11.782 & 1992.260 & 4-Apr-92 & 4.6 \\
\hline 53.1 & 0.9 & 11.858 & 1992.336 & 2-May-92 & 4.5 \\
\hline 54.7 & 1.1 & 12.049 & 1992.527 & 11-Jul-92 & 4.5 \\
\hline 54.4 & 1.8 & 12.221 & 1992.699 & 12-Sep-92 & 4.5 \\
\hline 52.8 & 0.6 & 12.394 & 1992.872 & 14-Nov-92 & 4.3 \\
\hline 53.3 & 1.0 & 12.588 & 1993.066 & 24-Jan-93 & 4.2 \\
\hline 56.5 & 1.7 & 12.738 & 1993.216 & 20-Mar-93 & 4.4 \\
\hline 59.1 & 2.2 & 13.103 & 1993.581 & 31-Jul-93 & 4.5 \\
\hline 57.4 & 1.2 & 13.390 & 1993.868 & 13-Nov-93 & 4.3 \\
\hline 58.7 & 0.8 & 13.582 & 1994.060 & 22-Jan-94 & 4.3 \\
\hline 61.1 & 1.1 & 13.774 & 1994.252 & 2-Apr-94 & 4.4 \\
\hline 60.5 & 1.0 & 13.985 & 1994.463 & 18-Jun-94 & 4.3 \\
\hline 61.7 & 0.7 & 14.111 & 1994.589 & 3-Aug-94 & 4.4 \\
\hline 63.0 & 0.7 & 14.330 & 1994.808 & $22-O c t-94$ & 4.4 \\
\hline 64.2 & 1.7 & 14.599 & 1995.077 & 28-Jan-95 & 4.4 \\
\hline 67.0 & 0.5 & 14.790 & 1995.268 & 8-Apr-95 & 4.5 \\
\hline 66.9 & 0.8 & 14.982 & 1995.460 & 17-Jun-95 & 4.5 \\
\hline 66.9 & 0.5 & 15.166 & 1995.644 & 23-Aug-95 & 4.4 \\
\hline 67.0 & 0.8 & 15.347 & 1995.825 & $28-O c t-95$ & 4.4 \\
\hline 68.1 & 1.7 & 15.538 & 1996.016 & 6-Jan-96 & 4.4 \\
\hline 69.0 & 2.2 & 15.730 & 1996.208 & 16-Mar-96 & 4.4 \\
\hline 70.8 & 1.5 & 15.940 & 1996.418 & 1-Jun-96 & 4.4 \\
\hline 70.6 & 1.7 & 16.161 & 1996.639 & 21-Aug-96 & 4.4 \\
\hline 71.5 & 0.8 & 16.342 & 1996.820 & $26-0 c t-96$ & 4.4 \\
\hline
\end{tabular}




\begin{tabular}{|c|c|c|c|c|}
\hline 69.8 & 0.9 & 16.495 & 1996.973 & 21-Dec-96 \\
\hline 73.3 & 0.4 & 16.651 & 1997.129 & 16-Feb-97 \\
\hline 73.9 & 0.8 & 16.919 & 1997.397 & 25-May-97 \\
\hline 76.2 & 1.2 & 17.054 & 1997.532 & 13-Jul-97 \\
\hline 84.7 & 0.3 & 18.969 & 1999.447 & 12-Jun-99 \\
\hline 85.0 & 0.8 & 19.180 & 1999.658 & 28-Aug-99 \\
\hline 82.9 & 0.8 & 19.352 & 1999.830 & 30-Oct-99 \\
\hline 82.8 & 1.4 & 19.563 & 2000.041 & 15-Jan-00 \\
\hline 83.5 & 1.0 & 19.735 & 2000.213 & 18-Mar-00 \\
\hline 88.0 & 1.5 & 19.926 & 2000.404 & 27-May-00 \\
\hline 85.2 & 2.2 & 20.101 & 2000.579 & 30-Jul-00 \\
\hline 8.1 & 0.5 & 20.347 & 2000.825 & 28-Oct-00 \\
\hline 1.4 & 0.8 & 20.538 & 2001.016 & 6-Jan-01 \\
\hline
\end{tabular}




\section{SF-13 Rose Street in Hayward Hayward Fault 43.22 km}

\begin{tabular}{|c|c|c|c|c|c|}
\hline $\begin{array}{l}\text { movement } \\
(\mathrm{mm})\end{array}$ & std. dev. & \# of years & year & date & $\begin{array}{c}\mathrm{mm} / \mathrm{yr} \text {-simple } \\
\text { average }\end{array}$ \\
\hline 0.0 & 1.2 & 0.000 & 1980.481 & 24-Jun-80 & \\
\hline-1.5 & 1.1 & 0.112 & 1980.593 & 4-Aug-80 & \\
\hline-0.9 & 1.2 & 0.284 & 1980.765 & $6-0 c t-80$ & \\
\hline-2.3 & 1.6 & 0.380 & 1980.861 & 10-Nov-80 & \\
\hline 0.2 & 1.1 & 0.535 & 1981.016 & 6-Jan-81 & \\
\hline 2.7 & 0.4 & 0.733 & 1981.214 & 19-Mar-81 & \\
\hline 3.5 & 0.5 & 1.020 & 1981.501 & 2-Jul-81 & 3.4 \\
\hline 2.6 & 0.8 & 1.105 & 1981.586 & 2-Aug-81 & 2.4 \\
\hline 5.4 & 0.8 & 1.355 & 1981.836 & 1-Nov-81 & 4.0 \\
\hline 12.6 & 0.6 & 1.538 & 1982.019 & 7-Jan-82 & 8.2 \\
\hline 9.6 & 0.2 & 1.694 & 1982.175 & 5-Mar-82 & 5.7 \\
\hline 11.9 & 1.6 & 1.738 & 1982.219 & 21-Mar-82 & 6.8 \\
\hline 10.3 & 0.9 & 1.935 & 1982.416 & 1-Jun-82 & 5.3 \\
\hline 9.4 & 0.6 & 2.097 & 1982.578 & 30-Jul-82 & 4.5 \\
\hline 9.2 & 0.5 & 2.177 & 1982.658 & 28-Aug-82 & 4.2 \\
\hline 7.2 & 0.6 & 2.352 & 1982.833 & 31-Oct-82 & 3.1 \\
\hline 9.9 & 1.2 & 2.541 & 1983.022 & 8-Jan-83 & 3.9 \\
\hline 13.5 & 0.6 & 2.790 & 1983.271 & 9-Apr-83 & 4.8 \\
\hline 14.6 & 1.1 & 2.933 & 1983.414 & 31-May-83 & 5.0 \\
\hline 12.2 & 0.6 & 2.963 & 1983.444 & 11-Jun-83 & 4.1 \\
\hline 14.2 & 3.1 & 3.111 & 1983.592 & 4-Aug-83 & 4.6 \\
\hline 13.4 & 1.3 & 3.234 & 1983.715 & 18-Sep-83 & 4.1 \\
\hline 13.4 & 1.7 & 3.330 & 1983.811 & 23-Oct-83 & 4.0 \\
\hline 15.4 & 0.4 & 3.585 & 1984.066 & 24-Jan-84 & 4.3 \\
\hline 19.3 & 0.9 & 3.768 & 1984.249 & 31-Mar-84 & 5.1 \\
\hline 19.9 & 0.5 & 3.956 & 1984.437 & 8-Jun-84 & 5.0 \\
\hline 19.2 & 1.0 & 4.169 & 1984.650 & 25-Aug-84 & 4.6 \\
\hline 18.0 & 1.0 & 4.325 & 1984.806 & $21-$ Oct-84 & 4.2 \\
\hline 20.8 & 0.8 & 4.541 & 1985.022 & 8-Jan-85 & 4.6 \\
\hline 22.3 & 0.6 & 4.670 & 1985.151 & $24-F e b-85$ & 4.8 \\
\hline 23.5 & 0.4 & 4.842 & 1985.323 & 28-Apr-85 & 4.9 \\
\hline 24.4 & 1.6 & 4.957 & 1985.438 & 9-Jun-85 & 4.9 \\
\hline 22.0 & 1.2 & 5.223 & 1985.704 & 14-Sep-85 & 4.2 \\
\hline 23.9 & 1.3 & 5.492 & 1985.973 & 21-Dec-85 & 4.4 \\
\hline 34.9 & 0.8 & 5.741 & 1986.222 & 22-Mar-86 & 6.1 \\
\hline 34.3 & 1.2 & 5.818 & 1986.299 & 19-Apr-86 & 5.9 \\
\hline 33.0 & 0.1 & 5.944 & 1986.425 & 4-Jun-86 & 5.6 \\
\hline 30.8 & 0.3 & 6.086 & 1986.567 & 26-Jul-86 & 5.1 \\
\hline 30.2 & 1.4 & 6.242 & 1986.723 & $21-$ Sep-86 & 4.8 \\
\hline 31.0 & 0.8 & 6.377 & 1986.858 & 9-Nov-86 & 4.9 \\
\hline 30.0 & 1.3 & 6.546 & 1987.027 & 10-Jan-87 & 4.6 \\
\hline 35.5 & 1.6 & 6.760 & 1987.241 & 29-Mar-87 & 5.3 \\
\hline 36.5 & 0.9 & 6.949 & 1987.430 & 6-Jun-87 & 5.3 \\
\hline 34.7 & 1.4 & 7.124 & 1987.605 & 9-Aug-87 & 4.9 \\
\hline 34.9 & 1.5 & 7.316 & 1987.797 & 18-Oct-87 & 4.8 \\
\hline 34.8 & 1.3 & 7.582 & 1988.063 & 23-Jan-88 & 4.6 \\
\hline
\end{tabular}




\begin{tabular}{|c|c|c|c|c|c|}
\hline 34.5 & 1.2 & 7.735 & 1988.216 & 19-Mar-88 & 4.5 \\
\hline 32.9 & 1.3 & 7.891 & 1988.372 & 15-May-88 & 4.2 \\
\hline 35.8 & 1.7 & 8.082 & 1988.563 & 24-Jul-88 & 4.4 \\
\hline 37.9 & 1.7 & 8.194 & 1988.675 & 3-Sep-88 & 4.6 \\
\hline 38.4 & 1.3 & 8.369 & 1988.850 & 6-Nov-88 & 4.6 \\
\hline 39.5 & 1.1 & 8.577 & 1989.058 & 21-Jan-89 & 4.6 \\
\hline 44.9 & 1.4 & 8.845 & 1989.326 & 29-Apr-89 & 5.1 \\
\hline 42.7 & 1.4 & 9.092 & 1989.573 & 28-Jul-89 & 4.7 \\
\hline 42.8 & 0.9 & 9.267 & 1989.748 & 30-Sep-89 & 4.6 \\
\hline 44.9 & 0.8 & 9.366 & 1989.847 & 5-Nov-89 & 4.8 \\
\hline 43.6 & 0.4 & 9.538 & 1990.019 & 7-Jan-90 & 4.6 \\
\hline 46.3 & 0.8 & 9.656 & 1990.137 & 19-Feb-90 & 4.8 \\
\hline 47.1 & 0.5 & 9.801 & 1990.282 & 13-Apr-90 & 4.8 \\
\hline 44.0 & 1.1 & 9.916 & 1990.397 & 25-May-90 & 4.4 \\
\hline 43.5 & 0.3 & 10.031 & 1990.512 & 6-Jul-90 & 4.3 \\
\hline 43.5 & 0.6 & 10.130 & 1990.611 & 11-Aug-90 & 4.3 \\
\hline 44.9 & 1.6 & 10.278 & 1990.759 & $4-O c t-90$ & 4.4 \\
\hline 46.3 & 0.8 & 10.437 & 1990.918 & 1-Dec-90 & 4.4 \\
\hline 46.8 & 0.5 & 10.648 & 1991.129 & 16-Feb-91 & 4.4 \\
\hline 48.8 & 0.7 & 10.757 & 1991.238 & 28-Mar-91 & 4.5 \\
\hline 50.3 & 0.8 & 10.859 & 1991.340 & 4-May-91 & 4.6 \\
\hline 54.6 & 0.6 & 11.053 & 1991.534 & 14-Jul-91 & 4.9 \\
\hline 54.7 & 1.0 & 11.242 & 1991.723 & 21-Sep-91 & 4.9 \\
\hline 57.0 & 0.6 & 11.415 & 1991.896 & 23-Nov-91 & 5.0 \\
\hline 60.0 & 0.5 & 11.587 & 1992.068 & 25-Jan-92 & 5.2 \\
\hline 61.5 & 0.8 & 11.779 & 1992.260 & 4-Apr-92 & 5.2 \\
\hline 61.4 & 0.4 & 11.855 & 1992.336 & 2-Мay-92 & 5.2 \\
\hline 59.3 & 0.7 & 12.046 & 1992.527 & 11-Jul-92 & 4.9 \\
\hline 59.3 & 1.9 & 12.251 & 1992.732 & 24-Sep-92 & 4.8 \\
\hline 59.6 & 1.7 & 12.391 & 1992.872 & 14-Nov-92 & 4.8 \\
\hline 62.1 & 1.2 & 12.585 & 1993.066 & 24-Jan-93 & 4.9 \\
\hline 65.0 & 2.1 & 12.735 & 1993.216 & 20-Mar-93 & 5.1 \\
\hline 65.9 & 1.8 & 12.949 & 1993.430 & 6-Jun-93 & 5.1 \\
\hline 67.6 & 0.4 & 13.198 & 1993.679 & 5-Sep-93 & 5.1 \\
\hline 62.1 & 2.7 & 13.371 & 1993.852 & 7-Nov-93 & 4.6 \\
\hline 65.1 & 1.0 & 13.563 & 1994.044 & 16-Jan-94 & 4.8 \\
\hline 65.6 & 1.5 & 13.755 & 1994.236 & 27-Mar-94 & 4.8 \\
\hline 72.3 & 0.9 & 13.982 & 1994.463 & 18-Jun-94 & 5.2 \\
\hline 67.1 & 0.7 & 14.108 & 1994.589 & 3-Aug-94 & 4.8 \\
\hline 67.3 & 0.9 & 14.327 & 1994.808 & 22-Oct-94 & 4.7 \\
\hline 76.0 & 0.9 & 14.596 & 1995.077 & 28-Jan-95 & 5.2 \\
\hline 74.1 & 1.6 & 14.787 & 1995.268 & 8-Apr-95 & 5.0 \\
\hline 73.4 & 1.1 & 14.979 & 1995.460 & 17-Jun-95 & 4.9 \\
\hline 72.8 & 0.7 & 15.163 & 1995.644 & 23-Aug-95 & 4.8 \\
\hline 75.7 & 2.2 & 15.344 & 1995.825 & 28-Oct-95 & 4.9 \\
\hline 76.0 & 2.1 & 15.497 & 1995.978 & 23-Dec-95 & 4.9 \\
\hline 75.0 & 0.9 & 15.634 & 1996.115 & 11-Feb-96 & 4.8 \\
\hline 75.8 & 0.7 & 15.784 & 1996.265 & 6-Apr-96 & 4.8 \\
\hline 78.8 & 1.5 & 16.014 & 1996.495 & 29-Jun-96 & 4.9 \\
\hline
\end{tabular}




\begin{tabular}{|c|c|c|c|c|c|}
\hline 80.5 & 1.1 & 16.153 & 1996.634 & 19-Aug-96 & 5.0 \\
\hline 78.1 & 0.7 & 16.339 & 1996.820 & 26-Oct-96 & 4.8 \\
\hline 82.8 & 1.0 & 16.549 & 1997.030 & 11-Jan-97 & 5.0 \\
\hline 79.0 & 2.2 & 16.760 & 1997.241 & 29-Mar-97 & 4.7 \\
\hline 83.7 & 0.7 & 16.916 & 1997.397 & 25-May-97 & 4.9 \\
\hline 82.9 & 0.9 & 17.051 & 1997.532 & 13-Jul-97 & 4.9 \\
\hline 86.1 & 0.9 & 17.152 & 1997.633 & 19-Aug-97 & 5.0 \\
\hline 82.9 & 0.9 & 17.355 & 1997.836 & 1-Nov-97 & 4.8 \\
\hline 88.5 & 0.8 & 17.546 & 1998.027 & 10-Jan-98 & 5.0 \\
\hline 85.7 & 0.8 & 17.719 & 1998.200 & 14-Mar-98 & 4.8 \\
\hline 87.9 & 0.6 & 17.933 & 1998.414 & 31-May-98 & 4.9 \\
\hline 88.6 & 0.8 & 18.218 & 1998.699 & 12-Sep-98 & 4.9 \\
\hline 91.3 & 0.4 & 18.374 & 1998.855 & 8-Nov-98 & 5.0 \\
\hline 92.3 & 0.7 & 18.582 & 1999.063 & 23-Jan-99 & 5.0 \\
\hline 96.0 & 1.3 & 18.719 & 1999.200 & 14-Mar-99 & 5.1 \\
\hline 94.1 & 1.2 & 18.892 & 1999.373 & 16-May-99 & 5.0 \\
\hline 92.9 & 1.8 & 19.177 & 1999.658 & 28-Aug-99 & 4.8 \\
\hline 92.3 & 0.6 & 19.349 & 1999.830 & 30-Oct-99 & 4.8 \\
\hline 98.9 & 0.4 & 19.560 & 2000.041 & 15-Jan-00 & 5.1 \\
\hline 98.2 & 0.5 & 19.770 & 2000.251 & 1-Apr-00 & 5.0 \\
\hline 99.0 & 0.8 & 19.981 & 2000.462 & 17-Jun-00 & 5.0 \\
\hline 99.1 & 1.1 & 20.136 & 2000.617 & 13-Aug-00 & 4.9 \\
\hline 103.1 & 1.1 & 20.344 & 2000.825 & $28-0 c t-00$ & 5.1 \\
\hline 98.3 & 1.2 & 20.535 & 2001.016 & 6-Jan-01 & 4.8 \\
\hline
\end{tabular}


SF-14 Point Reyes at PR National Seashore Headquarters

movement

( $\mathrm{mm})$

0.0

0.1

0.3

0.6

0.3

1.3

$-0.1$

1.2

1.1

$-0.4$

0.6

0.9

1.3

0.5

1.5

1.0

1.1

1.3

1.3

1.4

1.9

2.5

1.3

3.2

2.0

2.6

2.7

2.7

4.3

3.5

2.5

3.5

3.7

3.5

3.6

3.1

3.7

5.4

5.2

5.6

5.6

3.6

2.6

4.5

4.5

3.3 std. dev.

1.1

0.8

0.5

0.1

0.0

0.3

0.5

0.4

0.6

0.3

0.6

0.6

0.8

1.5

0.8

0.9

0.4

1.1

0.7

0.5

1.4

0.8

0.7

0.6

0.8

0.7

0.8

0.7

0.8

0.7

1.6

1.0

1.0

1.0

0.8

0.2

0.7

1.5

0.5

0.6

1.2

0.2

0.3

0.7

0.3

0.9
\# of years

0.000

0.211

0.364

0.479

0.731

1.005

1.255

1.353

1.474

1.701

2.085

2.372

2.507

2.699

2.989

3.215

3.464

3.598

3.770

4.000

4.057

4.249

4.444

4.518

4.655

4.726

4.931

5.115

5.225

5.405

5.534

5.649

5.786

6.014

6.205

6.378

6.531

6.723

6.953

7.221

7.412

7.661

7.967

8.389

8.600

8.986
San Andreas Fault

$\begin{array}{ccc}\text { year } & \text { date } & \begin{array}{c}\mathrm{mm} / \mathrm{yr} \text {-simple } \\ \text { average }\end{array} \\ 1985.096 & \text { 4-Feb-85 } & \\ 1985.307 & \text { 22-Apr-85 } & \\ 1985.460 & \text { 17-Jun-85 } & \end{array}$

1985.460 17-Jun-85

1985.575 29-Jul-85

$1985.827 \quad 29-$ Oct-85

$1986.101 \quad 6-F e b-86$

1986.351

1986.449

1986.570

1986.797

1987.181

1987.468

1987.603

1987.795

1988.085

1988.311

1988.560

1988.694

1988.866

1989.096

1989.153

1989.345

1989.540

1989.614

1989.751

1989.822

1990.027

1990.211

1990.321

1990.501

1990.630

1990.745

1990.882

1991.110

1991.301

1991.474

1991.627

1991.819

1992.049

1992.317

1992.508

1992.757

1993.063

1993.485

1993.696

1994.082

\section{8-May-86}

13-Jun-86

27-Jul-86

18-Oct-86

7-Mar-87

20-Jun-87

8-Aug-87

17-Oct-87

31-Jan-88

23-Apr-88

23-Jul-88

10-Sep-88

12-Nov-88

4-Feb-89

25-Feb-89

6-May-89

16-Jul-89

12-Aug-89

1-Oct-89

27-Oct-89

10-Jan-90

18-Mar-90

27-Apr-90

2-Jul-90

18-Aug-90

29-Sep-90

18-Nov-90

9-Feb-91

20-Apr-91

22-Jun-91

17-Aug-91

26-Oct-91

18-Jan-92

25-Apr-92

4-Jul-92

3-Oct-92

23-Jan-93

26-Jun-93

11-Sep-93

30-Jan-94

1.3

$-0.1$

0.9

0.7

$-0.2$

0.3

0.4

0.5

0.2

0.5

0.3

0.3

0.4

0.3

0.3

0.5

0.6

0.3

0.7

0.4

0.5

0.5

0.5

0.8

0.6

0.5

0.6

0.6

0.6

0.6

0.5

0.6

0.8

0.7

0.8

0.8

0.5

0.3

0.5

0.5

0.4 


\begin{tabular}{|c|c|c|c|c|}
\hline 5.7 & 0.4 & 9.194 & 1994.290 & 16-Apr-94 \\
\hline 6.3 & 0.5 & 9.427 & 1994.523 & 10-Jul-94 \\
\hline 5.9 & 0.3 & 9.805 & 1994.901 & 25-Nov-94 \\
\hline 4.7 & 0.3 & 10.022 & 1995.118 & 12-Feb-95 \\
\hline 4.3 & 1.0 & 10.304 & 1995.400 & 26-May-95 \\
\hline 4.0 & 0.7 & 10.594 & 1995.690 & 9-Sep-95 \\
\hline 3.8 & 0.4 & 10.805 & 1995.901 & 25-Nov-95 \\
\hline 3.9 & 1.1 & 10.997 & 1996.093 & 3-Feb-96 \\
\hline 4.0 & 1.0 & 11.284 & 1996.380 & 18-Мау-96 \\
\hline 3.0 & 3.0 & 11.418 & 1996.514 & 6-Jul-96 \\
\hline 3.0 & 1.0 & 11.628 & 1996.724 & 21-Sep-96 \\
\hline 4.7 & 0.8 & 11.838 & 1996.934 & 7-Dec-96 \\
\hline 6.5 & 0.8 & 12.068 & 1997.164 & 1-Mar-97 \\
\hline 3.9 & 0.4 & 12.320 & 1997.416 & 1-Jun-97 \\
\hline 3.3 & 1.0 & 12.625 & 1997.721 & 20-Sep-97 \\
\hline 3.6 & 0.3 & 12.896 & 1997.992 & 28-Dec-97 \\
\hline 3.2 & 1.5 & 13.296 & 1998.392 & 23-May-98 \\
\hline 2.3 & 1.3 & 13.545 & 1998.641 & 22-Aug-98 \\
\hline 3.4 & 0.9 & 13.833 & 1998.929 & 5-Dec-98 \\
\hline 3.0 & 0.8 & 14.066 & 1999.162 & 28-Feb-99 \\
\hline 1.6 & 0.5 & 14.236 & 1999.332 & 1-May-99 \\
\hline 2.6 & 0.4 & 14.542 & 1999.638 & 21-Aug-99 \\
\hline 3.3 & 0.4 & 14.852 & 1999.948 & 12-Dec-99 \\
\hline 3.3 & 1.2 & 15.155 & 2000.251 & 1-Apr-00 \\
\hline 2.4 & 1.2 & 15.385 & 2000.481 & 24-Jun-00 \\
\hline 1.9 & 1.8 & 15.653 & 2000.749 & 30-Sep-00 \\
\hline 0.9 & 2.0 & 15.940 & 2001.036 & 13-Jan-01 \\
\hline
\end{tabular}




\section{SF-15 Linda Vista Ave in Napa West Napa Fault}

\begin{tabular}{|c|c|c|c|c|c|}
\hline $\begin{array}{l}\text { movement } \\
\quad(\mathrm{mm})\end{array}$ & std. dev. & \# of years & year & date & $\begin{array}{c}\mathrm{mm} / \mathrm{yr}-\text { simple } \\
\text { average }\end{array}$ \\
\hline 0.0 & 2.4 & 0.000 & 1980.568 & 26-Jul-80 & \\
\hline 2.1 & 1.3 & 0.268 & 1980.836 & 1-Nov-80 & \\
\hline-3.9 & 1.2 & 0.446 & 1981.014 & 5-Jan-81 & \\
\hline-5.1 & 0.8 & 0.670 & 1981.238 & 28-Mar-81 & \\
\hline 2.3 & 0.6 & 0.966 & 1981.534 & 14-Jul-81 & 2.4 \\
\hline 4.3 & 0.9 & 1.054 & 1981.622 & 15-Aug-81 & 4.1 \\
\hline 4.3 & 0.4 & 1.290 & 1981.858 & 9-Nov-81 & 3.3 \\
\hline-2.0 & 0.4 & 1.503 & 1982.071 & 26-Jan-82 & -1.3 \\
\hline-1.8 & 1.5 & 1.769 & 1982.337 & 3-May-82 & -1.0 \\
\hline 3.7 & 0.8 & 1.944 & 1982.512 & 6-Jul-82 & 1.9 \\
\hline 8.4 & 0.8 & 2.092 & 1982.660 & 29-Aug-82 & 4.0 \\
\hline-1.4 & 2.1 & 2.320 & 1982.888 & 20-Nov-82 & -0.6 \\
\hline-2.2 & 2.2 & 2.500 & 1983.068 & 25-Jan-83 & -0.9 \\
\hline-2.3 & 1.2 & 2.764 & 1983.332 & 1-May-83 & -0.8 \\
\hline 0.2 & 3.4 & 2.940 & 1983.532 & 13-Jul-83 & 0.1 \\
\hline-3.0 & 1.1 & 3.481 & 1984.049 & 18-Jan-84 & -0.9 \\
\hline-5.0 & 0.7 & 3.798 & 1984.366 & 13-May-84 & -1.3 \\
\hline-1.8 & 1.2 & 3.987 & 1984.555 & 21-Jul-84 & -0.5 \\
\hline-1.8 & 1.6 & 4.372 & 1984.940 & 9-Dec-84 & -0.4 \\
\hline-1.5 & 1.5 & 4.564 & 1985.132 & 17-Feb-85 & -0.3 \\
\hline-4.1 & 1.9 & 4.794 & 1985.362 & 12-May-85 & -0.9 \\
\hline 4.5 & 0.9 & 4.969 & 1985.537 & 15-Jul-85 & 0.9 \\
\hline 2.9 & 0.5 & 5.254 & 1985.822 & $27-O c t-85$ & 0.6 \\
\hline 0.7 & 0.5 & 5.487 & 1986.055 & 20-Jan-86 & 0.1 \\
\hline-0.2 & 1.0 & 5.673 & 1986.241 & 29-Mar-86 & 0.0 \\
\hline 0.9 & 0.5 & 6.172 & 1986.740 & 27-Sep-86 & 0.1 \\
\hline-3.8 & 1.4 & 6.344 & 1986.912 & 29-Nov-86 & -0.6 \\
\hline-3.4 & 1.2 & 6.577 & 1987.145 & 22-Feb-87 & -0.5 \\
\hline-3.1 & 1.1 & 6.747 & 1987.315 & $25-A p r-87$ & -0.5 \\
\hline-1.1 & 1.2 & 6.920 & 1987.488 & 27-Jun-87 & -0.2 \\
\hline 0.2 & 1.3 & 7.081 & 1987.649 & 25-Aug-87 & 0.0 \\
\hline-3.0 & 2.0 & 7.459 & 1988.027 & 10-Jan-88 & -0.4 \\
\hline-2.1 & 0.7 & 7.727 & 1988.295 & 17-Apr-88 & -0.3 \\
\hline-0.3 & 1.9 & 7.880 & 1988.448 & 12-Jun-88 & 0.0 \\
\hline 5.5 & 1.2 & 8.090 & 1988.658 & 28-Aug-88 & 0.7 \\
\hline 9.4 & 0.6 & 8.243 & 1988.811 & 23-Oct-88 & 1.1 \\
\hline-0.5 & 1.4 & 8.424 & 1988.992 & 28-Dec-88 & -0.1 \\
\hline 1.5 & 1.4 & 8.627 & 1989.195 & 12-Mar-89 & 0.2 \\
\hline-1.8 & 0.7 & 8.854 & 1989.422 & 3-Jun-89 & -0.2 \\
\hline 3.3 & 1.5 & 8.988 & 1989.556 & 22-Jul-89 & 0.4 \\
\hline 5.7 & 0.5 & 9.218 & 1989.786 & 14-Oct-89 & 0.6 \\
\hline 0.1 & 0.8 & 9.353 & 1989.921 & 2-Dec-89 & 0.0 \\
\hline-1.0 & 1.4 & 9.473 & 1990.041 & 15-Jan-90 & -0.1 \\
\hline-1.3 & 0.7 & 9.643 & 1990.211 & 18-Mar-90 & -0.1 \\
\hline 0.0 & 0.9 & 9.755 & 1990.323 & 28-Apr-90 & 0.0 \\
\hline-0.1 & 0.6 & 9.890 & 1990.458 & 16-Jun-90 & 0.0 \\
\hline
\end{tabular}




\begin{tabular}{|c|c|c|c|c|c|}
\hline 5.0 & 1.0 & 10.024 & 1990.592 & 4-Aug-90 & 0.5 \\
\hline 2.8 & 1.0 & 10.407 & 1990.975 & 22-Dec-90 & 0.3 \\
\hline 3.6 & 0.6 & 10.640 & 1991.208 & 17-Mar-91 & 0.3 \\
\hline 3.6 & 0.9 & 10.810 & 1991.378 & 18-May-91 & 0.3 \\
\hline 2.9 & 4.1 & 11.002 & 1991.570 & 27-Jul-91 & 0.3 \\
\hline 8.5 & 3.9 & 11.194 & 1991.762 & 5-Oct-91 & 0.8 \\
\hline 7.7 & 1.3 & 11.385 & 1991.953 & 14-Dec-91 & 0.7 \\
\hline 6.0 & 1.8 & 11.599 & 1992.167 & 1-Mar-92 & 0.5 \\
\hline 4.3 & 0.4 & 11.787 & 1992.355 & 9-May-92 & 0.4 \\
\hline 18.4 & 2.0 & 11.978 & 1992.546 & 18-Jul-92 & 1.5 \\
\hline 27.3 & 2.6 & 12.093 & 1992.661 & 29-Aug-92 & 2.3 \\
\hline 20.4 & 1.3 & 12.284 & 1992.852 & 7-Nov-92 & 1.7 \\
\hline 12.2 & 3.3 & 12.536 & 1993.104 & 7-Feb-93 & 1.0 \\
\hline 6.0 & 0.5 & 12.955 & 1993.523 & 10-Jul-93 & 0.5 \\
\hline 6.0 & 1.2 & 13.166 & 1993.734 & 25-Sep-93 & 0.5 \\
\hline-2.6 & 0.5 & 13.607 & 1994.175 & 5-Mar-94 & -0.2 \\
\hline-3.1 & 0.6 & 13.818 & 1994.386 & 21-May-94 & -0.2 \\
\hline 4.3 & 1.3 & 14.087 & 1994.655 & 27-Aug-94 & 0.3 \\
\hline 2.9 & 1.1 & 14.224 & 1994.792 & 16-Oct-94 & 0.2 \\
\hline-0.1 & 1.8 & 14.405 & 1994.973 & 21-Dec-94 & 0.0 \\
\hline-1.4 & 2.7 & 14.605 & 1995.173 & 4-Mar-95 & -0.1 \\
\hline-2.4 & 0.9 & 14.876 & 1995.444 & 11-Jun-95 & -0.2 \\
\hline-3.9 & 0.7 & 15.295 & 1995.863 & 11-Nov-95 & -0.3 \\
\hline-4.2 & 1.4 & 15.525 & 1996.093 & 3-Feb-96 & -0.3 \\
\hline-7.5 & 1.6 & 15.812 & 1996.380 & 18-Мay-96 & -0.5 \\
\hline-6.4 & 0.7 & 16.022 & 1996.590 & 3-Aug-96 & -0.4 \\
\hline-5.9 & 1.1 & 16.290 & 1996.858 & 9-Nov-96 & -0.4 \\
\hline-7.2 & 1.4 & 16.596 & 1997.164 & 1-Mar-97 & -0.4 \\
\hline-3.0 & 1.0 & 16.848 & 1997.416 & 1-Jun-97 & -0.2 \\
\hline 13.5 & 1.3 & 17.153 & 1997.721 & 20-Sep-97 & 0.8 \\
\hline 5.9 & 0.7 & 17.443 & 1998.011 & 4-Jan-98 & 0.3 \\
\hline-1.1 & 1.1 & 17.766 & 1998.334 & 2-May-98 & -0.1 \\
\hline 2.6 & 0.7 & 18.207 & 1998.775 & 10-Oct-98 & 0.1 \\
\hline 0.5 & 1.2 & 18.476 & 1999.044 & 16-Jan-99 & 0.0 \\
\hline
\end{tabular}


SF-16 Nielson Road in Santa Rosa Rodgers Creek Fault

$\begin{array}{cccccc}\begin{array}{c}\text { movement } \\ (\mathrm{mm})\end{array} & \text { std. dev. } & \begin{array}{c}\text { \# of years } \\ \text { d }\end{array} & \begin{array}{c}\text { year } \\ \text { date }\end{array} & \begin{array}{c}\text { mm } \text { average } \\ \text { averample }\end{array} \\ 2.0 & 2.7 & 0.000 & 1980.628 & \text { 17-Aug-80 } & \\ -5.1 & 0.9 & 0.208 & 1980.836 & \text { 1-Nov-80 } & \\ -1.9 & 2.6 & 0.386 & 1981.014 & \text { 5-Jan-81 } & \\ 4.1 & 1.5 & 0.610 & 1981.238 & \text { 28-Mar-81 } & \\ 4.2 & 1.6 & 0.906 & 1981.534 & \text { 14-Jul-81 } & \\ 1.9 & 1.1 & 0.994 & 1981.622 & \text { 15-Aug-81 } & \\ 0.8 & 0.8 & 1.191 & 1981.819 & \text { 26-Oct-81 } & 1.6 \\ 0.1 & 2.1 & 1.443 & 1982.071 & \text { 26-Jan-82 } & 0.6 \\ 4.4 & 1.8 & 1.709 & 1982.337 & \text { 3-May-82 } & 0.1 \\ 0.6 & 0.5 & 1.884 & 1982.512 & \text { 6-Jul-82 } & 2.3 \\ 3.5 & 1.0 & 2.032 & 1982.660 & \text { 29-Aug-82 } & 0.3 \\ 0.9 & 3.7 & 2.260 & 1982.888 & \text { 20-Nov-82 } & 1.5 \\ 4.0 & 2.1 & 2.440 & 1983.068 & \text { 25-Jan-83 } & 0.4 \\ 5.3 & 2.7 & 2.704 & 1983.332 & \text { 1-May-83 } & 1.5 \\ 3.3 & 3.7 & 2.904 & 1983.532 & \text { 13-Jul-83 } & 1.8 \\ 5.1 & 3.1 & 3.145 & 1983.773 & \text { 9-Oct-83 } & 1.0 \\ 6.6 & 1.3 & 3.738 & 1984.366 & \text { 13-May-84 } & 1.4 \\ -4.5 & 0.8 & 3.927 & 1984.555 & \text { 21-Jul-84 } & 1.7 \\ 0.6 & 1.3 & 4.216 & 1984.844 & \text { 4-Nov-84 } & -1.1 \\ 7.0 & 1.4 & 4.504 & 1985.132 & \text { 17-Feb-85 } & 0.1 \\ 7.4 & 0.9 & 4.734 & 1985.362 & \text { 12-May-85 } & 1.5 \\ -5.1 & 5.2 & 4.909 & 1985.537 & \text { 15-Jul-85 } & 1.5 \\ & 1.5 & 5.427 & 1986.055 & \text { 20-Jan-86 } & -0.9\end{array}$




\section{SF-17 Contra Costa College in San Pablo Hayward Fault 4.49 km}

\begin{tabular}{|c|c|c|c|c|c|}
\hline $\begin{array}{l}\text { movement } \\
(\mathrm{mm})\end{array}$ & std. dev. & \# of years & year & date & $\begin{array}{c}\mathrm{mm} / \mathrm{yr} \text {-simple } \\
\text { average }\end{array}$ \\
\hline 1.0 & 0.8 & 0.000 & 1980.609 & 10-Aug-80 & \\
\hline 2.0 & 1.1 & 0.287 & 1980.896 & 23-Nov-80 & \\
\hline 1.2 & 1.8 & 0.440 & 1981.049 & 18-Jan-81 & \\
\hline 3.4 & 0.7 & 0.975 & 1981.584 & 1-Aug-81 & 3.5 \\
\hline-5.6 & 0.5 & 1.323 & 1981.932 & 6-Dec-81 & -4.2 \\
\hline-7.8 & 0.4 & 1.438 & 1982.047 & 17-Jan-82 & -5.4 \\
\hline-6.8 & 0.4 & 1.706 & 1982.315 & 25-Apr-82 & -4.0 \\
\hline-1.0 & 0.5 & 1.851 & 1982.460 & 17-Jun-82 & -0.5 \\
\hline 1.9 & 0.1 & 1.975 & 1982.584 & 1-Aug-82 & 1.0 \\
\hline-0.1 & 0.4 & 2.416 & 1983.025 & 9-Jan-83 & 0.0 \\
\hline 4.1 & 0.7 & 2.780 & 1983.389 & 22-May-83 & 1.5 \\
\hline 11.7 & 1.2 & 2.933 & 1983.542 & 17-Jul-83 & 4.0 \\
\hline 12.0 & 0.5 & 2.991 & 1983.600 & 7-Aug-83 & 4.0 \\
\hline 9.4 & 0.6 & 3.336 & 1983.945 & $11-$ Dec-83 & 2.8 \\
\hline 6.2 & 0.8 & 3.451 & 1984.060 & 22-Jan-84 & 1.8 \\
\hline 15.5 & 1.1 & 3.642 & 1984.251 & 1-Apr-84 & 4.3 \\
\hline 23.0 & 0.7 & 3.948 & 1984.557 & 22-Jul-84 & 5.8 \\
\hline 20.3 & 0.4 & 4.216 & 1984.825 & $28-O c t-84$ & 4.8 \\
\hline 15.1 & 0.1 & 4.465 & 1985.074 & 27-Jan-85 & 3.4 \\
\hline 20.9 & 0.2 & 4.657 & 1985.266 & 7-Apr-85 & 4.5 \\
\hline 24.6 & 0.1 & 4.829 & 1985.438 & 9-Jun-85 & 5.1 \\
\hline 22.2 & 0.2 & 5.021 & 1985.630 & 18-Aug-85 & 4.4 \\
\hline 22.5 & 0.5 & 5.232 & 1985.841 & 3-Nov-85 & 4.3 \\
\hline 16.5 & 0.7 & 5.481 & 1986.090 & 2-Feb-86 & 3.0 \\
\hline 18.8 & 0.7 & 5.654 & 1986.263 & 6-Apr-86 & 3.3 \\
\hline 26.8 & 0.7 & 5.846 & 1986.455 & 15-Jun-86 & 4.6 \\
\hline 28.1 & 0.9 & 6.057 & 1986.666 & 31-Aug-86 & 4.6 \\
\hline 27.1 & 0.1 & 6.172 & 1986.781 & $12-0 c t-86$ & 4.4 \\
\hline 24.5 & 0.7 & 6.459 & 1987.068 & 25-Jan-87 & 3.8 \\
\hline 25.9 & 0.9 & 6.555 & 1987.164 & 1-Mar-87 & 3.9 \\
\hline 29.6 & 0.8 & 6.862 & 1987.471 & 21-Jun-87 & 4.3 \\
\hline 29.7 & 0.6 & 7.054 & 1987.663 & 30-Aug-87 & 4.2 \\
\hline 27.1 & 0.8 & 7.301 & 1987.910 & 28-Nov-87 & 3.7 \\
\hline 25.1 & 0.5 & 7.495 & 1988.104 & 7-Feb-88 & 3.4 \\
\hline 29.4 & 0.4 & 7.705 & 1988.314 & 24-Apr-88 & 3.8 \\
\hline 32.6 & 0.6 & 7.916 & 1988.525 & 10-Jul-88 & 4.1 \\
\hline 34.1 & 1.1 & 8.107 & 1988.716 & 18-Sep-88 & 4.2 \\
\hline 33.2 & 1.0 & 8.317 & 1988.926 & 4-Dec-88 & 4.0 \\
\hline 32.5 & 1.0 & 8.470 & 1989.079 & 29-Jan-89 & 3.8 \\
\hline 33.4 & 0.9 & 8.662 & 1989.271 & 9-Apr-89 & 3.9 \\
\hline 36.6 & 0.5 & 8.854 & 1989.463 & 18-Jun-89 & 4.1 \\
\hline 38.0 & 0.8 & 8.988 & 1989.597 & 6-Aug-89 & 4.2 \\
\hline 40.5 & 0.5 & 9.257 & 1989.866 & 12-Nov-89 & 4.4 \\
\hline 38.8 & 0.5 & 9.449 & 1990.058 & 21-Jan-90 & 4.1 \\
\hline 37.7 & 0.3 & 9.564 & 1990.173 & 4-Mar-90 & 3.9 \\
\hline 38.8 & 0.6 & 9.698 & 1990.307 & 22-Apr-90 & 4.1 \\
\hline
\end{tabular}




\begin{tabular}{|c|c|c|c|c|c|}
\hline 39.4 & 0.6 & 9.832 & 1990.441 & 10-Jun-90 & 4.0 \\
\hline 40.9 & 0.8 & 9.966 & 1990.575 & 29-Jul-90 & 4.1 \\
\hline 42.3 & 0.4 & 10.101 & 1990.710 & $16-$ Sep-90 & 4.2 \\
\hline 42.6 & 0.9 & 10.216 & 1990.825 & $28-O c t-90$ & 4.2 \\
\hline 43.5 & 0.3 & 10.465 & 1991.074 & 27-Jan-91 & 4.2 \\
\hline 38.7 & 0.7 & 10.638 & 1991.247 & 31-Mar-91 & 3.6 \\
\hline 39.1 & 1.2 & 10.753 & 1991.362 & 12-May-91 & 3.6 \\
\hline 41.1 & 0.7 & 10.999 & 1991.608 & 10-Aug-91 & 3.7 \\
\hline 41.5 & 1.8 & 11.175 & 1991.784 & 13-Oct-91 & 3.7 \\
\hline 41.6 & 1.3 & 11.328 & 1991.937 & 8-Dec-91 & 3.7 \\
\hline 42.5 & 0.9 & 11.462 & 1992.071 & 26-Jan-92 & 3.7 \\
\hline 43.0 & 1.0 & 11.634 & 1992.243 & 29-Mar-92 & 3.7 \\
\hline 47.7 & 1.0 & 11.825 & 1992.434 & 7-Jun-92 & 4.0 \\
\hline 46.8 & 0.9 & 12.093 & 1992.702 & 13-Sep-92 & 3.9 \\
\hline 45.9 & 0.9 & 12.246 & 1992.855 & 8-Nov-92 & 3.7 \\
\hline 44.9 & 0.8 & 12.418 & 1993.027 & 10-Jan-93 & 3.6 \\
\hline 45.6 & 0.9 & 12.591 & 1993.200 & 14-Mar-93 & 3.6 \\
\hline 49.4 & 0.8 & 12.821 & 1993.430 & 6-Jun-93 & 3.9 \\
\hline 53.8 & 1.1 & 13.070 & 1993.679 & 5-Sep-93 & 4.1 \\
\hline 54.4 & 1.0 & 13.243 & 1993.852 & 7-Nov-93 & 4.1 \\
\hline 55.7 & 1.0 & 13.435 & 1994.044 & 16-Jan-94 & 4.2 \\
\hline 55.0 & 1.4 & 13.627 & 1994.236 & 27-Mar-94 & 4.0 \\
\hline 62.1 & 0.5 & 13.857 & 1994.466 & 19-Jun-94 & 4.5 \\
\hline 61.4 & 0.6 & 13.969 & 1994.578 & 30-Jul-94 & 4.4 \\
\hline 64.4 & 0.4 & 14.183 & 1994.792 & 16-Oct-94 & 4.5 \\
\hline 58.5 & 1.2 & 14.364 & 1994.973 & 21-Dec-94 & 4.1 \\
\hline 59.3 & 0.9 & 14.566 & 1995.175 & 5-Mar-95 & 4.1 \\
\hline 61.6 & 1.1 & 14.854 & 1995.463 & 18-Jun-95 & 4.1 \\
\hline 69.1 & 0.1 & 15.027 & 1995.636 & 20-Aug-95 & 4.6 \\
\hline 70.3 & 2.4 & 15.257 & 1995.866 & 12-Nov-95 & 4.6 \\
\hline 71.3 & 0.5 & 15.331 & 1995.940 & 9-Dec-95 & 4.7 \\
\hline 64.2 & 1.6 & 15.506 & 1996.115 & $11-F e b-96$ & 4.1 \\
\hline 65.8 & 1.2 & 15.697 & 1996.306 & 21-Apr-96 & 4.2 \\
\hline 71.0 & 1.6 & 15.869 & 1996.478 & 23-Jun-96 & 4.5 \\
\hline 74.2 & 0.7 & 16.022 & 1996.631 & 18-Aug-96 & 4.6 \\
\hline 76.9 & 0.5 & 16.213 & 1996.822 & 27-Oct-96 & 4.7 \\
\hline 74.0 & 0.7 & 16.347 & 1996.956 & 15-Dec-96 & 4.5 \\
\hline 73.8 & 0.6 & 16.520 & 1997.129 & 16-Feb-97 & 4.5 \\
\hline 81.5 & 0.9 & 16.712 & 1997.321 & 27-Apr-97 & 4.9 \\
\hline 85.6 & 1.5 & 16.846 & 1997.455 & 15-Jun-97 & 5.1 \\
\hline 85.3 & 0.9 & 17.018 & 1997.627 & 17-Aug-97 & 5.0 \\
\hline 85.2 & 2.0 & 17.210 & 1997.819 & $26-O c t-97$ & 5.0 \\
\hline 78.9 & 0.4 & 17.364 & 1997.973 & 21-Dec-97 & 4.5 \\
\hline 77.7 & 0.5 & 17.517 & 1998.126 & 15-Feb-98 & 4.4 \\
\hline 77.9 & 0.6 & 17.651 & 1998.260 & 5-Apr-98 & 4.4 \\
\hline 78.2 & 0.7 & 17.843 & 1998.452 & 14-Jun-98 & 4.4 \\
\hline 83.2 & 1.4 & 18.054 & 1998.663 & 30-Aug-98 & 4.6 \\
\hline 86.8 & 1.3 & 18.207 & 1998.816 & $25-O c t-98$ & 4.8 \\
\hline 80.8 & 0.4 & 18.323 & 1998.932 & 6-Dec-98 & 4.4 \\
\hline
\end{tabular}




$\begin{array}{llllll}82.0 & 0.8 & 18.495 & 1999.104 & \text { 7-Feb-99 } & 4.4 \\ 81.8 & 1.9 & 18.649 & 1999.258 & \text { 4-Apr-99 } & 4.4 \\ 85.8 & 1.4 & 18.821 & 1999.430 & \text { 6-Jun-99 } & 4.6 \\ 92.3 & 1.7 & 19.051 & 1999.660 & \text { 29-Aug-99 } & 4.8 \\ 92.6 & 1.7 & 19.281 & 1999.890 & 21 \text {-Nov-99 } & 4.8 \\ 92.7 & 0.5 & 19.435 & 2000.044 & 16-\text { Jan-00 } & 4.8 \\ 89.7 & 1.5 & 19.626 & 2000.235 & 26 \text {-Mar-00 } & 4.6 \\ 92.8 & 0.7 & 19.875 & 2000.484 & 25-\text { Jun-00 } & 4.7 \\ 94.9 & 1.7 & 20.008 & 2000.617 & 13-\text { Aug-00 } & 4.7 \\ 94.7 & 0.6 & 20.219 & 2000.828 & 29-\text { Oct-00 } & 4.7 \\ 94.0 & 0.6 & 20.449 & 2001.058 & 21 \text {-Jan-01 } & 4.6\end{array}$


SF-18 Alder Creek near Point Arena San Andreas Fault

$\begin{array}{cccccc}\begin{array}{c}\text { movement } \\ (\mathrm{mm})\end{array} & \text { std. dev. } & \begin{array}{c}\text { \# of years } \\ \text { o.0 }\end{array} & \begin{array}{c}\text { year } \\ \text { date }\end{array} & \begin{array}{c}\text { mm/yr-simple } \\ \text { average }\end{array} \\ 1.3 & 3.3 & 19.000 & 1981.025 & \text { 9-Jan-81 } & \\ 2.6 & 4.1 & 2.112 & 1982.038 & \text { 14-Jan-82 } & 1.3 \\ 3.5 & 4.2 & 3.013 & 1984.137 & \text { 19-Feb-83 } & 1.2 \\ 4.3 & 1.5 & 4.005 & 1985.030 & \text { 14-Jan-84 } & 1.2 \\ 6.4 & 0.9 & 5.022 & 1986.047 & \text { 17-Jan-85 } & 1.1 \\ 5.2 & 0.9 & 6.022 & 1987.047 & \text { 17-Jan-87 } & 1.3 \\ 6.7 & 0.9 & 7.019 & 1988.044 & \text { 16-Jan-88 } & 0.9 \\ 7.4 & 1.9 & 8.013 & 1989.038 & \text { 14-Jan-89 } & 0.9 \\ 7.8 & 1.5 & 9.057 & 1990.082 & \text { 30-Jan-90 } & 0.9 \\ 10.8 & 1.3 & 10.008 & 1991.033 & \text { 12-Jan-91 } & 1.1 \\ 12.2 & 1.7 & 11.060 & 1992.085 & \text { 31-Jan-92 } & 1.1 \\ 11.3 & 3.2 & 12.005 & 1993.030 & \text { 11-Jan-93 } & 0.9 \\ 6.4 & 2.0 & 12.970 & 1993.995 & \text { 29-Dec-93 } & 0.5 \\ 8.4 & 2.4 & 13.967 & 1994.992 & \text { 28-Dec-94 } & 0.6 \\ 9.6 & 2.6 & 15.434 & 1996.459 & \text { 16-Jun-96 } & 0.6 \\ 9.5 & 1.6 & 16.561 & 1997.586 & \text { 2-Aug-97 } & 0.6 \\ 10.1 & 1.4 & 17.578 & 1998.603 & \text { 8-Aug-98 } & 0.6 \\ 11.7 & 1.7 & 18.575 & 1999.600 & \text { 7-Aug-99 } & 0.6 \\ 11.5 & 1.2 & 19.571 & 2000.596 & \text { 5-Aug-00 } & 0.6\end{array}$




\begin{tabular}{|c|c|c|c|c|c|}
\hline $\begin{array}{l}\text { movement } \\
\quad(\mathrm{mm})\end{array}$ & std. dev. & \# of years & year & date & $\begin{array}{cc}\mathrm{mm} / \mathrm{yr} \text {-simple } & \text { average after } \\
\text { average } & 1992.852\end{array}$ \\
\hline 0.0 & 1.2 & 0.000 & 1980.896 & 23-Nov-80 & \\
\hline-0.6 & 0.8 & 0.137 & 1981.033 & 12-Jan-81 & \\
\hline-0.5 & 0.8 & 0.156 & 1981.052 & 19-Jan-81 & \\
\hline-0.4 & 0.6 & 0.381 & 1981.277 & 11-Apr-81 & \\
\hline 0.6 & 0.3 & 0.657 & 1981.553 & 21-Jul-81 & \\
\hline 0.5 & 0.6 & 0.729 & 1981.625 & 16-Aug-81 & 0.7 \\
\hline 0.3 & 0.8 & 1.156 & 1982.052 & 19-Jan-82 & 0.3 \\
\hline 0.8 & 0.7 & 1.419 & 1982.315 & 25-Apr-82 & 0.6 \\
\hline 0.9 & 1.0 & 1.564 & 1982.460 & 17-Jun-82 & 0.6 \\
\hline 2.6 & 1.0 & 1.688 & 1982.584 & 1-Aug-82 & 1.5 \\
\hline 3.0 & 1.1 & 1.742 & 1982.638 & 21-Aug-82 & 1.7 \\
\hline 2.2 & 0.1 & 2.129 & 1983.025 & 9-Jan-83 & 1.0 \\
\hline 2.4 & 0.5 & 2.490 & 1983.386 & 21-May-83 & 1.0 \\
\hline 3.5 & 0.7 & 2.559 & 1983.455 & 15-Jun-83 & 1.4 \\
\hline 4.6 & 1.5 & 2.652 & 1983.548 & 19-Jul-83 & 1.7 \\
\hline 3.3 & 0.8 & 2.704 & 1983.600 & 7-Aug-83 & 1.2 \\
\hline 3.2 & 0.9 & 3.156 & 1984.052 & 19-Jan-84 & 1.0 \\
\hline 3.5 & 0.9 & 3.432 & 1984.328 & $29-A p r-84$ & 1.0 \\
\hline 4.0 & 0.5 & 3.527 & 1984.423 & 3-Jun-84 & 1.1 \\
\hline 1.6 & 0.8 & 3.752 & 1984.648 & 24-Aug-84 & 0.4 \\
\hline 3.8 & 0.9 & 3.888 & 1984.784 & $13-0 c t-84$ & 1.0 \\
\hline 4.4 & 0.5 & 4.238 & 1985.134 & 18-Feb-85 & 1.0 \\
\hline 4.8 & 0.8 & 4.468 & 1985.364 & 13-May-85 & 1.1 \\
\hline 6.3 & 0.6 & 4.603 & 1985.499 & 1-Jul-85 & 1.4 \\
\hline 5.5 & 0.1 & 4.679 & 1985.575 & 29-Jul-85 & 1.2 \\
\hline 4.4 & 0.4 & 4.885 & 1985.781 & 12-Oct-85 & 0.9 \\
\hline 2.2 & 0.2 & 5.172 & 1986.068 & 25-Jan-86 & 0.4 \\
\hline 2.8 & 0.7 & 5.367 & 1986.263 & 6-Apr-86 & 0.5 \\
\hline 2.0 & 0.7 & 5.885 & 1986.781 & $12-0 c t-86$ & 0.3 \\
\hline 2.6 & 1.2 & 6.172 & 1987.068 & 25-Jan-87 & 0.4 \\
\hline 1.4 & 0.6 & 6.364 & 1987.260 & 5-Apr-87 & 0.2 \\
\hline 4.3 & 0.3 & 6.638 & 1987.534 & 14-Jul-87 & 0.6 \\
\hline 1.1 & 1.0 & 7.014 & 1987.910 & 28-Nov-87 & 0.2 \\
\hline 2.7 & 0.8 & 7.208 & 1988.104 & 7-Feb-88 & 0.4 \\
\hline 3.9 & 1.4 & 7.437 & 1988.333 & 1-May-88 & 0.5 \\
\hline 1.5 & 0.4 & 7.601 & 1988.497 & 30-Jun-88 & 0.2 \\
\hline 0.2 & 0.8 & 7.932 & 1988.828 & $29-O c t-88$ & 0.0 \\
\hline 1.1 & 0.9 & 8.181 & 1989.077 & 28-Jan-89 & 0.1 \\
\hline 3.0 & 0.5 & 8.394 & 1989.290 & 16-Apr-89 & 0.4 \\
\hline 2.3 & 0.5 & 8.622 & 1989.518 & 8-Jul-89 & 0.3 \\
\hline 2.0 & 1.2 & 8.970 & 1989.866 & 12-Nov-89 & 0.2 \\
\hline 1.2 & 1.0 & 9.162 & 1990.058 & 21-Jan-90 & 0.1 \\
\hline 2.7 & 0.4 & 9.312 & 1990.208 & 17-Mar-90 & 0.3 \\
\hline 3.0 & 1.0 & 9.370 & 1990.266 & 7-Apr-90 & 0.3 \\
\hline
\end{tabular}




\begin{tabular}{|c|c|c|c|c|c|c|}
\hline 3.3 & 0.9 & 9.463 & 1990.359 & 11-May-90 & 0.3 & \\
\hline 2.3 & 1.3 & 9.660 & 1990.556 & 22-Jul-90 & 0.2 & \\
\hline 3.1 & 1.3 & 9.819 & 1990.715 & 18-Sep-90 & 0.3 & \\
\hline 0.1 & 0.6 & 9.983 & 1990.879 & 17-Nov-90 & 0.0 & \\
\hline 4.8 & 1.0 & 10.236 & 1991.132 & 17-Feb-91 & 0.5 & \\
\hline 4.5 & 0.7 & 10.400 & 1991.296 & 18-Apr-91 & 0.4 & \\
\hline 3.2 & 0.9 & 10.559 & 1991.455 & 15-Jun-91 & 0.3 & \\
\hline 3.2 & 0.6 & 10.657 & 1991.553 & 21-Jul-91 & 0.3 & \\
\hline 3.2 & 0.6 & 10.846 & 1991.742 & 28-Sep-91 & 0.3 & \\
\hline 3.0 & 0.6 & 11.003 & 1991.899 & 24-Nov-91 & 0.3 & \\
\hline 4.3 & 0.8 & 11.156 & 1992.052 & 19-Jan-92 & 0.4 & \\
\hline 5.7 & 1.3 & 11.424 & 1992.320 & 26-Apr-92 & 0.5 & \\
\hline 1.1 & 2.0 & 11.765 & 1992.661 & 29-Aug-92 & 0.1 & \\
\hline 2.7 & 0.5 & 11.956 & 1992.852 & 7-Nov-92 & 0.2 & \\
\hline 6.7 & 1.0 & 12.208 & 1993.104 & 7-Feb-93 & 0.5 & \\
\hline 8.1 & 1.1 & 12.764 & 1993.660 & 29-Aug-93 & 0.6 & \\
\hline 9.4 & 1.2 & 13.030 & 1993.926 & 4-Dec-93 & 0.7 & 6.2 \\
\hline 9.7 & 0.6 & 13.282 & 1994.178 & 6-Mar-94 & 0.7 & 5.3 \\
\hline 12.7 & 0.8 & 13.433 & 1994.329 & 30-Apr-94 & 0.9 & 6.8 \\
\hline 11.5 & 0.6 & 13.699 & 1994.595 & 5-Aug-94 & 0.8 & 5.0 \\
\hline 11.5 & 1.7 & 14.008 & 1994.904 & 26-Nov-94 & 0.8 & 4.3 \\
\hline 17.5 & 0.9 & 14.200 & 1995.096 & 4-Feb-95 & 1.2 & 6.6 \\
\hline 16.6 & 0.7 & 14.488 & 1995.384 & 20-May-95 & 1.1 & 5.5 \\
\hline 17.7 & 0.3 & 14.759 & 1995.655 & 27-Aug-95 & 1.2 & 5.4 \\
\hline 17.5 & 0.9 & 14.970 & 1995.866 & 12-Nov-95 & 1.2 & 4.9 \\
\hline 20.1 & 1.0 & 15.120 & 1996.016 & 6-Jan-96 & 1.3 & 5.5 \\
\hline 19.8 & 0.8 & 15.312 & 1996.208 & 16-Mar-96 & 1.3 & 5.1 \\
\hline 20.1 & 2.9 & 15.522 & 1996.418 & 1-Jun-96 & 1.3 & 4.7 \\
\hline 20.3 & 0.9 & 15.754 & 1996.650 & 25-Aug-96 & 1.3 & 4.6 \\
\hline 21.3 & 0.4 & 16.003 & 1996.899 & 24-Nov-96 & 1.3 & 4.6 \\
\hline 22.0 & 0.6 & 16.134 & 1997.030 & 11-Jan-97 & 1.4 & 4.6 \\
\hline 20.9 & 0.8 & 16.345 & 1997.241 & 29-Mar-97 & 1.3 & 4.1 \\
\hline 22.2 & 0.3 & 16.501 & 1997.397 & 25-Мay-97 & 1.3 & 4.3 \\
\hline 21.6 & 0.9 & 16.652 & 1997.548 & 19-Jul-97 & 1.3 & 4.0 \\
\hline 22.7 & 1.2 & 16.805 & 1997.701 & 13-Sep-97 & 1.4 & 4.1 \\
\hline 24.4 & 0.9 & 16.997 & 1997.893 & 22-Nov-97 & 1.4 & 4.3 \\
\hline 23.9 & 1.0 & 17.249 & 1998.145 & 22-Feb-98 & 1.4 & 4.0 \\
\hline 25.8 & 0.8 & 17.400 & 1998.296 & 18-Apr-98 & 1.5 & 4.2 \\
\hline 23.9 & 1.4 & 17.688 & 1998.584 & 1-Aug-98 & 1.4 & 3.7 \\
\hline 24.6 & 0.6 & 17.860 & 1998.756 & 3-Oct-98 & 1.4 & 3.7 \\
\hline 24.4 & 0.5 & 18.036 & 1998.932 & 6-Dec-98 & 1.4 & 3.6 \\
\hline 27.9 & 0.4 & 18.244 & 1999.140 & 20-Feb-99 & 1.5 & 4.0 \\
\hline 31.0 & 0.2 & 18.416 & 1999.312 & 24-Apr-99 & 1.7 & 4.4 \\
\hline 31.7 & 1.1 & 18.551 & 1999.447 & 12-Jun-99 & 1.7 & 4.4 \\
\hline 31.2 & 1.7 & 18.742 & 1999.638 & 21-Aug-99 & 1.7 & 4.2 \\
\hline 31.8 & 0.5 & 18.899 & 1999.795 & 17-Oct-99 & 1.7 & 4.2 \\
\hline 32.6 & 0.5 & 19.052 & 1999.948 & 12-Dec-99 & 1.7 & 4.2 \\
\hline 31.9 & 1.1 & 19.205 & 2000.101 & 6-Feb-00 & 1.7 & 4.0 \\
\hline 32.5 & 0.6 & 19.413 & 2000.309 & 22-Apr-00 & 1.7 & 4.0 \\
\hline
\end{tabular}




$\begin{array}{llllcll}33.1 & 1.3 & 19.566 & 2000.462 & 17-\text { Jun-00 } & 1.7 & 4.0 \\ 32.9 & 1.1 & 19.721 & 2000.617 & 13 \text {-Aug-00 } & 1.7 & 3.9 \\ 34.3 & 1.0 & 20.044 & 2000.940 & 9 \text {-Dec-00 } & 1.7 & 3.9 \\ 34.9 & 0.4 & 20.216 & 2001.112 & 10 \text {-Feb-01 } & 1.7 & 3.9\end{array}$




\section{SF-20 Red Top Road in Cordelia Green Valley Fault}

\begin{tabular}{|c|c|c|c|c|c|}
\hline $\begin{array}{l}\text { movement } \\
(\mathrm{mm})\end{array}$ & std. dev. & \# of years & year & date & $\begin{array}{c}\mathrm{mm} / \mathrm{yr} \text {-simple } \\
\text { average }\end{array}$ \\
\hline 0.0 & 1.8 & 0.000 & 1984.456 & 15-Jun-84 & \\
\hline 4.7 & 2.2 & 0.347 & 1984.803 & $20-O c t-84$ & \\
\hline 8.1 & 1.1 & 0.618 & 1985.074 & 27-Jan-85 & \\
\hline 3.9 & 2.0 & 0.829 & 1985.285 & 14-Apr-85 & \\
\hline 5.4 & 1.1 & 0.980 & 1985.436 & 8-Jun-85 & 5.5 \\
\hline 9.4 & 2.2 & 1.174 & 1985.630 & 18-Aug-85 & 8.0 \\
\hline 9.4 & 0.3 & 1.385 & 1985.841 & 3-Nov-85 & 6.8 \\
\hline 4.9 & 0.8 & 1.634 & 1986.090 & 2-Feb-86 & 3.0 \\
\hline 19.0 & 2.7 & 2.130 & 1986.586 & 2-Aug-86 & 8.9 \\
\hline 22.5 & 6.7 & 2.210 & 1986.666 & 31-Aug-86 & 10.2 \\
\hline 20.2 & 2.1 & 2.284 & 1986.740 & 27-Sep-86 & 8.8 \\
\hline 19.5 & 3.5 & 2.456 & 1986.912 & 29-Nov-86 & 7.9 \\
\hline 14.0 & 2.0 & 2.708 & 1987.164 & 1-Mar-87 & 5.2 \\
\hline 20.0 & 3.4 & 3.015 & 1987.471 & 21-Jun-87 & 6.6 \\
\hline 17.9 & 1.1 & 3.207 & 1987.663 & 30-Aug-87 & 5.6 \\
\hline 17.3 & 1.5 & 3.571 & 1988.027 & 10-Jan-88 & 4.8 \\
\hline 18.9 & 2.4 & 3.839 & 1988.295 & 17-Apr-88 & 4.9 \\
\hline 22.0 & 2.7 & 3.992 & 1988.448 & 12-Jun-88 & 5.5 \\
\hline 30.9 & 1.5 & 4.372 & 1988.828 & 29-Oct-88 & 7.1 \\
\hline 20.4 & 2.8 & 4.621 & 1989.077 & 28-Jan-89 & 4.4 \\
\hline 20.2 & 1.0 & 4.834 & 1989.290 & 16-Apr-89 & 4.2 \\
\hline 21.7 & 2.0 & 5.141 & 1989.597 & 6-Aug-89 & 4.2 \\
\hline 28.2 & 2.2 & 5.465 & 1989.921 & 2-Dec-89 & 5.2 \\
\hline 34.5 & 1.8 & 5.590 & 1990.041 & 15-Jan-90 & 6.2 \\
\hline 33.2 & 1.2 & 5.733 & 1990.189 & 10-Mar-90 & 5.8 \\
\hline 36.8 & 2.8 & 5.851 & 1990.307 & 22-Apr-90 & 6.3 \\
\hline 37.8 & 2.3 & 5.985 & 1990.441 & 10-Jun-90 & 6.3 \\
\hline 41.0 & 2.8 & 6.174 & 1990.630 & 18-Aug-90 & 6.6 \\
\hline 42.8 & 1.4 & 6.289 & 1990.745 & 29-Sep-90 & 6.8 \\
\hline 42.2 & 2.7 & 6.426 & 1990.882 & 18-Nov-90 & 6.6 \\
\hline 37.3 & 4.7 & 6.654 & 1991.110 & 9-Feb-91 & 5.6 \\
\hline 37.6 & 1.4 & 6.791 & 1991.247 & 31-Mar-91 & 5.5 \\
\hline 42.0 & 3.2 & 6.999 & 1991.455 & 15-Jun-91 & 6.0 \\
\hline 37.6 & 1.5 & 7.152 & 1991.608 & 10-Aug-91 & 5.3 \\
\hline 44.1 & 5.6 & 7.328 & 1991.784 & 13-Oct-91 & 6.0 \\
\hline 37.4 & 1.9 & 7.481 & 1991.937 & 8-Dec-91 & 5.0 \\
\hline 38.9 & 2.2 & 7.615 & 1992.071 & 26-Jan-92 & 5.1 \\
\hline 41.5 & 2.0 & 7.787 & 1992.243 & 29-Mar-92 & 5.3 \\
\hline 42.2 & 2.5 & 7.978 & 1992.434 & 7-Jun-92 & 5.3 \\
\hline 40.9 & 3.6 & 8.148 & 1992.604 & 8-Aug-92 & 5.0 \\
\hline 44.8 & 1.3 & 8.377 & 1992.833 & 31-Oct-92 & 5.3 \\
\hline 43.0 & 2.9 & 8.626 & 1993.082 & 30-Jan-93 & 5.0 \\
\hline 42.8 & 2.9 & 8.856 & 1993.312 & 24-Apr-93 & 4.8 \\
\hline 47.0 & 3.7 & 9.067 & 1993.523 & 10-Jul-93 & 5.2 \\
\hline 53.9 & 1.3 & 9.278 & 1993.734 & $25-$ Sep-93 & 5.8 \\
\hline 51.8 & 2.0 & 9.470 & 1993.926 & 4-Dec-93 & 5.5 \\
\hline
\end{tabular}




\begin{tabular}{|c|c|c|c|c|c|}
\hline 52.3 & 3.4 & 9.722 & 1994.178 & 6-Mar-94 & 5.4 \\
\hline 58.4 & 2.4 & 9.873 & 1994.329 & 30-Apr-94 & 5.9 \\
\hline 56.2 & 2.0 & 10.139 & 1994.595 & 5-Aug-94 & 5.5 \\
\hline 56.5 & 12.3 & 10.295 & 1994.751 & 1-Oct-94 & 5.5 \\
\hline 53.6 & 1.4 & 10.486 & 1994.942 & 10-Dec-94 & 5.1 \\
\hline 49.3 & 2.1 & 10.643 & 1995.099 & 5-Feb-95 & 4.6 \\
\hline 52.4 & 1.0 & 10.717 & 1995.173 & 4-Mar-95 & 4.9 \\
\hline 52.3 & 2.8 & 10.928 & 1995.384 & 20-May-95 & 4.8 \\
\hline 55.5 & 3.8 & 11.199 & 1995.655 & 27-Aug-95 & 5.0 \\
\hline 57.1 & 1.4 & 11.407 & 1995.863 & 11-Nov-95 & 5.0 \\
\hline 52.6 & 1.1 & 11.580 & 1996.036 & 13-Jan-96 & 4.5 \\
\hline 49.8 & 3.5 & 11.793 & 1996.249 & 31-Mar-96 & 4.2 \\
\hline 54.4 & 2.7 & 11.981 & 1996.437 & 8-Jun-96 & 4.5 \\
\hline 58.9 & 1.4 & 12.230 & 1996.686 & 7-Sep-96 & 4.8 \\
\hline 54.7 & 3.4 & 12.443 & 1996.899 & 24-Nov-96 & 4.4 \\
\hline 58.1 & 1.2 & 12.654 & 1997.110 & 9-Feb-97 & 4.6 \\
\hline 58.6 & 4.5 & 12.922 & 1997.378 & 18-May-97 & 4.5 \\
\hline 62.7 & 2.3 & 13.092 & 1997.548 & 19-Jul-97 & 4.8 \\
\hline 62.5 & 0.7 & 13.245 & 1997.701 & 13-Sep-97 & 4.7 \\
\hline 60.4 & 1.9 & 13.536 & 1997.992 & 28-Dec-97 & 4.5 \\
\hline 63.3 & 0.8 & 13.747 & 1998.203 & 15-Mar-98 & 4.6 \\
\hline 67.9 & 2.8 & 13.977 & 1998.433 & 7-Jun-98 & 4.9 \\
\hline 66.1 & 2.9 & 14.130 & 1998.586 & 2-Aug-98 & 4.7 \\
\hline 63.0 & 3.8 & 14.300 & 1998.756 & 3-Oct-98 & 4.4 \\
\hline 64.1 & 1.3 & 14.476 & 1998.932 & 6-Dec-98 & 4.4 \\
\hline 74.6 & 2.8 & 14.706 & 1999.162 & 28-Feb-99 & 5.1 \\
\hline 79.8 & 3.9 & 15.741 & 2000.197 & 12-Mar-00 & 5.1 \\
\hline 84.9 & 1.0 & 15.967 & 2000.423 & 3-Jun-00 & 5.3 \\
\hline 70.5 & 2.9 & 16.200 & 2000.656 & 27-Aug-00 & 4.4 \\
\hline 66.9 & 2.5 & 16.334 & 2000.790 & $15-O c t-00$ & 4.1 \\
\hline 67.5 & 1.4 & 16.487 & 2000.943 & 10-Dec-00 & 4.1 \\
\hline 69.6 & 2.1 & 16.659 & 2001.115 & 11-Feb-01 & 4.2 \\
\hline
\end{tabular}




\section{SF-21 Roberts Road near Penngrove Rodgers Creek Fault}

\begin{tabular}{|c|c|c|c|c|c|}
\hline $\begin{array}{l}\text { movement } \\
(\mathrm{mm})\end{array}$ & std. dev. & \# of years & year & date & $\begin{array}{c}\mathrm{mm} / \mathrm{yr} \text {-simple } \\
\text { average }\end{array}$ \\
\hline 0.0 & 4.1 & 0.000 & 1986.721 & 20-Sep-86 & \\
\hline-4.7 & 2.3 & 0.424 & 1987.145 & 22-Feb-87 & \\
\hline-2.0 & 1.1 & 0.594 & 1987.315 & 25-Apr-87 & \\
\hline-8.5 & 4.5 & 0.767 & 1987.488 & 27-Jun-87 & \\
\hline-2.0 & 1.8 & 0.928 & 1987.649 & 25-Aug-87 & -2.2 \\
\hline-6.5 & 1.8 & 1.306 & 1988.027 & 10-Jan-88 & -5.0 \\
\hline-3.2 & 2.3 & 1.612 & 1988.333 & 1-May-88 & -2.0 \\
\hline-0.2 & 2.1 & 1.839 & 1988.560 & 23-Jul-88 & -0.1 \\
\hline 4.7 & 3.7 & 2.271 & 1988.992 & 28-Dec-88 & 2.1 \\
\hline-0.2 & 4.0 & 2.474 & 1989.195 & 12-Mar-89 & -0.1 \\
\hline-1.8 & 1.9 & 2.701 & 1989.422 & 3-Jun-89 & -0.7 \\
\hline-0.3 & 2.5 & 2.835 & 1989.556 & 22-Jul-89 & -0.1 \\
\hline 1.8 & 1.4 & 3.065 & 1989.786 & 14-Oct-89 & 0.6 \\
\hline 3.3 & 1.6 & 3.241 & 1989.962 & 17-Dec-89 & 1.0 \\
\hline 0.5 & 1.5 & 3.411 & 1990.132 & 17-Feb-90 & 0.1 \\
\hline-2.6 & 0.8 & 3.602 & 1990.323 & 28-Apr-90 & -0.7 \\
\hline-3.0 & 0.8 & 3.737 & 1990.458 & 16-Jun-90 & -0.8 \\
\hline 5.2 & 0.9 & 3.871 & 1990.592 & 4-Aug-90 & 1.3 \\
\hline 7.0 & 0.8 & 4.043 & 1990.764 & 6-Oct-90 & 1.7 \\
\hline 10.1 & 1.0 & 4.123 & 1990.844 & 4-Nov-90 & 2.4 \\
\hline 5.4 & 1.7 & 4.254 & 1990.975 & 22-Dec-90 & 1.3 \\
\hline 6.2 & 0.8 & 4.446 & 1991.167 & 2-Mar-91 & 1.4 \\
\hline-0.2 & 0.7 & 4.580 & 1991.301 & 20-Apr-91 & 0.0 \\
\hline 2.2 & 1.1 & 4.753 & 1991.474 & 22-Jun-91 & 0.5 \\
\hline 8.8 & 1.6 & 4.906 & 1991.627 & 17-Aug-91 & 1.8 \\
\hline 12.5 & 2.1 & 5.098 & 1991.819 & $26-$-oct-91 & 2.5 \\
\hline 10.5 & 0.8 & 5.328 & 1992.049 & 18-Jan-92 & 2.0 \\
\hline 2.3 & 2.0 & 5.596 & 1992.317 & 25-Apr-92 & 0.4 \\
\hline 5.3 & 2.0 & 5.787 & 1992.508 & 4-Jul-92 & 0.9 \\
\hline 10.0 & 2.0 & 6.036 & 1992.757 & 3-Oct-92 & 1.7 \\
\hline 4.0 & 2.2 & 6.342 & 1993.063 & 23-Jan-93 & 0.6 \\
\hline 4.0 & 1.8 & 6.572 & 1993.293 & 17-Apr-93 & 0.6 \\
\hline 8.7 & 1.5 & 6.764 & 1993.485 & 26-Jun-93 & 1.3 \\
\hline 20.2 & 1.1 & 6.975 & 1993.696 & $11-$ Sep-93 & 2.9 \\
\hline 12.1 & 2.0 & 7.361 & 1994.082 & 30-Jan-94 & 1.6 \\
\hline 6.1 & 2.6 & 7.569 & 1994.290 & 16-Apr-94 & 0.8 \\
\hline 7.6 & 2.1 & 7.802 & 1994.523 & 10-Jul-94 & 1.0 \\
\hline 15.5 & 3.0 & 8.071 & 1994.792 & 16-Oct-94 & 1.9 \\
\hline 10.1 & 2.7 & 8.252 & 1994.973 & 21-Dec-94 & 1.2 \\
\hline 5.4 & 1.0 & 8.452 & 1995.173 & 4-Mar-95 & 0.6 \\
\hline 9.7 & 0.8 & 8.663 & 1995.384 & 20-May-95 & 1.1 \\
\hline 17.5 & 1.7 & 9.180 & 1995.901 & $25-N o v-95$ & 1.9 \\
\hline 18.9 & 5.4 & 9.372 & 1996.093 & 3-Feb-96 & 2.0 \\
\hline 13.4 & 2.3 & 9.659 & 1996.380 & 18-May-96 & 1.4 \\
\hline 20.8 & 2.2 & 9.869 & 1996.590 & 3-Aug-96 & 2.1 \\
\hline 15.8 & 1.8 & 10.137 & 1996.858 & 9-Nov-96 & 1.6 \\
\hline
\end{tabular}




$\begin{array}{llllcl}16.6 & 5.8 & 10.389 & 1997.110 & \text { 9-Feb-97 } & 1.6 \\ 17.2 & 2.3 & 10.616 & 1997.337 & \text { 3-May-97 } & 1.6 \\ 15.3 & 1.7 & 11.000 & 1997.721 & \text { 20-Sep-97 } & 1.4 \\ 15.6 & 1.8 & 11.290 & 1998.011 & \text { 4-Jan-98 } & 1.4 \\ 18.3 & 1.5 & 11.613 & 1998.334 & \text { 2-May-98 } & 1.6 \\ 12.4 & 1.3 & 12.054 & 1998.775 & 10-\text { Oct-98 } & 1.0 \\ 16.3 & 1.0 & 12.323 & 1999.044 & 16-\text { Jan-99 } & 1.3 \\ 16.4 & 3.8 & 12.534 & 1999.255 & \text { 3-Apr-99 } & 1.3 \\ 12.4 & 0.8 & 12.764 & 1999.485 & 26 \text {-Jun-99 } & 1.0 \\ 14.6 & 3.9 & 13.071 & 1999.792 & \text { 16-Oct-99 } & 1.1 \\ 14.5 & 3.3 & 13.304 & 2000.025 & \text { 9-Jan-00 } & 1.0 \\ 15.0 & 1.1 & 13.590 & 2000.311 & \text { 23-Apr-00 } & 1.1 \\ 17.2 & 3.5 & 13.894 & 2000.615 & \text { 12-Aug-00 } & 1.2 \\ 14.4 & 4.2 & 14.183 & 2000.904 & \text { 26-Nov-00 } & 1.0\end{array}$


SF-22 Roberta Drive in Woodside San Andreas Fault

\begin{tabular}{|c|c|c|c|c|c|}
\hline $\begin{array}{l}\text { movement } \\
(\mathrm{mm})\end{array}$ & std. dev. & \# of years & year & date & $\begin{array}{c}\mathrm{mm} / \mathrm{yr} \text {-simple } \\
\text { average }\end{array}$ \\
\hline 0.0 & 1.1 & 0.000 & 1989.844 & 4-Nov-89 & \\
\hline 1.3 & 1.8 & 0.035 & 1989.879 & 17-Nov-89 & \\
\hline 1.2 & 0.8 & 0.115 & 1989.959 & 16-Dec-89 & \\
\hline 2.1 & 0.9 & 0.211 & 1990.055 & 20-Jan-90 & \\
\hline 0.9 & 1.8 & 0.249 & 1990.093 & $3-F e b-90$ & \\
\hline 3.2 & 0.3 & 0.326 & 1990.170 & 3-Mar-90 & \\
\hline 4.0 & 0.6 & 0.444 & 1990.288 & $15-A p r-90$ & \\
\hline 4.0 & 0.5 & 0.457 & 1990.301 & 20-Apr-90 & \\
\hline 2.3 & 2.5 & 0.561 & 1990.405 & 28-May-90 & \\
\hline 3.7 & 0.8 & 0.638 & 1990.482 & 25-Jun-90 & \\
\hline 1.4 & 0.5 & 0.729 & 1990.573 & 28-Jul-90 & \\
\hline 1.5 & 1.4 & 0.838 & 1990.682 & 6-Sep-90 & \\
\hline 0.0 & 1.8 & 0.953 & 1990.797 & 18-Oct-90 & 0.0 \\
\hline-1.3 & 1.4 & 1.208 & 1991.052 & 19-Jan-91 & -1.1 \\
\hline 0.3 & 1.7 & 1.400 & 1991.244 & 30-Mar-91 & 0.2 \\
\hline 3.5 & 2.2 & 1.553 & 1991.397 & 25-May-91 & 2.3 \\
\hline 4.6 & 1.6 & 1.649 & 1991.493 & 29-Jun-91 & 2.8 \\
\hline 4.2 & 0.9 & 1.822 & 1991.666 & 31-Aug-91 & 2.3 \\
\hline 3.1 & 2.4 & 1.956 & 1991.800 & 19-Oct-91 & 1.6 \\
\hline 3.3 & 1.0 & 2.129 & 1991.973 & 21-Dec-91 & 1.6 \\
\hline 3.0 & 2.1 & 2.339 & 1992.183 & 7-Mar-92 & 1.3 \\
\hline 5.3 & 1.6 & 2.533 & 1992.377 & 17-May-92 & 2.1 \\
\hline 6.5 & 1.6 & 2.722 & 1992.566 & 25-Jul-92 & 2.4 \\
\hline 4.0 & 0.9 & 2.894 & 1992.738 & $26-$ Sep-92 & 1.4 \\
\hline 3.3 & 1.4 & 3.066 & 1992.910 & 28-Nov-92 & 1.1 \\
\hline 2.8 & 1.0 & 3.277 & 1993.121 & 13-Feb-93 & 0.9 \\
\hline 2.4 & 0.5 & 3.583 & 1993.427 & 5-Jun-93 & 0.7 \\
\hline 1.4 & 0.8 & 3.871 & 1993.715 & 18-Sep-93 & 0.4 \\
\hline 2.7 & 0.4 & 4.219 & 1994.063 & 23-Jan-94 & 0.6 \\
\hline 3.5 & 1.1 & 4.351 & 1994.195 & 12-Mar-94 & 0.8 \\
\hline 5.3 & 0.6 & 4.564 & 1994.408 & 29-May-94 & 1.2 \\
\hline 7.1 & 0.8 & 4.753 & 1994.597 & 6-Aug-94 & 1.5 \\
\hline 3.7 & 0.4 & 4.983 & 1994.827 & 29-Oct-94 & 0.7 \\
\hline 3.9 & 0.7 & 5.194 & 1995.038 & 14-Jan-95 & 0.8 \\
\hline 2.6 & 0.9 & 5.348 & 1995.192 & 11-Mar-95 & 0.5 \\
\hline 3.1 & 1.0 & 5.578 & 1995.422 & 3-Jun-95 & 0.6 \\
\hline 6.9 & 1.6 & 5.926 & 1995.770 & 8-Oct-95 & 1.2 \\
\hline 6.9 & 1.1 & 5.942 & 1995.786 & $14-$ Oct-95 & 1.2 \\
\hline 4.6 & 1.6 & 6.175 & 1996.019 & 7-Jan-96 & 0.7 \\
\hline 3.3 & 2.7 & 6.440 & 1996.284 & 13-Apr-96 & 0.5 \\
\hline 5.3 & 1.3 & 6.708 & 1996.552 & 20-Jul-96 & 0.8 \\
\hline 3.4 & 0.3 & 6.995 & 1996.839 & 2-Nov-96 & 0.5 \\
\hline 2.2 & 0.3 & 7.282 & 1997.126 & 15-Feb-97 & 0.3 \\
\hline 5.2 & 0.2 & 7.496 & 1997.340 & 4-May-97 & 0.7 \\
\hline 4.4 & 0.5 & 7.764 & 1997.608 & 10-Aug-97 & 0.6 \\
\hline 3.1 & 0.5 & 8.011 & 1997.855 & 8-Nov-97 & 0.4 \\
\hline
\end{tabular}




$\begin{array}{llllll}2.0 & 0.4 & 8.318 & 1998.162 & 28 \text {-Feb-98 } & 0.2 \\ 4.1 & 1.1 & 8.548 & 1998.392 & 23-\text { May-98 } & 0.5 \\ 4.3 & 0.5 & 8.797 & 1998.641 & 22-\text { Aug-98 } & 0.5 \\ 5.6 & 0.8 & 9.123 & 1998.967 & 19-\text { Dec-98 } & 0.6 \\ 4.5 & 1.3 & 9.353 & 1999.197 & 13-\text { Mar-99 } & 0.5 \\ 5.3 & 0.6 & 9.605 & 1999.449 & 13-J u n-99 & 0.6 \\ 4.9 & 0.3 & 9.951 & 1999.795 & 17-\text { Oct-99 } & 0.5 \\ 6.0 & 0.6 & 10.216 & 2000.060 & 22-\text { Jan-00 } & 0.6 \\ 6.0 & 0.4 & 10.525 & 2000.369 & 14-\text { May-00 } & 0.6 \\ 6.2 & 1.1 & 10.771 & 2000.615 & 12-\text { Aug-00 } & 0.6 \\ 7.2 & 0.9 & 11.060 & 2000.904 & 26 \text {-Nov-00 } & 0.7\end{array}$


SF-23 Cannon Road near San Juan Bautista San Andreas Fault

\begin{tabular}{|c|c|c|c|c|c|}
\hline $\begin{array}{l}\text { movement } \\
(\mathrm{mm})\end{array}$ & std. dev. & \# of years & year & date & $\begin{array}{c}\mathrm{mm} / \mathrm{yr} \text {-simple } \\
\text { average }\end{array}$ \\
\hline 0.0 & 0.6 & 0.000 & 1989.882 & 18-Nov-89 & \\
\hline-0.1 & 0.5 & 0.077 & 1989.959 & 16-Dec-89 & \\
\hline 1.0 & 1.3 & 0.173 & 1990.055 & 20-Jan-90 & \\
\hline-0.5 & 1.1 & 0.288 & 1990.170 & 3-Mar-90 & \\
\hline 0.8 & 0.5 & 0.406 & 1990.288 & 15-Apr-90 & \\
\hline 0.5 & 0.6 & 0.523 & 1990.405 & 28-May-90 & \\
\hline-0.4 & 0.8 & 0.671 & 1990.553 & 21-Jul-90 & \\
\hline 0.4 & 0.5 & 0.786 & 1990.668 & 1-Sep-90 & \\
\hline 2.0 & 1.3 & 0.902 & 1990.784 & 13-Oct-90 & \\
\hline 0.6 & 1.1 & 1.055 & 1990.937 & 8-Dec-90 & 0.6 \\
\hline 1.8 & 1.1 & 1.304 & 1991.186 & 9-Mar-91 & 1.4 \\
\hline 0.8 & 0.7 & 1.433 & 1991.315 & 25-Apr-91 & 0.6 \\
\hline-2.3 & 0.7 & 1.614 & 1991.496 & 30-Jun-91 & -1.4 \\
\hline-0.9 & 0.8 & 1.784 & 1991.666 & 31-Aug-91 & -0.5 \\
\hline 0.3 & 0.8 & 1.899 & 1991.781 & 12-Oct-91 & 0.2 \\
\hline 1.5 & 1.1 & 2.074 & 1991.956 & 15-Dec-91 & 0.7 \\
\hline 1.1 & 0.3 & 2.339 & 1992.221 & 21-Mar-92 & 0.5 \\
\hline 1.5 & 0.5 & 2.492 & 1992.374 & 16-May-92 & 0.6 \\
\hline 0.9 & 1.0 & 2.667 & 1992.549 & 19-Jul-92 & 0.3 \\
\hline-1.4 & 1.2 & 2.837 & 1992.719 & 19-Sep-92 & -0.5 \\
\hline-2.6 & 0.6 & 3.047 & 1992.929 & 5-Dec-92 & -0.9 \\
\hline 0.5 & 0.6 & 3.258 & 1993.140 & 20-Feb-93 & 0.2 \\
\hline 0.5 & 1.8 & 3.450 & 1993.332 & 1-May-93 & 0.1 \\
\hline 1.0 & 0.6 & 3.584 & 1993.466 & 19-Jun-93 & 0.3 \\
\hline-0.3 & 0.4 & 3.795 & 1993.677 & 4-Sep-93 & -0.1 \\
\hline-2.9 & 0.9 & 4.008 & 1993.890 & 21-Nov-93 & -0.7 \\
\hline 0.8 & 0.7 & 4.274 & 1994.156 & $26-F e b-94$ & 0.2 \\
\hline 0.8 & 2.4 & 4.428 & 1994.310 & 23-Apr-94 & 0.2 \\
\hline-5.0 & 1.1 & 4.658 & 1994.540 & 16-Jul-94 & -1.1 \\
\hline-2.0 & 0.4 & 4.945 & 1994.827 & 29-Oct-94 & -0.4 \\
\hline-1.6 & 0.1 & 5.156 & 1995.038 & 14-Jan-95 & -0.3 \\
\hline-0.6 & 1.0 & 5.310 & 1995.192 & 11-Mar-95 & -0.1 \\
\hline 0.7 & 0.8 & 5.540 & 1995.422 & 3-Jun-95 & 0.1 \\
\hline-2.6 & 1.5 & 5.828 & 1995.710 & 16-Sep-95 & -0.4 \\
\hline-3.8 & 0.7 & 6.000 & 1995.882 & 18-Nov-95 & -0.6 \\
\hline 2.2 & 1.7 & 6.402 & 1996.284 & 13-Apr-96 & 0.3 \\
\hline 2.2 & 2.3 & 6.670 & 1996.552 & 20-Jul-96 & 0.3 \\
\hline 0.7 & 0.5 & 6.957 & 1996.839 & 2-Nov-96 & 0.1 \\
\hline 3.7 & 1.1 & 7.244 & 1997.126 & $15-F e b-97$ & 0.5 \\
\hline 2.4 & 0.4 & 7.458 & 1997.340 & 4-May-97 & 0.3 \\
\hline 2.4 & 0.6 & 7.726 & 1997.608 & 10-Aug-97 & 0.3 \\
\hline 2.4 & 0.4 & 7.992 & 1997.874 & $15-N o v-97$ & 0.3 \\
\hline 1.6 & 0.5 & 8.241 & 1998.123 & 14-Feb-98 & 0.2 \\
\hline
\end{tabular}


SF-24 Camellia Drive in Fremont Hayward Fault 66.29 km

\begin{tabular}{|c|c|c|c|c|c|}
\hline $\begin{array}{l}\text { movement } \\
(\mathrm{mm})\end{array}$ & std. dev. & \# of years & year & date & $\begin{array}{c}\mathrm{mm} / \mathrm{yr} \text {-simple } \\
\text { average }\end{array}$ \\
\hline 0.0 & 0.6 & 0.000 & 1990.115 & 11-Feb-90 & \\
\hline 0.5 & 0.7 & 0.134 & 1990.249 & 1-Apr-90 & \\
\hline-0.4 & 1.0 & 0.263 & 1990.378 & 18-Мау-90 & \\
\hline-0.3 & 0.8 & 0.362 & 1990.477 & 23-Jun-90 & \\
\hline-1.0 & 0.7 & 0.463 & 1990.578 & 30-Jul-90 & \\
\hline-1.2 & 0.6 & 0.595 & 1990.710 & 16-Sep-90 & \\
\hline-0.2 & 0.5 & 0.710 & 1990.825 & 28-Oct-90 & \\
\hline 0.2 & 0.7 & 0.959 & 1991.074 & 27-Jan-91 & 0.2 \\
\hline-0.9 & 0.8 & 1.126 & 1991.241 & 29-Mar-91 & -0.8 \\
\hline 0.0 & 0.5 & 1.181 & 1991.296 & 18-Apr-91 & 0.0 \\
\hline-1.6 & 0.3 & 1.244 & 1991.359 & 11-May-91 & -1.3 \\
\hline-0.8 & 0.3 & 1.436 & 1991.551 & 20-Jul-91 & -0.6 \\
\hline-2.7 & 0.5 & 1.627 & 1991.742 & 28-Sep-91 & -1.7 \\
\hline-1.7 & 0.5 & 1.784 & 1991.899 & 24-Nov-91 & -1.0 \\
\hline-1.5 & 1.4 & 1.937 & 1992.052 & 19-Jan-92 & -0.8 \\
\hline-0.9 & 0.7 & 2.147 & 1992.262 & 5-Apr-92 & -0.4 \\
\hline-1.5 & 0.9 & 2.317 & 1992.432 & 6-Jun-92 & -0.6 \\
\hline-4.0 & 1.4 & 2.489 & 1992.604 & 8-Aug-92 & -1.6 \\
\hline-2.1 & 1.3 & 2.661 & 1992.776 & 10-Oct-92 & -0.8 \\
\hline-2.6 & 1.1 & 2.808 & 1992.923 & 3-Dec-92 & -0.9 \\
\hline-2.4 & 1.1 & 3.008 & 1993.123 & 14-Feb-93 & -0.8 \\
\hline-1.8 & 2.2 & 3.159 & 1993.274 & 10-Apr-93 & -0.6 \\
\hline-3.9 & 0.8 & 3.255 & 1993.370 & 15-May-93 & -1.2 \\
\hline-3.7 & 2.0 & 3.523 & 1993.638 & 21-Aug-93 & -1.1 \\
\hline-3.4 & 1.3 & 3.658 & 1993.773 & 9-Oct-93 & -0.9 \\
\hline-2.1 & 0.8 & 3.847 & 1993.962 & 17-Dec-93 & -0.5 \\
\hline-2.4 & 0.7 & 4.044 & 1994.159 & 27-Feb-94 & -0.6 \\
\hline-3.0 & 1.4 & 4.197 & 1994.312 & 24-Apr-94 & -0.7 \\
\hline-2.8 & 0.8 & 4.427 & 1994.542 & 17-Jul-94 & -0.6 \\
\hline-1.2 & 0.7 & 4.655 & 1994.770 & 8-Oct-94 & -0.3 \\
\hline-1.5 & 0.6 & 4.847 & 1994.962 & 17-Dec-94 & -0.3 \\
\hline-1.5 & 0.8 & 5.038 & 1995.153 & 25-Feb-95 & -0.3 \\
\hline 1.9 & 0.5 & 5.211 & 1995.326 & 29-Apr-95 & 0.4 \\
\hline-0.8 & 1.0 & 5.367 & 1995.482 & 25-Jun-95 & -0.1 \\
\hline 1.0 & 0.5 & 5.537 & 1995.652 & 26-Aug-95 & 0.2 \\
\hline 2.4 & 0.5 & 5.575 & 1995.690 & 9-Sep-95 & 0.4 \\
\hline 4.1 & 1.4 & 5.693 & 1995.808 & 22-Oct-95 & 0.7 \\
\hline 3.3 & 0.3 & 5.844 & 1995.959 & 16-Dec-95 & 0.6 \\
\hline 21.3 & 2.2 & 6.016 & 1996.131 & 17-Feb-96 & 3.5 \\
\hline 24.5 & 1.2 & 6.038 & 1996.153 & 25-Feb-96 & 4.1 \\
\hline 23.3 & 4.0 & 6.076 & 1996.191 & 10-Mar-96 & 3.8 \\
\hline 23.4 & 1.3 & 6.246 & 1996.361 & 11-May-96 & 3.7 \\
\hline 24.7 & 2.9 & 6.418 & 1996.533 & 13-Jul-96 & 3.8 \\
\hline 23.3 & 1.9 & 6.527 & 1996.642 & 22-Aug-96 & 3.6 \\
\hline
\end{tabular}




\begin{tabular}{|c|c|c|c|c|c|c|}
\hline 24.4 & 1.1 & 6.686 & 1996.801 & 19-Oct-96 & 3.6 & \\
\hline 24.1 & 0.3 & 6.839 & 1996.954 & 14-Dec-96 & 3.5 & \\
\hline 23.5 & 1.0 & 6.992 & 1997.107 & 8-Feb-97 & 3.4 & 2.3 \\
\hline 24.2 & 0.6 & 7.164 & 1997.279 & 12-Apr-97 & 3.4 & 2.5 \\
\hline 23.7 & 1.0 & 7.340 & 1997.455 & 15-Jun-97 & 3.2 & 1.8 \\
\hline 25.0 & 0.7 & 7.532 & 1997.647 & 24-Aug-97 & 3.3 & 2.4 \\
\hline 23.8 & 0.8 & 7.682 & 1997.797 & 18-Oct-97 & 3.1 & 1 \\
\hline 24.6 & 1.1 & 7.874 & 1997.989 & 27-Dec-97 & 3.1 & 1.8 \\
\hline 23.4 & 1.2 & 8.047 & 1998.162 & 28-Feb-98 & 2.9 & 1.0 \\
\hline 24.0 & 0.9 & 8.200 & 1998.315 & 25-Apr-98 & 2.9 & 1.2 \\
\hline 23.5 & 1.2 & 8.373 & 1998.488 & 27-Jun-98 & 2.8 & 0.9 \\
\hline 26.3 & 2.8 & 8.570 & 1998.685 & 7-Sep-98 & 3.1 & 2 \\
\hline 25.5 & 0.5 & 8.775 & 1998.890 & 21-Nov-98 & 2.9 & 1. \\
\hline 25.0 & 1.1 & 8.967 & 1999.082 & 30-Jan-99 & 2.8 & 1.3 \\
\hline 26.1 & 0.9 & 9.104 & 1999.219 & 21-Mar-99 & 2.9 & 1 \\
\hline 26.5 & 1.3 & 9.274 & 1999.389 & 22-May-99 & 2.9 & 1.6 \\
\hline 28.0 & 0.5 & 9.581 & 1999.696 & 11-Sep-99 & 2.9 & 1.9 \\
\hline 31.3 & 1.5 & 9.734 & 1999.849 & 6-Nov-99 & 3.2 & 2.7 \\
\hline 31.2 & 0.4 & 9.964 & 2000.079 & 29-Jan-00 & 3.1 & 2 \\
\hline 30.3 & 0.7 & 10.117 & 2000.232 & 25-Mar-00 & 3.0 & 2. \\
\hline 31.4 & 1.7 & 10.273 & 2000.388 & 21-May-00 & 3.1 & 2.4 \\
\hline 32.1 & 0.6 & 10.462 & 2000.577 & 29-Jul-00 & 3.1 & 2. \\
\hline 33.8 & 0.8 & 10.691 & 2000.806 & 21-Oct-00 & 3.2 & 2.7 \\
\hline 35.2 & 0.6 & 10.825 & 2000.940 & 9-Dec-00 & 3.3 & 2. \\
\hline 35.2 & 0.3 & 10.997 & 2001.112 & 10-Feb-01 & 3.2 & 2.8 \\
\hline
\end{tabular}


SF-25 Mission Vineyard Road near San Juan Bautista San Andreas Fault

\begin{tabular}{|c|c|c|c|c|c|}
\hline $\begin{array}{l}\text { movement } \\
\quad(\mathrm{mm})\end{array}$ & std. dev. & \# of years & year & date & $\begin{array}{c}\mathrm{mm} / \mathrm{yr} \text {-simple } \\
\text { average }\end{array}$ \\
\hline 0.0 & 0.4 & 0.000 & 1990.553 & 21-Jul-90 & \\
\hline 5.6 & 1.1 & 0.115 & 1990.668 & 1-Sep-90 & \\
\hline 6.4 & 2.4 & 0.231 & 1990.784 & 13 -Oct-90 & \\
\hline 9.0 & 1.9 & 0.384 & 1990.937 & 8-Dec-90 & \\
\hline 9.8 & 1.5 & 0.633 & 1991.186 & 9-Mar-91 & \\
\hline 11.8 & 1.1 & 0.762 & 1991.315 & 25-Apr-91 & \\
\hline 14.6 & 0.9 & 0.979 & 1991.532 & 13-Jul-91 & 14.9 \\
\hline 27.3 & 1.4 & 1.228 & 1991.781 & 12-Oct-91 & 22.2 \\
\hline 20.3 & 0.5 & 1.403 & 1991.956 & 15-Dec-91 & 14.5 \\
\hline 22.2 & 0.6 & 1.668 & 1992.221 & 21-Mar-92 & 13.3 \\
\hline 29.1 & 1.3 & 1.821 & 1992.374 & 16-May-92 & 16.0 \\
\hline 35.3 & 1.3 & 1.996 & 1992.549 & 19-Jul-92 & 17.7 \\
\hline 31.3 & 2.1 & 2.166 & 1992.719 & 19-Sep-92 & 14.5 \\
\hline 31.8 & 2.7 & 2.376 & 1992.929 & 5-Dec-92 & 13.4 \\
\hline 36.1 & 2.8 & 2.587 & 1993.140 & 20-Feb-93 & 14.0 \\
\hline 44.0 & 3.2 & 2.779 & 1993.332 & 1-May-93 & 15.8 \\
\hline 48.7 & 1.3 & 2.913 & 1993.466 & 19-Jun-93 & 16.7 \\
\hline 50.2 & 1.1 & 3.124 & 1993.677 & 4-Sep-93 & 16.1 \\
\hline 49.0 & 0.6 & 3.337 & 1993.890 & 21-Nov-93 & 14.7 \\
\hline 51.8 & 2.7 & 3.603 & 1994.156 & 26-Feb-94 & 14.4 \\
\hline 52.2 & 3.9 & 3.757 & 1994.310 & 23-Apr-94 & 13.9 \\
\hline 60.9 & 1.9 & 3.987 & 1994.540 & 16-Jul-94 & 15.3 \\
\hline 57.4 & 2.8 & 4.313 & 1994.866 & 12-Nov-94 & 13.3 \\
\hline 58.3 & 0.5 & 4.562 & 1995.115 & 11-Feb-95 & 12.8 \\
\hline 62.8 & 1.1 & 4.754 & 1995.307 & 22-Apr-95 & 13.2 \\
\hline 64.8 & 0.8 & 4.776 & 1995.329 & 30-Apr-95 & 13.6 \\
\hline 68.2 & 0.7 & 4.984 & 1995.537 & 15-Jul-95 & 13.7 \\
\hline 73.7 & 1.4 & 5.157 & 1995.710 & 16-Sep-95 & 14.3 \\
\hline 71.5 & 1.1 & 5.329 & 1995.882 & 18-Nov-95 & 13.4 \\
\hline 71.5 & 1.9 & 5.444 & 1995.997 & 30-Dec-95 & 13.1 \\
\hline 71.4 & 3.3 & 5.636 & 1996.189 & 9-Mar-96 & 12.7 \\
\hline 71.6 & 4.0 & 5.772 & 1996.325 & 28-Apr-96 & 12.4 \\
\hline 79.7 & 1.8 & 5.846 & 1996.399 & 25-May-96 & 13.6 \\
\hline 78.5 & 0.9 & 6.018 & 1996.571 & 27-Jul-96 & 13.0 \\
\hline 79.2 & 1.0 & 6.190 & 1996.743 & 28-Sep-96 & 12.8 \\
\hline 77.8 & 1.9 & 6.324 & 1996.877 & 16-Nov-96 & 12.3 \\
\hline 76.2 & 0.8 & 6.496 & 1997.049 & 18-Jan-97 & 11.7 \\
\hline 80.2 & 1.9 & 6.669 & 1997.222 & 22-Mar-97 & 12.0 \\
\hline 78.9 & 0.4 & 6.803 & 1997.356 & 10-May-97 & 11.6 \\
\hline 81.8 & 0.9 & 6.976 & 1997.529 & 12-Jul-97 & 11.7 \\
\hline 88.3 & 1.1 & 7.170 & 1997.723 & 21-Sep-97 & 12.3 \\
\hline 84.5 & 2.6 & 7.321 & 1997.874 & 15-Nov-97 & 11.5 \\
\hline 81.6 & 1.1 & 7.532 & 1998.085 & 31-Jan-98 & 10.8 \\
\hline 83.9 & 0.6 & 7.688 & 1998.241 & 29-Mar-98 & 10.9 \\
\hline 83.6 & 1.2 & 7.858 & 1998.411 & 30-May-98 & 10.6 \\
\hline 82.3 & 0.3 & 8.011 & 1998.564 & 25-Jul-98 & 10.3 \\
\hline
\end{tabular}




$\begin{array}{cccccc}87.2 & 0.7 & 8.069 & 1998.622 & \text { 15-Aug-98 } & 10.8 \\ 92.7 & 1.1 & 8.280 & 1998.833 & \text { 31-Oct-98 } & 11.2 \\ 96.0 & 0.6 & 8.472 & 1999.025 & \text { 9-Jan-99 } & 11.3 \\ 96.1 & 0.8 & 8.628 & 1999.181 & \text { 7-Mar-99 } & 11.1 \\ 98.3 & 1.1 & 8.798 & 1999.351 & \text { 8-May-99 } & 11.2 \\ 107.8 & 1.3 & 8.970 & 1999.523 & 10-J u l-99 & 12.0 \\ 106.2 & 2.4 & 9.181 & 1999.734 & 25-\text { Sep-99 } & 11.6 \\ 106.1 & 3.3 & 9.354 & 1999.907 & 27-\text { Nov-99 } & 11.3 \\ 109.4 & 1.4 & 9.545 & 2000.098 & \text { 5-Feb-00 } & 11.5 \\ 108.6 & 2.1 & 9.717 & 2000.270 & \text { 8-Apr-00 } & 11.2 \\ 111.4 & 3.5 & 9.890 & 2000.443 & \text { 10-Jun-00 } & 11.3 \\ 116.1 & 1.3 & 10.081 & 2000.634 & \text { 19-Aug-00 } & 11.5 \\ 118.9 & 2.6 & 10.234 & 2000.787 & \text { 14-Oct-00 } & 11.6 \\ 118.1 & 1.3 & 10.406 & 2000.959 & \text { 16-Dec-00 } & 11.3 \\ 118.3 & 1.6 & 10.600 & 2001.153 & \text { 25-Feb-01 } & 11.2\end{array}$


SF-26 West Commercial Ave in Willits Maacama Fault

\begin{tabular}{|c|c|c|c|c|c|}
\hline $\begin{array}{l}\text { movement } \\
(\mathrm{mm})\end{array}$ & std. dev. & \# of years & year & date & $\begin{array}{c}\mathrm{mm} / \mathrm{yr} \text {-simple } \\
\text { average }\end{array}$ \\
\hline 0.0 & 1.3 & 0.000 & 1991.871 & 14-Nov-91 & \\
\hline 3.5 & 1.3 & 0.430 & 1992.301 & 19-Apr-92 & \\
\hline 6.3 & 1.4 & 0.580 & 1992.451 & 13-Jun-92 & \\
\hline 3.8 & 1.1 & 0.793 & 1992.664 & 30-Aug-92 & \\
\hline 6.0 & 2.2 & 0.976 & 1992.847 & 5-Nov-92 & 6.1 \\
\hline 7.7 & 3.1 & 1.184 & 1993.055 & 20-Jan-93 & 6.5 \\
\hline 7.9 & 2.0 & 1.518 & 1993.389 & 22-May-93 & 5.2 \\
\hline 8.1 & 2.7 & 1.748 & 1993.619 & 14-Aug-93 & 4.6 \\
\hline 7.8 & 3.0 & 1.940 & 1993.811 & 23-Oct-93 & 4.0 \\
\hline 11.3 & 1.1 & 2.151 & 1994.022 & 8-Jan-94 & 5.3 \\
\hline 16.7 & 2.1 & 2.343 & 1994.214 & 19-Mar-94 & 7.1 \\
\hline 18.8 & 1.7 & 2.480 & 1994.351 & 8-May-94 & 7.6 \\
\hline 19.8 & 0.8 & 2.650 & 1994.521 & 9-Jul-94 & 7.5 \\
\hline 20.8 & 1.5 & 2.822 & 1994.693 & 10-Sep-94 & 7.4 \\
\hline 24.3 & 1.3 & 3.014 & 1994.885 & 19-Nov-94 & 8.1 \\
\hline 26.5 & 1.0 & 3.187 & 1995.058 & 21-Jan-95 & 8.3 \\
\hline 27.6 & 1.6 & 3.359 & 1995.230 & 25-Mar-95 & 8.2 \\
\hline 25.4 & 1.1 & 3.570 & 1995.441 & 10-Jun-95 & 7.1 \\
\hline 26.5 & 2.4 & 3.743 & 1995.614 & 12-Aug-95 & 7.1 \\
\hline 25.4 & 1.0 & 3.956 & 1995.827 & 29-Oct-95 & 6.4 \\
\hline 29.4 & 2.6 & 4.184 & 1996.055 & 20-Jan-96 & 7.0 \\
\hline 30.0 & 4.4 & 4.375 & 1996.246 & 30-Mar-96 & 6.9 \\
\hline 30.2 & 4.1 & 4.585 & 1996.456 & 15-Jun-96 & 6.6 \\
\hline 29.2 & 1.0 & 4.796 & 1996.667 & 31-Aug-96 & 6.1 \\
\hline 32.0 & 2.1 & 4.971 & 1996.842 & 3-Nov-96 & 6.4 \\
\hline 40.2 & 1.4 & 5.217 & 1997.088 & 1-Feb-97 & 7.7 \\
\hline 37.9 & 0.7 & 5.389 & 1997.260 & 5-Apr-97 & 7.0 \\
\hline 39.9 & 1.2 & 5.581 & 1997.452 & 14-Jun-97 & 7.1 \\
\hline 37.6 & 0.4 & 5.734 & 1997.605 & 9-Aug-97 & 6.6 \\
\hline 39.8 & 1.2 & 5.945 & 1997.816 & $25-O c t-97$ & 6.7 \\
\hline 44.2 & 2.1 & 6.099 & 1997.970 & 20-Dec-97 & 7.2 \\
\hline 45.5 & 0.9 & 6.310 & 1998.181 & 7-Mar-98 & 7.2 \\
\hline 45.6 & 1.1 & 6.482 & 1998.353 & 9-May-98 & 7.0 \\
\hline 43.5 & 0.4 & 6.655 & 1998.526 & 11-Jul-98 & 6.5 \\
\hline 44.2 & 1.2 & 6.847 & 1998.718 & 19-Sep-98 & 6.5 \\
\hline 46.1 & 0.9 & 7.022 & 1998.893 & 22-Nov-98 & 6.6 \\
\hline 49.2 & 2.0 & 7.195 & 1999.066 & 24-Jan-99 & 6.8 \\
\hline 50.9 & 1.0 & 7.345 & 1999.216 & 20-Mar-99 & 6.9 \\
\hline 50.9 & 1.0 & 7.499 & 1999.370 & 15-May-99 & 6.8 \\
\hline 48.7 & 3.3 & 7.693 & 1999.564 & 25-Jul-99 & 6.3 \\
\hline 48.8 & 3.4 & 7.904 & 1999.775 & 10-Oct-99 & 6.2 \\
\hline 50.9 & 1.4 & 8.074 & 1999.945 & 11-Dec-99 & 6.3 \\
\hline 53.5 & 2.7 & 8.323 & 2000.194 & 11-Mar-00 & 6.4 \\
\hline 54.0 & 2.8 & 8.495 & 2000.366 & 13-May-00 & 6.4 \\
\hline 56.5 & 1.4 & 8.880 & 2000.751 & $1-O c t-00$ & 6.4 \\
\hline 57.8 & 3.0 & 9.050 & 2000.921 & 2-Dec-00 & 6.4 \\
\hline 61.2 & 2.2 & 9.280 & 2001.151 & 24-Feb-01 & 6.6 \\
\hline
\end{tabular}




\begin{tabular}{|c|c|c|c|c|c|c|}
\hline $\begin{array}{l}\text { movement } \\
(\mathrm{mm})\end{array}$ & std. dev. & \# of years & year & date & $\begin{array}{c}\mathrm{mm} / \mathrm{yr} \text {-simple } \\
\text { average }\end{array}$ & $\begin{array}{c}\text { average after } \\
1996.131\end{array}$ \\
\hline 0.0 & 1.3 & 0.000 & 1992.262 & 5-Apr-92 & & \\
\hline 0.8 & 0.9 & 0.170 & 1992.432 & 6-Jun-92 & & \\
\hline-5.0 & 1.0 & 0.514 & 1992.776 & $10-$ Oct-92 & & \\
\hline-4.6 & 2.1 & 0.661 & 1992.923 & 3-Dec-92 & & \\
\hline-0.9 & 2.3 & 0.861 & 1993.123 & 14-Feb-93 & & \\
\hline 0.1 & 1.9 & 1.034 & 1993.296 & 18-Apr-93 & 0.1 & \\
\hline 0.9 & 0.8 & 1.108 & 1993.370 & 15-May-93 & 0.8 & \\
\hline-3.2 & 0.9 & 1.376 & 1993.638 & 21-Aug-93 & -2.3 & \\
\hline-3.6 & 1.5 & 1.606 & 1993.868 & 13-Nov-93 & -2.2 & \\
\hline-1.0 & 1.6 & 1.798 & 1994.060 & 22-Jan-94 & -0.6 & \\
\hline 0.2 & 1.0 & 1.990 & 1994.252 & 2-Apr-94 & 0.1 & \\
\hline 0.3 & 1.7 & 2.204 & 1994.466 & 19-Jun-94 & 0.1 & \\
\hline-1.7 & 1.8 & 2.330 & 1994.592 & 4-Aug-94 & -0.7 & \\
\hline 1.1 & 0.8 & 2.508 & 1994.770 & 8-Oct-94 & 0.4 & \\
\hline 3.3 & 3.2 & 2.700 & 1994.962 & 17-Dec-94 & 1.2 & \\
\hline 1.8 & 1.5 & 2.891 & 1995.153 & 25-Feb-95 & 0.6 & \\
\hline 7.3 & 1.8 & 3.064 & 1995.326 & 29-Apr-95 & 2.4 & \\
\hline 4.4 & 0.7 & 3.220 & 1995.482 & 25-Jun-95 & 1.4 & \\
\hline 4.2 & 0.8 & 3.390 & 1995.652 & 26-Aug-95 & 1.2 & \\
\hline 4.6 & 2.9 & 3.546 & 1995.808 & $22-0 c t-95$ & 1.3 & \\
\hline 6.8 & 0.8 & 3.697 & 1995.959 & 16-Dec-95 & 1.8 & \\
\hline 23.4 & 3.4 & 3.869 & 1996.131 & 17-Feb-96 & 6.0 & \\
\hline 26.4 & 3.8 & 3.888 & 1996.150 & 24-Feb-96 & 6.8 & \\
\hline 23.6 & 3.8 & 3.929 & 1996.191 & 10-Mar-96 & 6.0 & \\
\hline 23.0 & 1.0 & 4.099 & 1996.361 & 11-May-96 & 5.6 & \\
\hline 25.7 & 4.2 & 4.271 & 1996.533 & 13-Jul-96 & 6.0 & \\
\hline 21.5 & 1.9 & 4.380 & 1996.642 & 22-Aug-96 & 4.9 & \\
\hline 23.0 & 1.7 & 4.539 & 1996.801 & $19-0 c t-96$ & 5.1 & \\
\hline 25.6 & 1.1 & 4.692 & 1996.954 & 14-Dec-96 & 5.5 & \\
\hline 25.8 & 2.3 & 4.845 & 1997.107 & 8-Feb-97 & 5.3 & 2.5 \\
\hline 25.2 & 2.0 & 5.017 & 1997.279 & 12-Apr-97 & 5.0 & 1.6 \\
\hline 24.9 & 1.3 & 5.193 & 1997.455 & 15-Jun-97 & 4.8 & 1.1 \\
\hline 24.0 & 1.3 & 5.385 & 1997.647 & 24-Aug-97 & 4.5 & 0.4 \\
\hline 25.7 & 1.0 & 5.535 & 1997.797 & $18-0 c t-97$ & 4.6 & 1.4 \\
\hline 27.0 & 1.8 & 5.727 & 1997.989 & 27-Dec-97 & 4.7 & 1.9 \\
\hline 27.8 & 0.4 & 5.900 & 1998.162 & 28-Feb-98 & 4.7 & 2.2 \\
\hline 26.5 & 1.4 & 6.053 & 1998.315 & 25-Apr-98 & 4.4 & 1.4 \\
\hline 28.3 & 1.1 & 6.226 & 1998.488 & 27-Jun-98 & 4.5 & 2.1 \\
\hline 26.7 & 1.2 & 6.423 & 1998.685 & 7-Sep-98 & 4.2 & 1.3 \\
\hline 27.8 & 1.0 & 6.628 & 1998.890 & 21-Nov-98 & 4.2 & 1.6 \\
\hline 32.2 & 0.3 & 6.820 & 1999.082 & 30-Jan-99 & 4.7 & 3.0 \\
\hline 32.8 & 1.1 & 6.957 & 1999.219 & 21-Mar-99 & 4.7 & 3.0 \\
\hline 33.3 & 1.2 & 7.127 & 1999.389 & 22-May-99 & 4.7 & 3.0 \\
\hline 31.1 & 3.8 & 7.300 & 1999.562 & 24-Jul-99 & 4.3 & 2.2 \\
\hline
\end{tabular}




$\begin{array}{llllcll}30.6 & 1.8 & 7.434 & 1999.696 & 11-\text { Sep-99 } & 4.1 & 2.0 \\ 32.8 & 3.8 & 7.587 & 1999.849 & 6 \text {-Nov-99 } & 4.3 & 2.5 \\ 35.1 & 3.7 & 7.817 & 2000.079 & \text { 29-Jan-00 } & 4.5 & 3.0 \\ 34.3 & 1.8 & 7.970 & 2000.232 & 25 \text {-Mar-00 } & 4.3 & 2.7 \\ 38.3 & 2.3 & 8.126 & 2000.388 & 21 \text {-May-00 } & 4.7 & 3.5 \\ 39.3 & 1.9 & 8.315 & 2000.577 & 29-J u l-00 & 4.7 & 3.6 \\ 42.8 & 3.0 & 8.544 & 2000.806 & 21-\text { Oct-00 } & 5.0 & 4.1 \\ 45.2 & 4.5 & 8.678 & 2000.940 & \text { 9-Dec-00 } & 5.2 & 4.5 \\ 42.9 & 3.3 & 8.850 & 2001.112 & 10-\text {-Eeb-01 } & 4.8 & 3.9\end{array}$


SF-28 Encina Way in Oakland Hayward Fault 33.39 km

\begin{tabular}{|c|c|c|c|c|c|}
\hline $\begin{array}{l}\text { movement } \\
(\mathrm{mm})\end{array}$ & std. dev. & \# of years & year & date & $\begin{array}{c}\mathrm{mm} / \mathrm{yr} \text {-simple } \\
\text { average }\end{array}$ \\
\hline 0.0 & 0.6 & 0.000 & 1989.693 & 10-Sep-89 & \\
\hline 10.7 & 1.1 & 2.927 & 1992.620 & 14-Aug-92 & 3.7 \\
\hline 19.5 & 0.5 & 3.365 & 1993.058 & 21-Jan-93 & 5.8 \\
\hline 18.2 & 3.2 & 3.581 & 1993.274 & 10-Apr-93 & 5.1 \\
\hline 25.0 & 1.9 & 3.948 & 1993.641 & 22-Aug-93 & 6.3 \\
\hline 24.9 & 0.9 & 4.099 & 1993.792 & 16-Oct-93 & 6.1 \\
\hline 25.9 & 1.3 & 4.332 & 1994.025 & 9-Jan-94 & 6.0 \\
\hline 25.4 & 2.7 & 4.523 & 1994.216 & 20-Mar-94 & 5.6 \\
\hline 28.5 & 1.2 & 4.734 & 1994.427 & 5-Jun-94 & 6.0 \\
\hline 27.4 & 1.1 & 4.888 & 1994.581 & 31-Jul-94 & 5.6 \\
\hline 27.0 & 0.5 & 5.096 & 1994.789 & 15-Oct-94 & 5.3 \\
\hline 28.0 & 2.3 & 5.271 & 1994.964 & 18-Dec-94 & 5.3 \\
\hline 28.2 & 0.7 & 5.463 & 1995.156 & 26-Feb-95 & 5.2 \\
\hline 29.2 & 0.4 & 5.671 & 1995.364 & 13-May-95 & 5.1 \\
\hline 27.7 & 1.7 & 5.847 & 1995.540 & 16-Jul-95 & 4.7 \\
\hline 30.6 & 0.6 & 5.948 & 1995.641 & 22-Aug-95 & 5.1 \\
\hline 24.7 & 0.8 & 6.192 & 1995.885 & 19-Nov-95 & 4.0 \\
\hline 30.6 & 1.0 & 6.285 & 1995.978 & 23-Dec-95 & 4.9 \\
\hline 32.0 & 0.7 & 6.572 & 1996.265 & 6-Apr-96 & 4.9 \\
\hline 31.1 & 3.0 & 6.802 & 1996.495 & 29-Jun-96 & 4.6 \\
\hline 32.1 & 1.5 & 6.944 & 1996.637 & 20-Aug-96 & 4.6 \\
\hline 31.1 & 2.2 & 7.110 & 1996.803 & 20-Oct-96 & 4.4 \\
\hline 31.5 & 1.3 & 7.244 & 1996.937 & 8-Dec-96 & 4.3 \\
\hline 32.0 & 0.3 & 7.397 & 1997.090 & 2-Feb-97 & 4.3 \\
\hline 33.4 & 1.5 & 7.608 & 1997.301 & 20-Apr-97 & 4.4 \\
\hline 31.0 & 0.6 & 7.743 & 1997.436 & 8-Jun-97 & 4.0 \\
\hline 30.6 & 0.8 & 7.937 & 1997.630 & 18-Aug-97 & 3.9 \\
\hline 27.5 & 2.8 & 8.143 & 1997.836 & 1-Nov-97 & 3.4 \\
\hline 33.0 & 0.6 & 8.433 & 1998.126 & 15-Feb-98 & 3.9 \\
\hline 35.7 & 0.7 & 8.567 & 1998.260 & 5-Apr-98 & 4.2 \\
\hline 35.7 & 0.7 & 8.721 & 1998.414 & 31-May-98 & 4.1 \\
\hline 33.4 & 0.7 & 8.986 & 1998.679 & 5-Sep-98 & 3.7 \\
\hline 39.2 & 0.8 & 9.162 & 1998.855 & 8-Nov-98 & 4.3 \\
\hline 39.3 & 0.6 & 9.370 & 1999.063 & 23-Jan-99 & 4.2 \\
\hline 41.9 & 0.5 & 9.507 & 1999.200 & 14-Mar-99 & 4.4 \\
\hline 41.6 & 1.0 & 9.775 & 1999.468 & 20-Jun-99 & 4.3 \\
\hline 39.6 & 1.7 & 9.986 & 1999.679 & 5-Sep-99 & 4.0 \\
\hline 36.3 & 0.5 & 10.195 & 1999.888 & 20-Nov-99 & 3.6 \\
\hline 39.9 & 1.9 & 10.408 & 2000.101 & 6-Feb-00 & 3.8 \\
\hline 41.6 & 1.5 & 10.616 & 2000.309 & 22-Apr-00 & 3.9 \\
\hline 41.7 & 1.2 & 10.829 & 2000.522 & 9-Jul-00 & 3.9 \\
\hline 41.7 & 1.3 & 11.017 & 2000.710 & 16-Sep-00 & 3.8 \\
\hline 41.8 & 0.8 & 11.173 & 2000.866 & 12-Nov-00 & 3.7 \\
\hline 43.0 & 0.8 & 11.362 & 2001.055 & 20-Jan-01 & 3.8 \\
\hline
\end{tabular}


SF-29 LaSalle Ave in Oakland Hayward Fault 23.92 km

\begin{tabular}{|c|c|c|c|c|c|}
\hline $\begin{array}{l}\text { movement } \\
(\mathrm{mm})\end{array}$ & std. dev. & \# of years & year & date & $\begin{array}{c}\mathrm{mm} / \mathrm{yr} \text {-simple } \\
\text { average }\end{array}$ \\
\hline 0.0 & 1.5 & 0.000 & 1993.112 & 10-Feb-93 & \\
\hline 3.8 & 1.3 & 0.184 & 1993.296 & 18-Apr-93 & \\
\hline 3.1 & 1.3 & 0.529 & 1993.641 & 22-Aug-93 & \\
\hline 1.3 & 1.2 & 0.680 & 1993.792 & 16-Oct-93 & \\
\hline 5.8 & 1.8 & 0.913 & 1994.025 & 9-Jan-94 & \\
\hline 5.8 & 2.6 & 1.104 & 1994.216 & 20-Mar-94 & 5.3 \\
\hline 6.1 & 3.4 & 1.315 & 1994.427 & 5-Jun-94 & 4.6 \\
\hline 8.6 & 1.7 & 1.469 & 1994.581 & 31-Jul-94 & 5.9 \\
\hline 9.2 & 1.6 & 1.677 & 1994.789 & $15-O c t-94$ & 5.5 \\
\hline 9.9 & 1.3 & 1.852 & 1994.964 & 18-Dec-94 & 5.3 \\
\hline 7.8 & 2.1 & 2.044 & 1995.156 & 26-Feb-95 & 3.8 \\
\hline 9.9 & 1.0 & 2.252 & 1995.364 & 13-May-95 & 4.4 \\
\hline 10.6 & 1.8 & 2.428 & 1995.540 & 16-Jul-95 & 4.4 \\
\hline 11.2 & 0.6 & 2.524 & 1995.636 & 20-Aug-95 & 4.4 \\
\hline 10.4 & 9.0 & 2.773 & 1995.885 & 19-Nov-95 & 3.8 \\
\hline 12.1 & 1.6 & 2.828 & 1995.940 & 9-Dec-95 & 4.3 \\
\hline 12.3 & 1.9 & 3.000 & 1996.112 & 10-Feb-96 & 4.1 \\
\hline 14.2 & 0.8 & 3.194 & 1996.306 & 21-Apr-96 & 4.4 \\
\hline 14.6 & 2.5 & 3.325 & 1996.437 & 8-Jun-96 & 4.4 \\
\hline 14.6 & 0.7 & 3.519 & 1996.631 & 18-Aug-96 & 4.2 \\
\hline 19.0 & 0.9 & 3.691 & 1996.803 & 20-Oct-96 & 5.1 \\
\hline 14.4 & 1.4 & 3.825 & 1996.937 & 8-Dec-96 & 3.8 \\
\hline 17.5 & 1.0 & 3.978 & 1997.090 & 2-Feb-97 & 4.4 \\
\hline 16.0 & 1.2 & 4.189 & 1997.301 & 20-Apr-97 & 3.8 \\
\hline 16.5 & 2.2 & 4.324 & 1997.436 & 8-Jun-97 & 3.8 \\
\hline 18.0 & 0.7 & 4.515 & 1997.627 & 17-Aug-97 & 4.0 \\
\hline 20.5 & 0.5 & 4.707 & 1997.819 & $26-0 c t-97$ & 4.4 \\
\hline 21.1 & 1.9 & 4.861 & 1997.973 & 21-Dec-97 & 4.3 \\
\hline 20.3 & 1.1 & 5.088 & 1998.200 & 14-Mar-98 & 4.0 \\
\hline 22.6 & 1.5 & 5.302 & 1998.414 & 31-May-98 & 4.3 \\
\hline 20.7 & 0.6 & 5.551 & 1998.663 & 30-Aug-98 & 3.7 \\
\hline 22.8 & 1.7 & 5.683 & 1998.795 & 17-Oct-98 & 4.0 \\
\hline 23.4 & 1.1 & 5.855 & 1998.967 & 19-Dec-98 & 4.0 \\
\hline 23.3 & 0.9 & 6.030 & 1999.142 & 21-Feb-99 & 3.9 \\
\hline 25.0 & 0.6 & 6.203 & 1999.315 & 25-Apr-99 & 4.0 \\
\hline 25.2 & 1.4 & 6.356 & 1999.468 & 20-Jun-99 & 4.0 \\
\hline 23.3 & 0.8 & 6.548 & 1999.660 & 29-Aug-99 & 3.6 \\
\hline 25.6 & 1.2 & 6.778 & 1999.890 & 21-Nov-99 & 3.8 \\
\hline 27.9 & 0.8 & 6.932 & 2000.044 & 16-Jan-00 & 4.0 \\
\hline 29.8 & 1.3 & 7.410 & 2000.522 & 9-Jul-00 & 4.0 \\
\hline 28.4 & 1.7 & 7.598 & 2000.710 & 16-Sep-00 & 3.7 \\
\hline 33.8 & 0.9 & 7.754 & 2000.866 & 12-Nov-00 & 4.4 \\
\hline 33.0 & 1.2 & 7.943 & 2001.055 & 20-Jan-01 & 4.2 \\
\hline
\end{tabular}


SF-30 Florida Ave in Berkeley Hayward Fault 14.05 km

\begin{tabular}{|c|c|c|c|c|c|c|}
\hline $\begin{array}{l}\text { movement } \\
\quad(\mathrm{mm})\end{array}$ & std. dev. & \# of years & year & date & $\begin{array}{c}\mathrm{mm} / \mathrm{yr} \text {-simple } \\
\text { average }\end{array}$ & $\begin{array}{c}\text { average after } \\
1997.625\end{array}$ \\
\hline 0.0 & 0.3 & 0.000 & 1992.615 & 12-Aug-92 & & \\
\hline-1.4 & 1.2 & 0.497 & 1993.112 & 10-Feb-93 & & \\
\hline-1.9 & 1.5 & 0.689 & 1993.304 & 21-Apr-93 & & \\
\hline 0.1 & 1.0 & 0.966 & 1993.581 & 31-Jul-93 & 0.1 & \\
\hline-1.5 & 1.6 & 1.138 & 1993.753 & 2-Oct-93 & -1.3 & \\
\hline-1.1 & 0.6 & 1.330 & 1993.945 & 11-Dec-93 & -0.8 & \\
\hline-0.8 & 0.7 & 1.506 & 1994.121 & 13-Feb-94 & -0.5 & \\
\hline 0.2 & 0.4 & 1.659 & 1994.274 & 10-Apr-94 & 0.1 & \\
\hline-1.1 & 0.5 & 1.829 & 1994.444 & 11-Jun-94 & -0.6 & \\
\hline-1.0 & 0.3 & 1.966 & 1994.581 & 31-Jul-94 & -0.5 & \\
\hline-1.6 & 0.6 & 2.174 & 1994.789 & $15-$ Oct-94 & -0.7 & \\
\hline 0.9 & 0.4 & 2.349 & 1994.964 & 18-Dec-94 & 0.4 & \\
\hline 1.0 & 0.6 & 2.541 & 1995.156 & 26-Feb-95 & 0.4 & \\
\hline 1.2 & 0.7 & 2.749 & 1995.364 & 13-Мay-95 & 0.4 & \\
\hline-0.1 & 0.7 & 2.925 & 1995.540 & 16-Jul-95 & 0.0 & \\
\hline 0.8 & 0.2 & 3.018 & 1995.633 & 19-Aug-95 & 0.3 & \\
\hline-0.5 & 0.5 & 3.270 & 1995.885 & 19-Nov-95 & -0.2 & \\
\hline 0.3 & 0.4 & 3.325 & 1995.940 & 9-Dec-95 & 0.1 & \\
\hline 0.7 & 2.0 & 3.497 & 1996.112 & 10-Feb-96 & 0.2 & \\
\hline 1.7 & 1.3 & 3.691 & 1996.306 & 21-Apr-96 & 0.5 & \\
\hline 1.9 & 1.6 & 3.822 & 1996.437 & 8-Jun-96 & 0.5 & \\
\hline 0.0 & 0.8 & 4.013 & 1996.628 & 17-Aug-96 & 0.0 & \\
\hline 1.4 & 0.5 & 4.188 & 1996.803 & 20-Oct-96 & 0.3 & \\
\hline 1.5 & 0.5 & 4.322 & 1996.937 & 8-Dec-96 & 0.3 & \\
\hline 2.2 & 0.3 & 4.475 & 1997.090 & 2-Feb-97 & 0.5 & \\
\hline 3.1 & 0.8 & 4.706 & 1997.321 & 27-Apr-97 & 0.7 & \\
\hline 3.1 & 0.9 & 5.010 & 1997.625 & 16-Aug-97 & 0.6 & \\
\hline 5.4 & 1.0 & 6.045 & 1998.660 & 29-Aug-98 & 0.9 & 2.2 \\
\hline 8.0 & 1.0 & 7.062 & 1999.677 & 4-Sep-99 & 1.1 & 2.4 \\
\hline 12.1 & 2.8 & 8.251 & 2000.866 & 12-Nov-00 & 1.5 & 2.8 \\
\hline
\end{tabular}


SF-31 Sanford Ranch Road near Ukiah Maacama Fault

\begin{tabular}{|c|c|c|c|c|c|}
\hline $\begin{array}{l}\text { movement } \\
\quad(\mathrm{mm})\end{array}$ & std. dev. & \# of years & year & date & $\begin{array}{c}\mathrm{mm} / \mathrm{yr} \text {-simple } \\
\text { average }\end{array}$ \\
\hline 0.0 & 1.8 & 0.000 & 1993.389 & 22-May-93 & \\
\hline-1.3 & 1.3 & 0.230 & 1993.619 & 14-Aug-93 & \\
\hline 1.1 & 1.4 & 0.422 & 1993.811 & 23-Oct-93 & \\
\hline 2.3 & 3.6 & 0.825 & 1994.214 & 19-Mar-94 & \\
\hline 1.9 & 1.4 & 0.962 & 1994.351 & 8-May-94 & 2.0 \\
\hline 8.4 & 3.4 & 1.132 & 1994.521 & 9-Jul-94 & 7.4 \\
\hline 8.0 & 7.4 & 1.304 & 1994.693 & 10-Sep-94 & 6.1 \\
\hline 8.8 & 3.1 & 1.496 & 1994.885 & 19-Nov-94 & 5.9 \\
\hline 12.2 & 2.1 & 1.669 & 1995.058 & 21-Jan-95 & 7.3 \\
\hline 11.5 & 2.0 & 1.841 & 1995.230 & 25-Mar-95 & 6.2 \\
\hline 14.4 & 2.1 & 2.052 & 1995.441 & 10-Jun-95 & 7.0 \\
\hline 8.4 & 0.9 & 2.225 & 1995.614 & 12-Aug-95 & 3.8 \\
\hline 8.8 & 2.8 & 2.438 & 1995.827 & 29-Oct-95 & 3.6 \\
\hline 13.4 & 1.2 & 2.666 & 1996.055 & 20-Jan-96 & 5.0 \\
\hline 9.3 & 9.6 & 2.857 & 1996.246 & 30-Mar-96 & 3.3 \\
\hline 9.4 & 4.0 & 3.067 & 1996.456 & 15-Jun-96 & 3.1 \\
\hline 10.1 & 1.5 & 3.278 & 1996.667 & 31-Aug-96 & 3.1 \\
\hline 13.5 & 2.2 & 3.453 & 1996.842 & 3-Nov-96 & 3.9 \\
\hline 16.7 & 1.0 & 3.699 & 1997.088 & 1-Feb-97 & 4.5 \\
\hline 13.0 & 2.7 & 3.871 & 1997.260 & 5-Apr-97 & 3.4 \\
\hline 13.8 & 2.4 & 4.063 & 1997.452 & 14-Jun-97 & 3.4 \\
\hline 17.7 & 4.6 & 4.216 & 1997.605 & 9-Aug-97 & 4.2 \\
\hline 18.0 & 0.9 & 4.427 & 1997.816 & $25-$ Oct-97 & 4.1 \\
\hline 20.5 & 3.2 & 4.581 & 1997.970 & 20-Dec-97 & 4.5 \\
\hline 24.0 & 4.0 & 4.792 & 1998.181 & 7-Mar-98 & 5.0 \\
\hline 23.4 & 2.9 & 4.964 & 1998.353 & 9-May-98 & 4.7 \\
\hline 22.3 & 2.6 & 5.137 & 1998.526 & 11-Jul-98 & 4.3 \\
\hline 20.5 & 3.4 & 5.329 & 1998.718 & 19-Sep-98 & 3.8 \\
\hline 25.3 & 2.1 & 5.504 & 1998.893 & 22-Nov-98 & 4.6 \\
\hline 23.0 & 2.2 & 5.677 & 1999.066 & 24-Jan-99 & 4.1 \\
\hline 25.4 & 2.3 & 5.827 & 1999.216 & 20-Mar-99 & 4.4 \\
\hline 23.5 & 2.3 & 5.981 & 1999.370 & 15-May-99 & 3.9 \\
\hline 25.1 & 3.5 & 6.175 & 1999.564 & 25-Jul-99 & 4.1 \\
\hline 24.6 & 2.9 & 6.386 & 1999.775 & 10-Oct-99 & 3.9 \\
\hline 25.3 & 5.2 & 6.556 & 1999.945 & 11-Dec-99 & 3.9 \\
\hline 26.1 & 3.8 & 6.805 & 2000.194 & 11-Mar-00 & 3.8 \\
\hline 34.2 & 3.7 & 6.977 & 2000.366 & 13-May-00 & 4.9 \\
\hline 33.4 & 1.6 & 7.149 & 2000.538 & 15-Jul-00 & 4.7 \\
\hline 38.4 & 1.8 & 7.362 & 2000.751 & $1-0 c t-00$ & 5.2 \\
\hline 38.7 & 4.8 & 7.532 & 2000.921 & 2-Dec-00 & 5.1 \\
\hline 35.0 & 4.3 & 7.762 & 2001.151 & 24-Feb-01 & 4.5 \\
\hline
\end{tabular}


SF-32 Welch Creek Road in Sunol Valley Calaveras Fault

$\begin{array}{cccccc}\begin{array}{c}\text { movement } \\ (\mathrm{mm})\end{array} & \text { std. dev. } & \text { \# of years } & \begin{array}{c}\text { year } \\ \text { date }\end{array} & \begin{array}{c}\mathrm{mm} \text { /yr-simple } \\ \text { average }\end{array} \\ 0.0 & 0.8 & 0.000 & 1997.066 & \text { 24-Jan-97 } & \\ -1.0 & 1.9 & 0.159 & 1997.225 & \text { 23-Mar-97 } & \\ 4.1 & 1.6 & 0.348 & 1997.414 & \text { 31-May-97 } & \\ 6.8 & 0.5 & 0.523 & 1997.589 & \text { 3-Aug-97 } & \\ 3.4 & 1.4 & 0.696 & 1997.762 & \text { 5-Oct-97 } & \\ 3.4 & 1.7 & 0.866 & 1997.932 & \text { 6-Dec-97 } & 3.9 \\ -1.4 & 1.3 & 1.156 & 1998.222 & \text { 22-Mar-98 } & -1.2 \\ 2.7 & 2.7 & 1.419 & 1998.485 & \text { 26-Jun-98 } & 1.9 \\ 6.6 & 1.1 & 1.671 & 1998.737 & \text { 26-Sep-98 } & 3.9 \\ 8.9 & 1.3 & 1.846 & 1998.912 & \text { 29-Nov-98 } & 4.8 \\ 5.0 & 0.7 & 2.074 & 1999.140 & \text { 20-Feb-99 } & 2.4 \\ 7.5 & 0.7 & 2.227 & 1999.293 & \text { 17-Apr-99 } & 3.4 \\ 8.9 & 0.8 & 2.400 & 1999.466 & \text { 19-Jun-99 } & 3.7 \\ 13.5 & 0.7 & 2.745 & 1999.811 & \text { 23-Oct-99 } & 4.9 \\ 12.7 & 2.8 & 2.898 & 1999.964 & \text { 18-Dec-99 } & 4.4 \\ 10.4 & 1.0 & 3.090 & 2000.156 & \text { 26-Feb-00 } & 3.4 \\ 7.4 & 2.1 & 3.262 & 2000.328 & \text { 29-Apr-00 } & 2.3 \\ 14.9 & 0.3 & 3.453 & 2000.519 & \text { 8-Jul-00 } & 4.3 \\ 14.8 & 1.9 & 3.666 & 2000.732 & \text { 24-Sep-00 } & 4.0 \\ 14.1 & 1.3 & 3.836 & 2000.902 & \text { 25-Nov-00 } & 3.7 \\ 13.9 & 1.1 & 4.008 & 2001.074 & \text { 27-Jan-01 } & 3.5\end{array}$


SF-33 Coyote Ranch near Coyote Lake Calaveras Fault

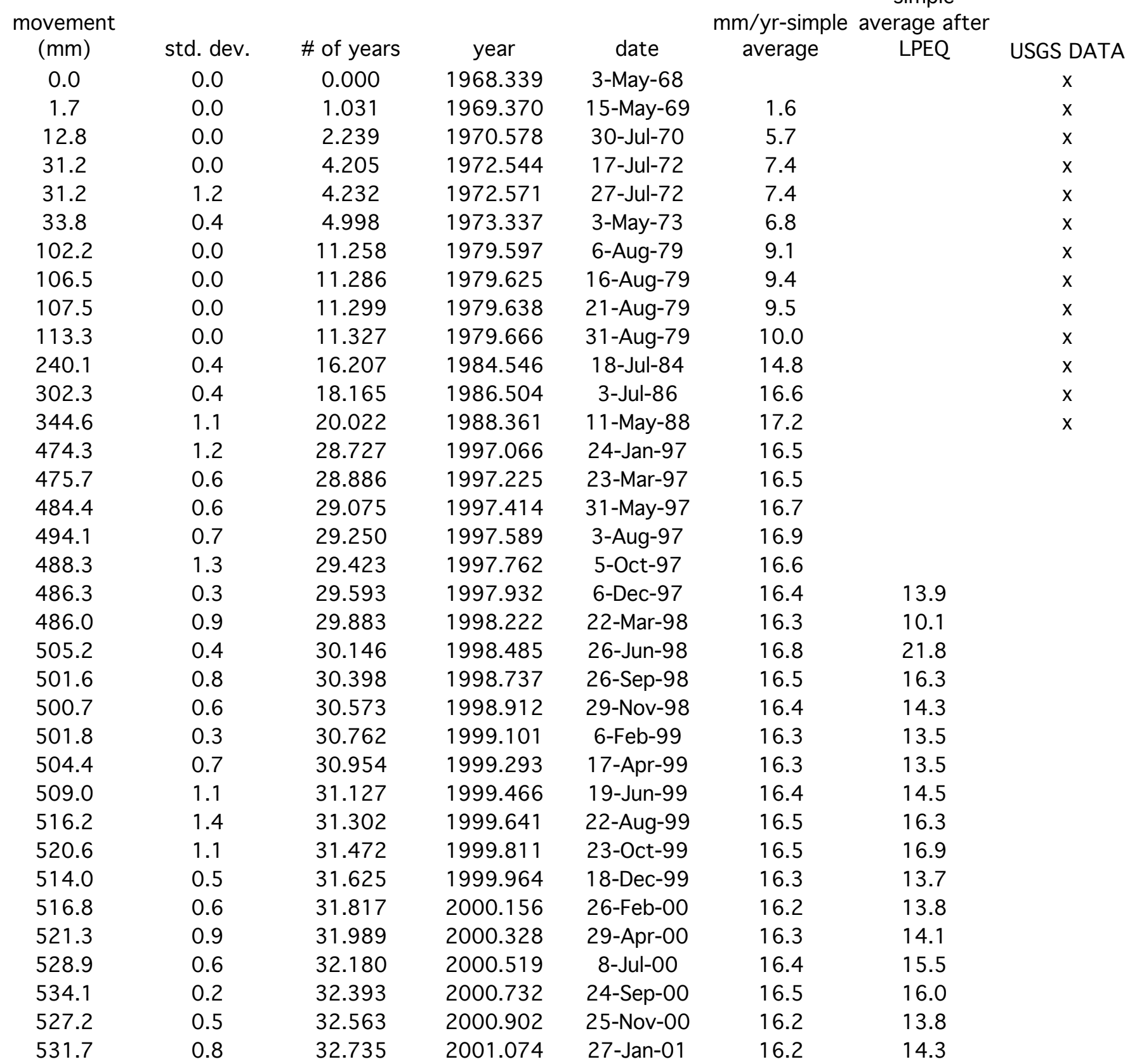




\section{SF-34 Thors Bay Road in El Cerrito Hayward Fault 10.83 km}

\begin{tabular}{|c|c|c|c|c|c|}
\hline $\begin{array}{l}\text { movement } \\
\quad(\mathrm{mm})\end{array}$ & std. dev. & \# of years & year & date & $\begin{array}{c}\mathrm{mm} / \mathrm{yr} \text {-simple } \\
\text { average }\end{array}$ \\
\hline 0.0 & 1.0 & 0.000 & 1989.748 & 30-Sep-89 & \\
\hline 14.5 & 1.0 & 2.867 & 1992.615 & 12-Aug-92 & 5.1 \\
\hline 21.3 & 1.0 & 3.304 & 1993.052 & 19-Jan-93 & 6.4 \\
\hline 23.7 & 1.0 & 4.830 & 1994.578 & 30-Jul-94 & 4.9 \\
\hline 29.0 & 0.4 & 5.885 & 1995.633 & 19-Aug-95 & 4.9 \\
\hline 32.9 & 0.9 & 6.880 & 1996.628 & 17-Aug-96 & 4.8 \\
\hline 35.7 & 2.1 & 7.688 & 1997.436 & 8-Jun-97 & 4.6 \\
\hline 37.3 & 0.5 & 7.877 & 1997.625 & 16-Aug-97 & 4.7 \\
\hline 37.6 & 1.1 & 8.071 & 1997.819 & 26-Oct-97 & 4.7 \\
\hline 40.4 & 1.1 & 8.225 & 1997.973 & 21-Dec-97 & 4.9 \\
\hline 40.2 & 0.7 & 8.378 & 1998.126 & 15-Feb-98 & 4.8 \\
\hline 40.4 & 0.7 & 8.512 & 1998.260 & 5-Apr-98 & 4.7 \\
\hline 42.2 & 0.3 & 8.704 & 1998.452 & 14-Jun-98 & 4.8 \\
\hline 41.0 & 0.4 & 8.912 & 1998.660 & 29-Aug-98 & 4.6 \\
\hline 39.7 & 0.6 & 9.047 & 1998.795 & 17-Oct-98 & 4.4 \\
\hline 45.4 & 0.4 & 9.181 & 1998.929 & 5-Dec-98 & 4.9 \\
\hline 45.5 & 1.0 & 9.394 & 1999.142 & 21-Feb-99 & 4.8 \\
\hline 45.8 & 1.5 & 9.567 & 1999.315 & 25-Apr-99 & 4.8 \\
\hline 45.3 & 0.1 & 9.720 & 1999.468 & 20-Jun-99 & 4.7 \\
\hline 45.9 & 0.6 & 9.929 & 1999.677 & 4-Sep-99 & 4.6 \\
\hline 44.5 & 0.4 & 10.120 & 1999.868 & 13-Nov-99 & 4.4 \\
\hline 47.2 & 1.6 & 10.296 & 2000.044 & 16-Jan-00 & 4.6 \\
\hline 48.0 & 0.5 & 10.487 & 2000.235 & 26-Mar-00 & 4.6 \\
\hline 49.6 & 1.1 & 10.656 & 2000.404 & 27-May-00 & 4.7 \\
\hline 45.6 & 0.6 & 10.831 & 2000.579 & 30-Jul-00 & 4.2 \\
\hline 48.9 & 0.8 & 11.080 & 2000.828 & 29-Oct-00 & 4.4 \\
\hline 51.3 & 2.0 & 11.310 & 2001.058 & 21-Jan-01 & 4.5 \\
\hline
\end{tabular}


Hayward Fault USGS-SFSU Afterslip Site $08.37 \mathrm{~km}$-Olive Ave.

$\begin{array}{cccccc}\begin{array}{c}\text { movement } \\ (\mathrm{mm})\end{array} & \text { std. dev. } & \text { \# of years } & \begin{array}{c}\text { year } \\ \text { date }\end{array} & \begin{array}{c}\mathrm{mm} / \mathrm{yr} . \\ \text { simple } \\ \text { average }\end{array} \\ 0.0 & 0.0 & 0.000 & 1989.748 & \text { 30-Sep-89 } & \\ 14.3 & 0.8 & 2.867 & 1992.615 & 12-\text { Aug-92 } & 5.0 \\ 26.9 & 0.9 & 4.830 & 1994.578 & 30-J u l-94 & 5.6 \\ 31.1 & 1.5 & 5.885 & 1995.633 & \text { 19-Aug-95 } & 5.3 \\ 34.1 & 0.6 & 6.880 & 1996.628 & \text { 17-Aug-96 } & 5.0 \\ 40.7 & 0.8 & 7.877 & 1997.625 & 16 \text {-Aug-97 } & 5.2 \\ 42.6 & 1.2 & 8.912 & 1998.660 & \text { 29-Aug-98 } & 4.8 \\ 50.3 & 0.6 & 9.929 & 1999.677 & \text { 4-Sep-99 } & 5.1 \\ 57.4 & 1.0 & 11.118 & 2000.866 & \text { 12-Nov-00 } & 5.2\end{array}$


Hayward Fault USGS-SFSU Afterslip Site

$17.82 \mathrm{~km}$-Cal Stadium

$\begin{array}{cccccc}\begin{array}{c}\text { movement } \\ (\mathrm{mm})\end{array} & \text { std. dev. } & \text { \# of years } & \text { year } & \text { date } & \begin{array}{c}\text { simple } \\ \text { average }\end{array} \\ 0.0 & & 0.000 & 1966.912 & \text { 29-Nov-66 } & \\ 101.5 & & 21.503 & 1988.415 & 31 \text {-May-88 } & 4.7 \\ 120.4 & & 25.755 & 1992.667 & 31 \text {-Aug-92 } & 4.7 \\ 124.0 & 1.4 & 26.576 & 1993.488 & 27-J u n-93 & 4.7 \\ 141.1 & 0.6 & 30.521 & 1997.433 & 7-J u n-97 & 4.6 \\ 148.4 & 1.0 & 31.748 & 1998.660 & \text { 29-Aug-98 } & 4.7 \\ 153.8 & 0.4 & 32.746 & 1999.658 & \text { 28-Aug-99 } & 4.7 \\ 158.9 & 1.1 & 33.916 & 2000.828 & \text { 29-Oct-00 } & 4.7\end{array}$


Hayward Fault USGS-SFSU Afterslip Site 20.84 km-Temescal

$\begin{array}{cccccc}\begin{array}{c}\text { movement } \\ (\mathrm{mm})\end{array} & \text { std. dev. } & \begin{array}{c}\text { \# of years } \\ 0.000\end{array} & \begin{array}{c}\text { year } \\ 1974.258\end{array} & \begin{array}{c}\text { date } \\ \text { 4-Apr-74 }\end{array} & \begin{array}{c}\text { simple } \\ \text { average }\end{array} \\ 0.0 & & 19.860 & 1994.118 & 12 \text {-Feb-94 } & 3.8 \\ 74.8 & 1.2 & 20.326 & 1994.584 & 1 \text {-Aug-94 } & 3.7 \\ 74.9 & 0.3 & 21.383 & 1995.641 & 22-\text { Aug-95 } & 3.8 \\ 80.3 & 0.4 & 24.482 & 1998.740 & 27-\text { Sep-98 } & 3.8 \\ 92.1 & 1.1 & 25.438 & 1999.696 & 11-\text { Sep-99 } & 3.8 \\ 97.5 & 1.4 & 26.608 & 2000.866 & 12-\text { Nov-00 } & 3.9 \\ 102.8 & 0.8 & & & & \end{array}$


Hayward Fault USGS-SFSU Afterslip Site 25.98 km-Lincoln Ave.

$\begin{array}{ccccccc}\begin{array}{c}\text { movement } \\ (\mathrm{mm})\end{array} & \text { std. dev. } & \begin{array}{c}\text { \# of years } \\ 0.000\end{array} & \begin{array}{c}\text { year } \\ 1970.290\end{array} & \begin{array}{c}\text { date } \\ \text { 16-Apr-70 }\end{array} & \begin{array}{c}\text { simple } \\ \text { average }\end{array} & \begin{array}{c}\text { after } \\ 1988.358\end{array} \\ 0.0 & & 18.068 & 1988.358 & 10 \text {-May-88 } & 4.0 & \\ 72.0 & & 22.330 & 1992.620 & 14-\text { Aug-92 } & 3.7 & 2.6 \\ 82.9 & & 24.294 & 1994.584 & \text { 1-Aug-94 } & 3.7 & 3.0 \\ 90.7 & 1.9 & 25.351 & 1995.641 & 22 \text {-Aug-95 } & 3.7 & 3.0 \\ 93.6 & 2.5 & 26.347 & 1996.637 & 20 \text {-Aug-96 } & 3.7 & 3.2 \\ 98.5 & 1.4 & 27.340 & 1997.630 & 18 \text {-Aug-97 } & 3.7 & 3.1 \\ 101.2 & 0.5 & 28.395 & 1998.685 & \text { 7-Sep-98 } & 3.6 & 3.1 \\ 103.5 & 3.1 & 29.406 & 1999.696 & 11 \text {-Sep-99 } & 3.6 & 3.1 \\ 107.1 & 0.7 & 30.576 & 2000.866 & \text { 12-Nov-00 } & 3.6 & 3.1 \\ 110.5 & 0.7 & & & & & \end{array}$


Hayward Fault USGS-SFSU Afterslip Site $27.81 \mathrm{~km}-39$ th Ave.

\begin{tabular}{|c|c|c|c|c|c|c|}
\hline $\begin{array}{l}\text { movement } \\
\quad(\mathrm{mm})\end{array}$ & std. dev. & \# of years & $\begin{array}{c}\text { year } \\
1974.274\end{array}$ & $\begin{array}{c}\text { date } \\
\text { 10-Apr-74 }\end{array}$ & $\begin{array}{c}\text { simple } \\
\text { average }\end{array}$ & $\begin{array}{c}\text { after } \\
1988.358\end{array}$ \\
\hline 201.0 & & 14.084 & 1988.358 & 10-May-88 & 14.3 & \\
\hline 207.6 & 0.9 & 18.343 & 1992.617 & 13-Aug-92 & 11.3 & 1.5 \\
\hline 224.2 & 2.4 & 19.701 & 1993.975 & 22-Dec-93 & 11.4 & 4.1 \\
\hline 217.1 & 3.2 & 20.310 & 1994.584 & 1-Aug-94 & 10.7 & 2.6 \\
\hline 227.0 & 0.6 & 21.362 & 1995.636 & 20-Aug-95 & 10.6 & 3.6 \\
\hline 229.0 & 1.9 & 22.357 & 1996.631 & 18-Aug-96 & 10.2 & 3.4 \\
\hline 233.6 & 2.9 & 23.353 & 1997.627 & 17-Aug-97 & 10.0 & 3.5 \\
\hline 238.9 & 1.1 & 24.389 & 1998.663 & 30-Aug-98 & 9.8 & 3.7 \\
\hline 242.4 & 0.3 & 25.386 & 1999.660 & 29-Aug-99 & 9.5 & 3.7 \\
\hline 247.4 & 2.1 & 26.589 & 2000.863 & 11-Nov-00 & 9.3 & 3.7 \\
\hline
\end{tabular}


Hayward Fault USGS-SFSU Afterslip Site 30.68 km-73rd Ave.

$\begin{array}{cccccc}\begin{array}{c}\text { movement } \\ (\mathrm{mm})\end{array} & \text { std. dev. } & \text { \# of years } & \text { year } & \text { date } & \begin{array}{c}\text { simple } \\ \text { average }\end{array} \\ 0.0 & 0.7 & 0.000 & 1993.389 & \text { 22-May-93 } & \\ 4.1 & 0.7 & 1.195 & 1994.584 & 1 \text {-Aug-94 } & 3.4 \\ 5.5 & 1.0 & 2.247 & 1995.636 & 20 \text {-Aug-95 } & 2.4 \\ 7.6 & 2.1 & 3.242 & 1996.631 & 18 \text {-Aug-96 } & 2.3 \\ 11.8 & 0.7 & 4.238 & 1997.627 & 17 \text {-Aug-97 } & 2.8 \\ 12.2 & 0.2 & 5.310 & 1998.699 & 12-\text { Sep-98 } & 2.3 \\ 17.5 & 1.0 & 6.271 & 1999.660 & 29 \text {-Aug-99 } & 2.8 \\ 21.3 & 0.3 & 7.436 & 2000.825 & 28 \text {-Oct-00 } & 2.9\end{array}$


Hayward Fault USGS-SFSU Afterslip Site 36.55 km-Chabot Park

$\begin{array}{cccccc}\begin{array}{c}\text { movement } \\ (\mathrm{mm})\end{array} & \text { std. dev. } & \text { \# of years } & \text { year } & \text { date } & \begin{array}{c}\text { simple } \\ \text { average }\end{array} \\ 0.0 & 2.0 & 0.000 & 1993.389 & \text { 22-May-93 } & \\ 5.2 & 1.0 & 1.197 & 1994.586 & \text { 02-Aug-94 } & 4.3 \\ 12.7 & 2.7 & 2.255 & 1995.644 & \text { 23-Aug-95 } & 5.6 \\ 18.3 & 0.7 & 3.245 & 1996.634 & \text { 19-Aug-96 } & 5.6 \\ 23.0 & 1.9 & 4.244 & 1997.633 & \text { 19-Aug-97 } & 5.4 \\ 28.7 & 1.0 & 5.290 & 1998.679 & \text { 05-Sep-98 } & 5.4 \\ 37.9 & 1.1 & 6.290 & 1999.679 & \text { 05-Sep-99 } & 6.0 \\ 37.3 & 1.4 & 7.436 & 2000.825 & \text { 28-Oct-00 } & 5.0\end{array}$


Hayward Fault USGS-SFSU Afterslip Site 38.28 km-Fairmont Dr.

$\begin{array}{cccccc}\begin{array}{c}\text { movement } \\ (\mathrm{mm})\end{array} & \text { std. dev. } & \text { \# of years } & \text { year } & \text { date } & \begin{array}{c}\text { simple } \\ \text { average }\end{array} \\ 0.0 & 1.2 & 0.000 & 1997.627 & 17-\text { Aug-97 } & \\ 2.0 & 0.8 & 1.036 & 1998.663 & 30 \text {-Aug-98 } & 1.9 \\ 7.6 & 2.0 & 2.033 & 1999.660 & 29 \text {-Aug-99 } & 3.7 \\ 12.7 & 2.0 & 3.198 & 2000.825 & 28 \text {-Oct-00 } & 4.0\end{array}$




\begin{tabular}{|c|c|c|c|c|c|}
\hline $\begin{array}{l}\text { movement } \\
(\mathrm{mm})\end{array}$ & std. dev. & \# of years & year & date & $\begin{array}{c}\mathrm{mm} / \mathrm{yr} \text {. } \\
\text { simple } \\
\text { average }\end{array}$ \\
\hline 0.0 & 1.3 & 0.000 & 1992.620 & 14-Aug-92 & \\
\hline 18.2 & 1.7 & 1.966 & 1994.586 & 2-Aug-94 & 9.3 \\
\hline 22.9 & 0.6 & 3.016 & 1995.636 & 20-Aug-95 & 7.6 \\
\hline 26.3 & 0.7 & 4.011 & 1996.631 & 18-Aug-96 & 6.6 \\
\hline 30.5 & 0.4 & 5.007 & 1997.627 & 17-Aug-97 & 6.1 \\
\hline 36.1 & 0.6 & 6.043 & 1998.663 & 30-Aug-98 & 6.0 \\
\hline 40.6 & 0.9 & 7.040 & 1999.660 & 29-Aug-99 & 5.8 \\
\hline 44.9 & 1.1 & 8.205 & 2000.825 & 28-Oct-00 & 5.5 \\
\hline
\end{tabular}


Hayward Fault USGS-SFSU Afterslip Site

45.64 km-Palisade Dr.

$\begin{array}{cccccc}\begin{array}{c}\text { movement } \\ (\mathrm{mm})\end{array} & \text { std. dev. } & \text { \# of years } & \text { year } & \begin{array}{c}\text { date } \\ \text { 27-Jan-77 }\end{array} & \text { average } \\ 0.0 & & 0.000 & 1977.074 & \\ 73.1 & & 15.735 & 1992.809 & 22-\text {-Oct-92 } & 4.6 \\ 78.5 & 2.5 & 20.556 & 1997.630 & 18 \text {-Aug-97 } & 3.8 \\ 86.2 & 3.0 & 21.589 & 1998.663 & \text { 30-Aug-98 } & 4.0 \\ 92.3 & 2.1 & 22.603 & 1999.677 & \text { 4-Sep-99 } & 4.1 \\ 100.0 & 2.1 & 23.732 & 2000.806 & 21 \text {-Oct-00 } & 4.2\end{array}$


Hayward Fault USGS-SFSU Afterslip Site

47.72 km-Holy Sepulchre Cemetery

$\begin{array}{cccccc}\begin{array}{c}\text { movement } \\ (\mathrm{mm})\end{array} & \text { std. dev. } & \text { \# of years } & \text { year } & \text { date } & \text { simple } \\ 0.0 & 1.0 & 0.000 & 1994.589 & \text { 3-Aug-94 } & \\ 9.2 & 1.2 & 1.052 & 1995.641 & \text { 22-Aug-95 } & 8.7 \\ 15.7 & 3.2 & 2.045 & 1996.634 & 19 \text {-Aug-96 } & 7.7 \\ 22.5 & 1.0 & 3.041 & 1997.630 & 18 \text {-Aug-97 } & 7.4 \\ 28.3 & 0.6 & 4.090 & 1998.679 & \text { 5-Sep-98 } & 6.9 \\ 34.8 & 1.0 & 5.088 & 1999.677 & \text { 4-Sep-99 } & 6.8 \\ 41.5 & 1.4 & 6.217 & 2000.806 & 21 \text {-Oct-00 } & 6.7\end{array}$


Hayward Fault USGS-SFSU Afterslip Site

$50.15 \mathrm{~km}$-Woodland Ave.

$\begin{array}{cccccc}\begin{array}{c}\text { movement } \\ (\mathrm{mm})\end{array} & \text { std. dev. } & \text { \# of years } & \text { year } & \begin{array}{c}\text { date } \\ \text { 27-Jan-70 }\end{array} & \begin{array}{c}\text { simple } \\ \text { average }\end{array} \\ 0.0 & & 0.000 & 1970.074 & \\ 104.6 & 2.6 & 22.792 & 1992.866 & \text { 12-Nov-92 } & 4.6 \\ 119.0 & 0.8 & 27.562 & 1997.636 & \text { 20-Aug-97 } & 4.3 \\ 125.0 & 1.0 & 28.605 & 1998.679 & \text { 5-Sep-98 } & 4.4 \\ 129.2 & 0.4 & 29.603 & 1999.677 & \text { 4-Sep-99 } & 4.4 \\ 141.1 & 2.7 & 30.732 & 2000.806 & \text { 21-Oct-00 } & 4.6\end{array}$


Hayward Fault USGS-SFSU Afterslip Site

$52.60 \mathrm{~km}$-Chapel of the Chimes Cemetery

$\begin{array}{cccccc}\begin{array}{c}\text { movement } \\ (\mathrm{mm})\end{array} & \text { std. dev. } & \text { \# of years } & \text { year } & \text { date } & \begin{array}{c}\text { simple } \\ \text { average }\end{array} \\ 0.0 & 1.3 & 0.000 & 1994.592 & \text { 4-Aug-94 } & \\ 5.9 & 2.1 & 1.052 & 1995.644 & \text { 23-Aug-95 } & 5.6 \\ 7.2 & 1.0 & 2.042 & 1996.634 & 19-\text { Aug-96 } & 3.5 \\ 14.6 & 0.9 & 3.041 & 1997.633 & 19-\text { Aug-97 } & 4.8 \\ 20.8 & 0.9 & 4.104 & 1998.696 & 11-\text { Sep-98 } & 5.1 \\ 26.1 & 0.9 & 5.104 & 1999.696 & 11-\text { Sep-99 } & 5.1 \\ 34.5 & 1.3 & 6.271 & 2000.863 & 11 \text {-Nov-00 } & 5.5\end{array}$


Hayward Fault USGS-SFSU Afterslip Site 59.09 km-Gilbert Ave.

$\begin{array}{cccccc}\begin{array}{c}\text { movement } \\ (\mathrm{mm})\end{array} & \text { std. dev. } & \text { \# of years } & \begin{array}{c}\text { year } \\ \text { date }\end{array} & \begin{array}{c}\text { simple } \\ \text { average }\end{array} \\ 0.0 & 0.5 & 0.000 & 1983.759 & \text { 4-Oct-83 } & \\ 17.4 & & 4.277 & 1988.036 & \text { 13-Jan-88 } & 4.1 \\ 28.7 & & 4.809 & 1988.568 & \text { 26-Jul-88 } & 6.0 \\ 28.7 & & 5.088 & 1988.847 & \text { 5-Nov-88 } & 5.6 \\ 27.5 & & 8.869 & 1992.628 & \text { 17-Aug-92 } & 3.1 \\ 30.6 & 0.9 & 9.126 & 1992.885 & \text { 19-Nov-92 } & 3.4 \\ 42.7 & 0.8 & 10.833 & 1994.592 & \text { 4-Aug-94 } & 3.9 \\ 54.3 & 0.4 & 11.885 & 1995.644 & \text { 23-Aug-95 } & 4.6 \\ 58.9 & 0.7 & 12.886 & 1996.645 & \text { 23-Aug-96 } & 4.6 \\ 77.4 & 0.4 & 13.885 & 1997.644 & \text { 23-Aug-97 } & 5.6 \\ 67.4 & 1.1 & 14.920 & 1998.679 & \text { 5-Sep-98 } & 4.5 \\ 76.2 & 0.5 & 15.920 & 1999.679 & \text { 5-Sep-99 } & 4.8 \\ 86.6 & 0.3 & 17.085 & 2000.844 & \text { 4-Nov-00 } & 5.1\end{array}$


Hayward Fault USGS-SFSU Afterslip Site 62.64 km-Hancock Ave.

$\begin{gathered}\text { movement } \\ (\mathrm{mm})\end{gathered}$
0.0
61.6
73.0
81.7
83.8
88.1
98.1

\begin{tabular}{|c|c|c|c|c|c|}
\hline std. dev. & \# of years & year & date & $\begin{array}{c}\mathrm{mm} / \mathrm{yr} \text { simple } \\
\text { average }\end{array}$ & $\begin{array}{c}\mathrm{mm} / \mathrm{yr} \text { simple } \\
\text { average after } \\
1994.627\end{array}$ \\
\hline & 0.000 & 1982.041 & 15-Jan-82 & & \\
\hline 1.8 & 12.586 & 1994.627 & 17-Aug-94 & 4.9 & \\
\hline 1.7 & 14.604 & 1996.645 & 23-Aug-96 & 5.0 & 5.4 \\
\hline 0.7 & 15.603 & 1997.644 & 23-Aug-97 & 5.2 & 6.4 \\
\hline 0.4 & 16.641 & 1998.682 & 6-Sep-98 & 5.0 & 5.5 \\
\hline 0.9 & 17.638 & 1999.679 & 5-Sep-99 & 5.0 & 5.2 \\
\hline 0.7 & 18.803 & 2000.844 & 4-Nov-00 & 5.2 & 5.9 \\
\hline
\end{tabular}


Hayward Fault USGS-SFSU Afterslip Site 63.10 km-Union St.

$\begin{array}{cccccc}\begin{array}{c}\text { movement } \\ (\mathrm{mm})\end{array} & \text { std. dev. } & \text { \# of years } & \text { year } & \text { date } & \begin{array}{c}\text { simple } \\ \text { average }\end{array} \\ 0.0 & 2.3 & 0.000 & 1993.019 & \text { 7-Jan-93 } & \\ 4.8 & 0.5 & 0.869 & 1993.888 & \text { 20-Nov-93 } & 5.5 \\ 9.4 & 2.0 & 1.573 & 1994.592 & \text { 4-Aug-94 } & 6.0 \\ 16.7 & 1.1 & 2.628 & 1995.647 & \text { 24-Aug-95 } & 6.4 \\ 20.7 & 0.9 & 3.131 & 1996.150 & \text { 24-Feb-96 } & 6.6 \\ 20.4 & 1.9 & 3.191 & 1996.210 & \text { 17-Mar-96 } & 6.4 \\ 20.3 & 2.2 & 3.629 & 1996.648 & \text { 24-Aug-96 } & 5.6 \\ 36.6 & 0.6 & 4.628 & 1997.647 & \text { 24-Aug-97 } & 7.9 \\ 42.5 & 1.7 & 5.663 & 1998.682 & \text { 6-Sep-98 } & 7.5 \\ 43.4 & 1.0 & 6.660 & 1999.679 & \text { 5-Sep-99 } & 6.5 \\ 52.8 & 0.8 & 7.825 & 2000.844 & \text { 4-Nov-00 } & 6.7\end{array}$


Hayward Fault USGS-SFSU Afterslip Site 65.29 km-Pine St.

\begin{tabular}{|c|c|c|c|c|c|c|}
\hline $\begin{array}{l}\text { movement } \\
\quad(\mathrm{mm})\end{array}$ & std. dev. & \# of years & year & date & $\begin{array}{c}\text { simple } \\
\text { average }\end{array}$ & $\begin{array}{c}\text { after } \\
1996.150\end{array}$ \\
\hline 0.0 & & 0.000 & 1989.216 & 20-Mar-89 & & \\
\hline 19.0 & 0.7 & 3.806 & 1993.022 & 8-Jan-93 & 5.0 & \\
\hline 19.2 & 0.8 & 4.672 & 1993.888 & 20-Nov-93 & 4.1 & \\
\hline 20.6 & 0.9 & 5.376 & 1994.592 & 4-Aug-94 & 3.8 & \\
\hline 25.4 & 0.4 & 6.436 & 1995.652 & 26-Aug-95 & 3.9 & \\
\hline 39.1 & 0.8 & 6.934 & 1996.150 & 24-Feb-96 & 5.6 & \\
\hline 40.9 & 2.0 & 6.975 & 1996.191 & 10-Mar-96 & 5.9 & \\
\hline 41.9 & 0.3 & 7.426 & 1996.642 & 22-Aug-96 & 5.6 & \\
\hline 44.9 & 0.5 & 8.431 & 1997.647 & 24-Aug-97 & 5.3 & 3.9 \\
\hline 45.1 & 1.2 & 9.466 & 1998.682 & 6-Sep-98 & 4.8 & 2.4 \\
\hline 49.4 & 0.5 & 10.480 & 1999.696 & 11-Sep-99 & 4.7 & 2.9 \\
\hline 58.6 & 0.8 & 11.647 & 2000.863 & 11-Nov-00 & 5.0 & 4.1 \\
\hline
\end{tabular}


Hayward Fault USGS-SFSU Afterslip Site 67.02 km-South Grimmer Blvd.

$\begin{array}{cccccc}\begin{array}{c}\text { movement } \\ (\mathrm{mm})\end{array} & \text { std. dev. } & \text { \# of years } & \begin{array}{c}\text { year } \\ \text { 0.000 }\end{array} & \begin{array}{c}\text { date } \\ \text { 1982.460 }\end{array} & \begin{array}{c}\text { 17-Jun-82 } \\ \text { average }\end{array} \\ 0.0 & & 10.978 & 1993.438 & \text { 9-Jun-93 } & 7.1 \\ 77.7 & 3.6 & 12.167 & 1994.627 & \text { 17-Aug-94 } & 6.3 \\ 76.2 & 2.6 & 13.690 & 1996.150 & \text { 24-Feb-96 } & 7.6 \\ 103.4 & 1.0 & 13.750 & 1996.210 & \text { 17-Mar-96 } & 7.7 \\ 105.8 & 1.1 & 14.188 & 1996.648 & \text { 24-Aug-96 } & 7.3 \\ 104.1 & 3.5 & 15.187 & 1997.647 & \text { 24-Aug-97 } & 7.0 \\ 106.7 & 1.1 & 16.225 & 1998.685 & \text { 7-Sep-98 } & 6.6 \\ 106.8 & 0.8 & 17.239 & 1999.699 & \text { 12-Sep-99 } & 6.4 \\ 109.7 & 3.4 & 18.403 & 2000.863 & \text { 11-Nov-00 } & 6.4 \\ 117.8 & 3.0 & & & & \end{array}$


Hayward Fault USGS-SFSU Afterslip Site $67.21 \mathrm{~km}$-Onondaga Dr.

$\begin{array}{ccccccc}\begin{array}{c}\text { movement } \\ (\mathrm{mm})\end{array} & \text { std. dev. } & \begin{array}{c}\text { \# of years } \\ 0.000\end{array} & \begin{array}{c}\text { year } \\ 1982.400\end{array} & \begin{array}{c}\text { date } \\ \text { 26-May-82 }\end{array} & \begin{array}{c}\text { simple } \\ \text { average }\end{array} & 1994.627 \\ 0.0 & & 12.227 & 1994.627 & \text { 17-Aug-94 } & 3.4 & \\ 41.5 & 0.9 & 14.245 & 1996.645 & 23 \text {-Aug-96 } & 2.8 & -0.8 \\ 39.9 & 2.3 & 15.244 & 1997.644 & 23-\text { Aug-97 } & 2.8 & 0.2 \\ 42.1 & 0.5 & 16.285 & 1998.685 & 7-\text {-Sep-98 } & 2.5 & -0.1 \\ 41.2 & 0.6 & 17.299 & 1999.699 & 12-\text { Sep-99 } & 2.4 & -0.1 \\ 41.2 & 0.6 & 18.463 & 2000.863 & 11 \text {-Nov-00 } & 2.3 & 0.0 \\ 41.7 & 1.5 & 18.4 & & & & \end{array}$




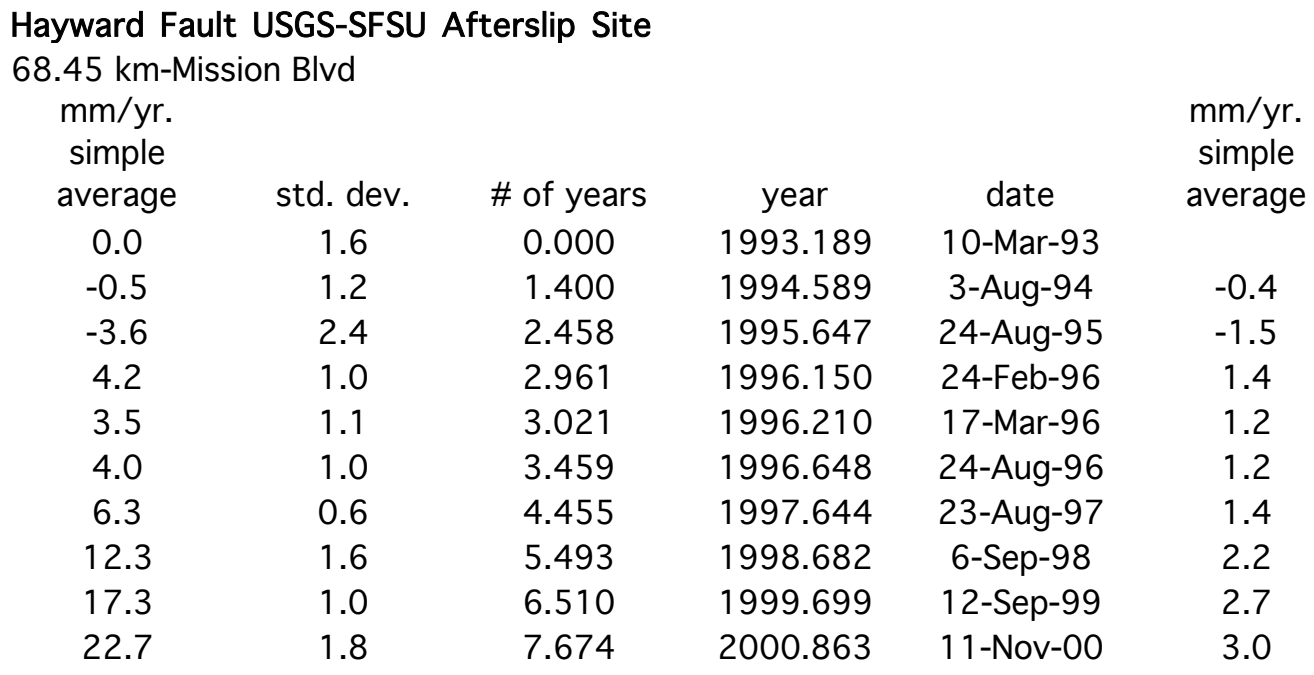

